Foundations and Trends ${ }^{\circledR}$ in

Computer Graphics and Vision

Vol. 9, No. 3-4 (2013) 149-254

(C) 2015 J. Ackermann and M. Goesele

DOI: $10.1561 / 0600000065$

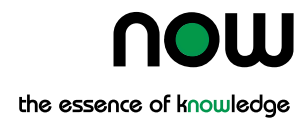

\title{
A Survey of Photometric Stereo Techniques
}

\author{
Jens Ackermann \\ TU Darmstadt \\ jens.ackermann@gris.informatik.tu-darmstadt.de \\ Michael Goesele \\ TU Darmstadt \\ michael.goesele@gris.informatik.tu-darmstadt.de
}




\section{Contents}

$\begin{array}{lll}1 & \text { Introduction } & 151\end{array}$

1.1 Scope of this survey . . . . . . . . . . . . . . . . . 152

1.2 Overview . . . . . . . . . . . . . . . . . . . 153

$\begin{array}{lll}2 & \text { Background } & 154\end{array}$

$2.1 \quad$ Light-Matter-Interaction . . . . . . . . . . . . . . . . . . . 154

2.2 Lambertian photometric stereo . . . . . . . . . . . . 155

2.3 Error sources . . . . . . . . . . . . . . . . . . . . . . . . . . . 156

2.4 Baseline quality . . . . . . . . . . . . . . . . . . . . . . . 164

2.5 Integration of surface gradients . . . . . . . . . . . . 167

3 Foundations 171

4 Unknown lighting 176

4.1 Point light source . . . . . . . . . . . . . . . . . . . 177

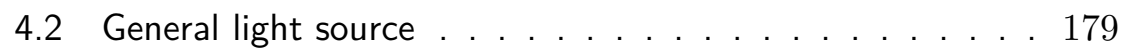

4.3 Other generalizations . . . . . . . . . . . . . . . . . . . . . . . 181

5 Unknown reflectance 183

5.1 Diffuse and specular components . . . . . . . . . . . . . 184

5.2 More complex BRDF models . . . . . . . . . . . . . . 186

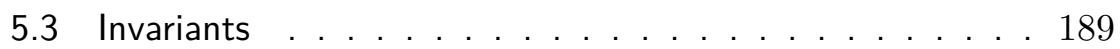


$\begin{array}{lll}6 & \text { Outlier handling } & 194\end{array}$

6.1 Explicit treatment . . . . . . . . . . . . . . . . . . . . . 195

6.2 General deviations . . . . . . . . . . . . . . . . . . . . 197

7 Unknown lighting and reflectance 200

7.1 Diffuse specular separation . . . . . . . . . . . . . . . . . 201

7.2 Parametric models . . . . . . . . . . . . . . . . . . 202

7.3 Appearance profiles . . . . . . . . . . . . . . . . . 202

8 Multi-view settings 206

8.1 Fixed illumination . . . . . . . . . . . . . . . . . . 207

8.2 Differential motion. . . . . . . . . . . . . . . . . . . . 208

8.3 Wide baseline . . . . . . . . . . . . . . . . . . . . . . . . . 210

9 Outside the lab 218

9.1 Internet data . . . . . . . . . . . . . . . . . . . . . . . . . . 219

9.2 Natural illumination . . . . . . . . . . . . . . . . . . . 224

10 Conclusion 228

10.1 Summary . . . . . . . . . . . . . . . . . . . . . . . . . . 228

10.2 Discussion and outlook . . . . . . . . . . . . . . 231

10.3 Looking ahead . . . . . . . . . . . . . . . . . . . . . . 233

\begin{tabular}{ll}
\hline Acknowledgements & 235
\end{tabular}

References 236 


\section{Abstract}

Reconstructing the shape of an object from images is an important problem in computer vision that has led to a variety of solution strategies. This survey covers photometric stereo, i.e., techniques that exploit the observed intensity variations caused by illumination changes to recover the orientation of the surface. In the most basic setting, a diffuse surface is illuminated from at least three directions and captured with a static camera. Under some conditions, this allows to recover per-pixel surface normals. Modern approaches generalize photometric stereo in various ways, e.g., relaxing constraints on lighting, surface reflectance and camera placement or creating different types of local surface estimates.

Starting with an introduction for readers unfamiliar with the subject, we discuss the foundations of this field of research. We then summarize important trends and developments that emerged in the last three decades. We put a focus on approaches with the potential to be applied in a broad range of scenarios. This implies, e.g., simple capture setups, relaxed model assumptions, and increased robustness requirements. The goal of this review is to provide an overview of the diverse concepts and ideas on the way towards more general techniques than traditional photometric stereo.

J. Ackermann and M. Goesele. A Survey of Photometric Stereo Techniques. Foundations and Trends ${ }^{\circledR}$ in Computer Graphics and Vision, vol. 9, no. 3-4, pp. 149-254, 2013.

DOI: $10.1561 / 0600000065$. 


\section{Notation}

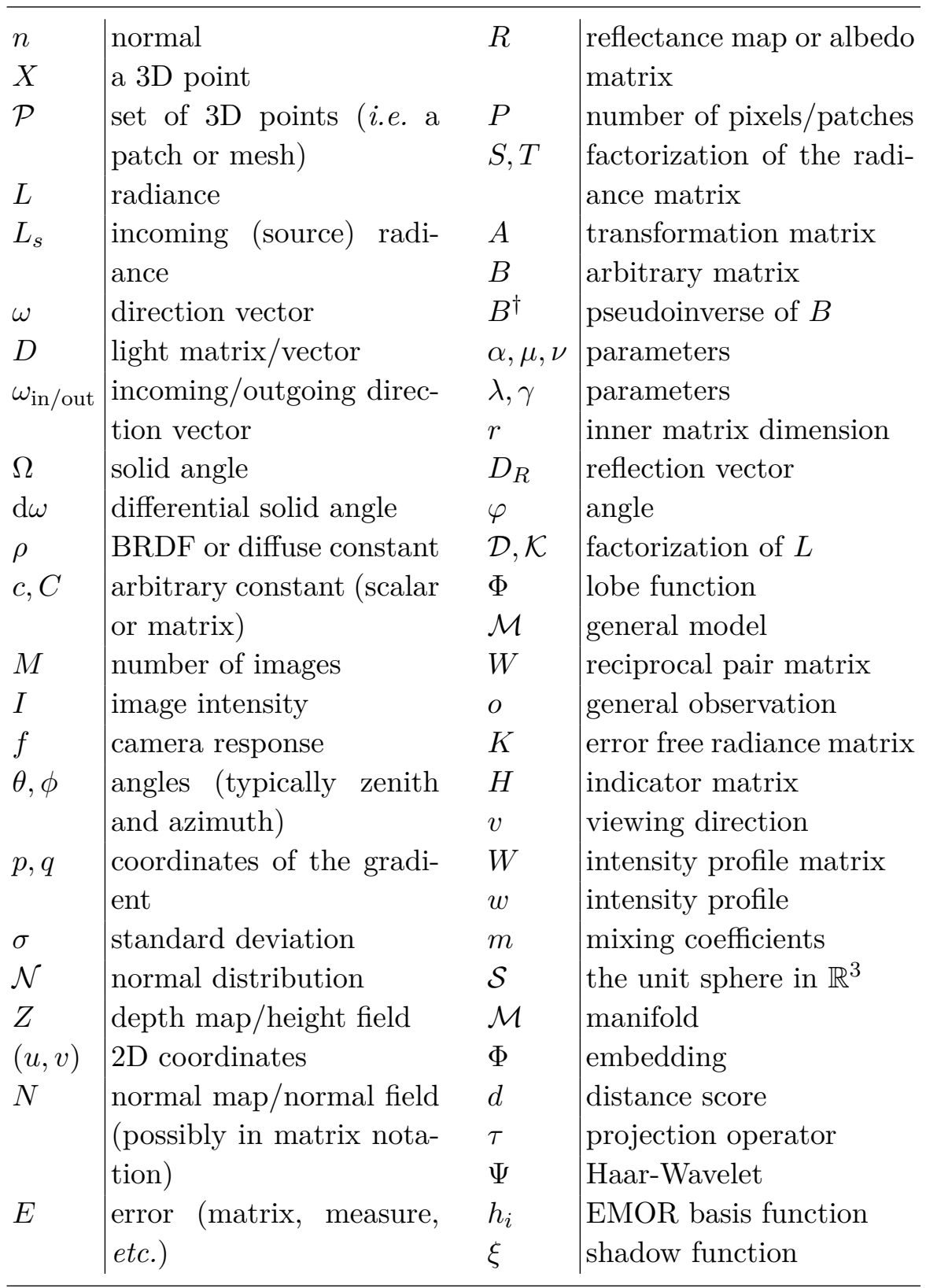


The shape of an object, its reflectance, and the incoming illumination define the image that the object forms in our eyes or on a camera sensor. Even for a uniformly colored, diffuse object, the shading typically changes depending on the local orientation of the surface and the properties of the incoming light. Highlights form on glossy or specular surfaces, providing additional cues about the surface shape and reflectance as well as the illumination. Humans are remarkably good at deducing such information from images even under very general conditions (Thompson et al. [2011]). In contrast, recovering one or several of these individual components is a much more difficult task for a computer system.

To solve such inverse problems, different techniques exist such as shape from defocus (Pentland 1987), shape from texture (Blostein and Ahuja [1989]), or (multi-view) stereo (Seitz et al. [2006]). In this survey, we direct our attention to photometric approaches. These exploit the intensity variations caused by illumination changes. Typically, the input is a set of images of an object or scene under a varying set of lighting conditions, and the output is the 3D shape, e.g. encoded as a field of surface normals. 
The goal of classic photometric stereo is to recover the surface orientation from a known combination of reflectance and lighting in multiple images. Starting from this, a rich body of literature was developed that generalizes the problem and its solution in various directions. Many of these approaches assume a controlled capture setup because wellbehaved input data is crucial for inverse problems. These requirements constrain the application mostly to the research community and production settings. In this survey, we put a focus on approaches with a potential to broaden this applicability, e.g., through simple capture setups or relaxed requirements.

\subsection{Scope of this survey}

Shape and appearance reconstruction have connections to different fields in computer vision, computer graphics, optimization theory, statistics, optics, etc. We will only cover those areas that are most relevant for this survey. These are approaches that rely on varying illumination in multiple images to recover at least the surface orientation and possibly even reflectance and illumination.

That excludes for example the large area of shape from shading approaches that operate on single images (Zhang et al. [1999, Johnson and Adelson 2011], Oxholm and Nishino [2012, Han et al. 2013]) and the related works on intrinsic image decomposition (e.g. Barron and Malik 2012, 2013]). These are highly ill-posed problems and their solution requires strong regularization. Using multiple images provides more information and better constrains the result space. Some works (e.g. Magda et al. [2001, Koppal and Narasimhan [2007], Liao et al. [2007]) exploit the fall-off in radiance with the distance from a near point light or more general changes in the apparent source intensity (e.g. Davis et al. 2005). Our focus will be on illumination variations caused by directional changes in the incident light. We also do not consider purely specular surfaces (Healey and Binford 1986, Bonfort and Sturm [2003], Tarini et al. [2005], Chen et al. [2006], Nehab et al. [2008], Weinmann et al. [2013]) or the specialized approaches for face (Debevec et al. [2000], Zhou et al. [2007], Ghosh et al. [2011]) and dy- 
namic performance (Ahmed et al. [2008], Wlasic et al. [2009], Wu et al. [2012] ) capturing. Similarly, we will mention only a few approaches that require highly complex capture setups because they have less potential to be adapted for unconstrained environments

In many modern works ideas from different disciplines get combined, e.g. when silhouette constraints are merged with photometric cues in certain multi-view approaches. This makes a clear distinction to other fields difficult at times. We include such cases in our discussion if it suits the overall perspective.

\subsection{Overview}

We begin this survey with an introduction for readers unfamiliar with photometric stereo methods in Chapter 2. It serves as a tutorial and to promote awareness for the challenges present in practical applications of such techniques. Before proceeding to the main part, we briefly discuss some early works in Chapter 3 that laid the foundations for present-day research.

Finding an optimal categorization of photometric approaches to structure this survey is not trivial. We decide for a selection scheme based on common challenges among the algorithms such as unknown lighting in Chapter 4, complex reflectance in Chapter 5, or extremely uncontrolled conditions in Chapter 9. Within each category we aim at presenting relevant representatives instead of cluttering the exposition with lists of similar approaches.

Finally, we conclude the survey in Chapter 10 with a summary of the most important concepts and our views on future developments. 


\section{2}

\section{Background}

This chapter summarizes some theoretical and practical background information to make the survey more self-contained. It aims to be an introduction for readers unfamiliar with photometric reconstruction methods. In $\$ 2.1$ and $\$ 2.2$, we establish some notation that is used throughout this work and introduce a few formulas that will be referenced later. We then explain the challenges arising in real-world setups and discuss about the accuracy to be expected from photometric stereo in $\$ 2.3$ and $\$ 2.4$. The results obtained from such techniques are typically estimates of the surface gradient or orientation. If the actual $3 \mathrm{D}$ geometry, e.g. represented as a height map, is required, such a normal field has to be integrated. $\$ 2.5$ presents common strategies towards that end.

\subsection{Light-Matter-Interaction}

When light interacts with matter on a macroscopic level, i.e. discounting wave-like effects, it can be absorbed, transmitted, or reflected. Although all of these effects occur simultaneously, we will here only consider the fraction of light that is reflected. 
Let $X \in \mathbb{R}^{3}$ be a surface point with normal $n \in \mathcal{S}:=\{Y \in$ $\left.\mathbb{R}^{3},\|Y\|=1\right\}$. The solid angle of an object with respect to a point $X$ is the surface area of its projection onto a unit sphere around $X$. Let $L_{s}\left(\omega_{\text {in }}\right)$ be the incoming radiance from an infinitesimal solid angle $d \omega_{\text {in }}$ centered around the direction $\omega_{\text {in }} \in \mathcal{S}$. The irradiance on a small patch around $\mathcal{P}$ coming from the whole hemisphere of directions $\Omega$ is the integral

$$
\int_{\Omega} L_{s}\left(\omega_{\text {in }}\right)\left\langle n, \omega_{\text {in }}\right\rangle \mathrm{d} \omega_{\text {in }} .
$$

The total radiance $L\left(\omega_{\text {out }}\right)$ leaving $X$ in the direction $\omega_{\text {out }}$ is then given as

$$
L\left(\omega_{\text {out }}\right)=\int_{\Omega} \rho\left(\omega_{\text {out }}, \omega_{\text {in }}\right) L_{s}\left(\omega_{\text {in }}\right)\left\langle n, \omega_{\text {in }}\right\rangle \mathrm{d} \omega_{\text {in }},
$$

where the function $\rho$ is the proportionality factor between the radiance leaving the surface point and the irradiance caused by incoming light from $\omega_{\text {in }}$. Nicodemus et al. [1977] calls $\rho$ the bidirectional reflectancedistribution function (BRDF). A detailed look into these radiometric topics is provided, e.g., in Chapter 4 of Forsyth and Ponce 2011.

\subsection{Lambertian photometric stereo}

Many photometric stereo techniques, $c f . \$ 4.1$, consider the special case of Lambertian reflectance, i.e. $\rho\left(\omega_{\text {out }}, \omega_{\text {in }}\right)=\rho / \pi$, and a single point light source $L_{s}\left(\omega_{\text {in }}\right)=c \delta\left(\omega_{\text {in }}-\omega_{s}\right)$ from direction $\omega_{s}$. In this case, the outgoing radiance is independent of the direction $\omega_{\text {out }}$ and the integral in Equation 2.2 vanishes:

$$
L=\frac{\rho c}{\pi} \max \left(\left\langle n, \omega_{\mathrm{in}}\right\rangle, 0\right) .
$$

The clamping of negative dot products will be implicitly assumed from here on.

Observing a Lambertian surface point $M$ times under varying light directions $\omega_{\text {in }}$, which we denote as direction vectors $D_{1}, \ldots, D_{M} \in \mathbb{R}^{3}$, and source strength $c_{1}, \ldots, c_{M}$ yields radiance values

$$
L=\left(L_{1}, \ldots, L_{M}\right)^{T}=\frac{\rho}{\pi}\left(n^{T} D_{1} c_{1}, \ldots, n^{T} D_{M} c_{M}\right)^{T}=: \frac{\rho}{\pi} D \cdot n,
$$

where the scaled row vectors $c_{i} D_{i}$ form the $M \times 3$ light matrix $D$. 
Expressing the Lambertian image formation using matrix notation makes all tools from linear algebra available to study the photometric stereo problem. For $M \geq 3$ and three linearly independent directions, the linear system is overdetermined but has full rank. Thus, $D^{T} D$ is invertible and the pseudoinverse $\left(D^{T} D\right)^{-1} D^{T}$ exists. Multiplying Equation 2.4 from the left

$$
\left(D^{T} D\right)^{-1} D^{T} L=\frac{\rho}{\pi}\left(D^{T} D\right)^{-1} D^{T} D \cdot n=\frac{\rho}{\pi} n
$$

allows to compute the normal from $D$ and $L$ :

$$
n=\frac{\left(D^{T} D\right)^{-1} D^{T} L}{\left\|\left(D^{T} D\right)^{-1} D^{T} L\right\|}
$$

\subsection{Error sources}

In a real setup, photometric stereo techniques have to deal with various sources of error. We believe that these need to be understood to achieve the goal of broader applicability and dedicate this section to the discussion of the most common ones. In addition, a couple of simple experiments will provide an intuition about the impact of some of the errors.

\subsubsection{Noise}

All photometric techniques rely on radiance measurements. Like with any other measurement process, the observed values might be subject to noise. One can reduce its impact by capturing additional data, e.g. averaging multiple images under the same conditions. Whether its influence can be neglected, however, depends on the specific application and setting.

Sometimes, the noise levels for specialized equipment like cameras for industrial inspection or microscopy are provided in their specification sheet, cf. Point Grey Research, Inc. [2015]. For consumer cameras, however, such data is rarely available. Nevertheless, these cameras are regularly used in computer vision applications and will play a central role in bringing photometric methods to a broader audience. Quantifying sensor noise precisely requires highly controlled conditions, 


\begin{tabular}{lcccccc}
\hline & \multicolumn{3}{c}{ planar } & & \multicolumn{2}{c}{ sphere } \\
\cline { 2 - 4 } \cline { 7 - 8 } & $1 / 20 \mathrm{~s}$ & $1 / 10 \mathrm{~s}$ & $1 / 8 \mathrm{~s}$ & & dark & bright \\
\hline mean (\%) & 27.8 & 56.7 & 71.6 & & 28.4 & 48.2 \\
deviation (\%) & 0.5 & 0.8 & 1.0 & & 0.6 & 0.8 \\
\hline
\end{tabular}

Table 2.1: Statistics computed over a small patch in the green channel of 51 images from an 18 megapixel consumer sensor (Canon EOS 700D). The experiment for the planar target is repeated for three different exposure times and a patch of $16 \times 16$ pixel. For the sphere, the exposure time is $1 / 10$ s and the patch size is $10 \times 10$ pixel. Results are listed in percent of the full sensor range.

$c f$. European Machine Vision Association [2010]. Instead, we ask what the noise levels are in an experimental setup (in situ) with a consumer camera in a darkened room containing a high-quality, but not exceptional light source and a real object.

Noise levels depends very much on the sensor model, as shown by the data in Point Grey Research, Inc. 2015. Table 2.1 gives an impression of the noise behavior for the Canon EOS 700D. For exposure times of $1 / 20 \mathrm{~s}, 1 / 10 \mathrm{~s}, 1 / 8 \mathrm{~s}$, we repeatedly captured 51 raw images of a planar reflectance standard under constant illumination. The left part of Table 2.1 shows the statistics computed in the green channel of a small patch in that image stack. We notice a clear dependence of the standard deviation on the mean intensity.

To exclude effects of exposure time changes, we keep it fixed at $1 / 10$ s and perform a second experiment with a diffuse sphere. Statistics are now computed over two patches corresponding to dark and bright regions on the sphere. The right part of Table 2.1 shows a similar relationship of mean and standard deviation. This indicates that it does not depend on the exposure time. Overall, we observe that in reasonably exposed images the noise level is below $1 \%$ of the full sensor range.

\subsubsection{Shadows}

The argumentation in $\$ 2.2$ leading to the solution of the photometric stereo problem in Equation 2.6 implicitly assumes that the surface is 
not in shadow in any of the images. If something blocks the light, the observed radiance will vanish independent of the orientation of the surface. Thus, Equation 2.6 is not fulfilled and any solution based on that model will be incorrect. Similar restrictions also apply for more advanced lighting and reflection models than the Lambertian one.

As we will see in $\$ 6.1$, shadows still convey some information about the scene that can be exploited. In general, however, it is important to either avoid shadows already during capture. One solution is, e.g., to place the light source close to the camera. This can lead, however, to illconditioned inverse problems if the lights become too similar. Another strategy is to detect and address shadows after capture, e.g. excluding those observations from further computations.

\subsubsection{Interreflections}

The exitant radiance at a point according to Equation 2.2 depends on the incoming radiance from all directions. The integral vanishes in Equation 2.3 for a single point light source. This is, however, only strictly valid for an isolated surface patch in empty space or convex objects in general. In all other cases, the incoming radiance at a point $p$ will consist of the radiance exiting the light source and the radiance leaving any other surface point in the direction towards $p$. These global illumination effects are usually not modeled in the kind of inverse problems studied here. Whether this leads to significant errors depends on the scene geometry and reflectance, e.g. a mirror reflecting light onto $p$. In practice, at least the effects of interreflections from the environment can be reduced by covering non-target surfaces with black cloth. This is, however, not easily possible in uncontrolled scenarios.

\subsubsection{Camera}

As discussed above, noise is present in any measurement process. In the case of modern photometric techniques, the measurement device is a camera and as such has some additional sources of systematic error. 


\section{Falloff}

One kind of error is introduced by the optical system of the camera. So called vignetting leads to reduced image intensities in peripheral regions compared to the center. One way to address this issue is by calibrating the falloff based on an evenly illuminated gray card. Another possibility is to reconstruct only in small regions - in terms of the image planewhere the falloff can be assumed constant.

\section{Radiance to pixel conversion}

The camera transforms continuous radiance $L$ into digital pixel values I. Most cameras use a sensor with an added color filter array. Thus, individual sensor elements capture contributions either of the red, green, or blue channel. These contributions have to be interpolated over the image plane to produce a full-sized, colored image with RGB information at each pixel. Effects introduced by this interpolation are relatively small compared to other errors (and can often be avoided by using raw image data).

Discounting any color space conversions or image processing such as sharpening filters, a simple model of the transformation from radiance to pixel intensity is given by the camera response curve $f(L):=I$. If raw values are available, the response is usually a clamped, linear function

$$
f(L)=\min \left(\max \left(\alpha L-I_{\text {dark }}, 0\right), I_{\text {sat }}\right)
$$

for a certain scaling $\alpha$ that depends on the sensor and the quantization resolution (ranging from 10 to 14 bit for typical consumer cameras). $I_{\text {dark }}$ accounts for the nonzero output level that is present even if no light actually reaches the camera, e.g. with the lens cap attached.

If raw values are not available, the response of the camera is nonlinear and has to be calibrated. Lots of methods for this task exist, e.g. based on calibration targets (Grossberg and Nayar. 2004]), stacks of differently exposed images (Debevec and Malik [1997, Robertson et al. [1999, 2003], Kim et al. 2008]), color edges in a single image (Lin et al. [2004]), or probabilistic color de-rendering (Xiong et al. [2012]). 
Ignoring non-linear responses is almost never a viable option since it can lead to drastically different results. We illustrate this with a short example for a simplified setting. Let $n=(0,0,1)$ and assume that the light forms an angle $\theta \in] 0, \pi / 2[$ with the z-axis in two images and shines from above in the third:

$$
D=\left(\begin{array}{ccc}
\sin \theta & 0 & \cos \theta \\
0 & \sin \theta & \cos \theta \\
0 & 0 & 1
\end{array}\right), \quad D^{-1}=\left(\begin{array}{ccc}
\frac{1}{\sin \theta} & 0 & -\frac{\cos \theta}{\sin \theta} \\
0 & \frac{1}{\sin \theta} & -\frac{\cos \theta}{\sin \theta} \\
0 & 0 & 1
\end{array}\right)
$$

The ensuing radiance values are

$$
L=\left(L_{1}, L_{2}, L_{3}\right)=(\cos \theta, \cos \theta, 1) .
$$

Applying photometric stereo as in $\$ 2.2$ on the non-linear pixel intensities $I=f(L)$ yields a normal $\tilde{n}$ which deviates from the true normal $n$ by an angle of

$$
\operatorname{acos}(\langle\tilde{n}, n\rangle)=\operatorname{acos}\left(\frac{\left\langle D^{-1} f(L), n\right\rangle}{\left\|D^{-1} f(L)\right\|}\right)=\operatorname{acos}\left(\frac{f(1)}{\left\|D^{-1} f(L)\right\|}\right) .
$$

Figure 2.1 shows the angular error for varying $\theta$ and $\gamma$ in the commonly assumed - though often oversimplifying - gamma curve response model

$f(x)=x^{\gamma}$. The systematic deviations from the true normal illustrate the importance of applying the correct inverse response prior to any photometric reconstruction.

\section{Camera motion}

Accurate correspondences between pixels in all images are important for photometric stereo. If no image alignment is applied as preprocessing, the use of a solid tripod and remote trigger are essential. The impact of this error depends on the scene content, e.g. it would not matter for a homogenous plane.

In multi-view settings such as discussed in Chapter 8 the camera is moved on purpose and its position is often assumed to be known. If this knowledge is inaccurate, e.g. due to bad pose estimation, the projection of a $3 \mathrm{D}$ point will occur at the wrong pixel, thus breaking correspondence. Combining pixels from multiple images will then mix information from different surface points leading to erroneous results. 

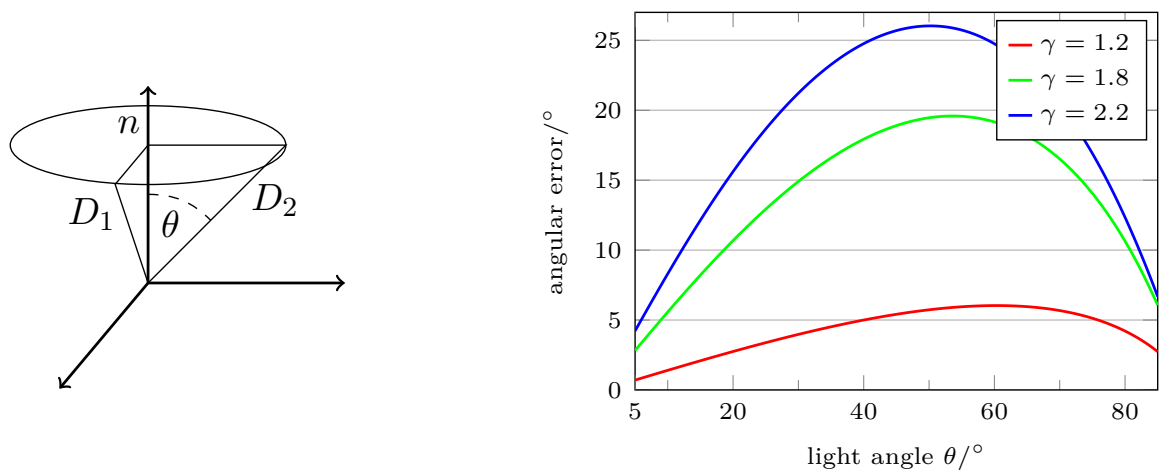

Figure 2.1: Impact of the non-linear response on photometric stereo. Left: Example setup with two light directions at an angle $\theta$ with the normal and the third shining from above $\left(D_{3}=-n\right)$. Right: The angular deviation of the reconstructed normal if the intensities are not linearized properly.

\subsubsection{Light source}

For those algorithms relying on calibrated light sources, their position and intensity become another set of observables that are again subject to noise. Many ways to measure these have been proposed. The approaches differ in accuracy, capture setup, lighting model, additional constraints, and in the intended application, e.g., renderings in augmented reality, shape reconstruction, or image-based relighting. For photometric stereo applications, it is often assumed that the light is a simple point light source and that its location is known very precisely.

Some works exploit cast shadows (Panagopoulos et al. [2011]), sample the complete incoming light field (Sato et al. [1999, Kanbara and Yokoya [2004]), or estimate the light source from stationary images (Winnemoeller et al. [2005]). Approaches that minimize an intensity error compare actual images of a scene with known geometry and reflectance against renderings obtained from the current light estimate (Hara et al. 2005, Weber and Cipolla [2001, Xu and Wallace [2008]).

These are, however, unsuited in contexts where the shape is to be reconstructed in a later step.

We narrow down the discussion to the estimation of point light sources based on target objects of known, usually spherical, geometry. 
If space allows to place the light far away from the scene, techniques to recover its overall direction have been proposed by Dosselmann and Yang [2013], Wang and Samaras 2002, Wong et al. [2008], or Zhou and Kambhamettu 2002. If the light cannot be placed sufficiently far away, however, its direction is not constant for every scene point, and the irradiance falls off with the square of the distance. Masselus et al. [2002 and Powell et al. [2001] demonstrate that some of the ideas used for recovering infinitely distant illumination can be readily used to estimate positions of near point light sources if applied to multiple spheres. The framework of Zhou and Kambhamettu 2004 is even able to triangulate an area light source by placing a specular sphere at different locations in the scene.

Here, we assume a single point light source and perform a small experiment to asses the influence of the camera on reconstructions obtained from a mirroring sphere. We also try to give a rough idea of the errors that one can expect in such a standard lab setting. For this experiment, we keep the light fixed at approximately $4 \mathrm{~m}$ distance and move a reflective sphere to 20 different positions spread about $25 \mathrm{~cm}$ around the center of the scene. Each sphere leads to a highlight that is observed by the camera (in our case a Canon 700D with a $110 \mathrm{~mm}$ lens). Given the radius and the position of the spheres, we can compute the light direction for each of the highlights by shooting a ray towards the sphere and reflecting it around the normal at the intersection point. Taking the perspective projection of the lens into account, the rays through the highlight pixels will fan out slightly. If we instead make the simplifying assumption of a purely orthographic camera - an assumption applied by many photometric stereo methods - these rays will all be parallel.

To compare both models, we create a synthetic dataset that allows us to study them without noise or inaccuracies in the highlight detections or sphere positions. For a point light source and a perspective camera, we analytically determine the highlight pixels and the center of the sphere in the image. We adjusted the position of the light source (sitting behind, above, and to the left of the camera) to match the highlights of the real experiment as closely as possible. We then reconstruct 

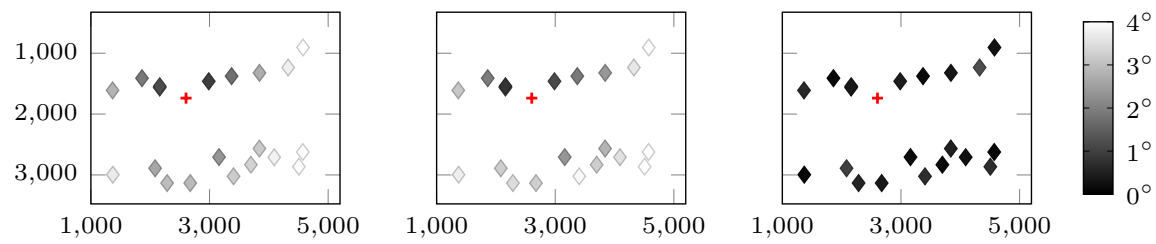

Figure 2.2: Accuracy of light direction estimation from a mirrored sphere. Each marker is the projection of one of the 20 sphere positions onto the image plane ( $\mathrm{x}$ and $y$-axes are in pixels). The gray value encodes the angular deviation from the synthetic ground truth. A cross indicates the optical axis of the camera. The varying distance of the sphere positions from the camera is not shown in this plot. Light directions reconstructed based on a simplifying orthographic camera model show a strong spatial dependency, both in a simulated, noise-free (left) and a real experiment (middle). For a perspective reconstruction, the errors between experiment and synthetic ground truth are all below $1.2^{\circ}$ (right).
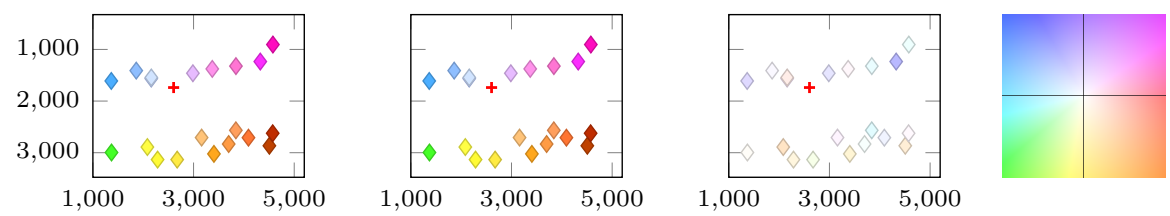

Figure 2.3: Visualizing the direction of the error for the same experiments as in Figure 2.2. For each sphere, the difference of the stereographic projections of the reconstructed light direction and the ground truth are color mapped according to the reference on the right.

the light direction for each sphere assuming an orthographic model. Figure 2.2 shows the angular deviation from the directions computed from the true perspective model. The direction of the error is visualized in Figure 2.3. As expected the error increases towards the edges of the field of view where the orthographic model deviates more strongly from a perspective one.

Next, we evaluate the gap between theory and practice by comparing the synthetic light directions with those reconstructed from the real experiment. For the perspective reconstruction, the deviation is below $1.2^{\circ}$ in all cases and does not show strong spatial variations in Figure 2.2. This indicates that effects from an inaccurate calibration or noisy highlight detections cause only minor errors. The same holds for 
the orthographic reconstruction which behaves similar to the simulated case.

Nevertheless, the performance could be improved by taking erroneous detections into account. Aoto et al. 2012 and Ackermann et al. 2013 allow for such inaccuracies by considering the reprojection error in the triangulation of near light sources.

\subsection{Baseline quality}

Given the large number of potential error sources discussed in $\$ 2.3$. one might ask whether photometric techniques have any chance of success. Common quality measures for the overall result of such methods are the angular normal error, the height differences of the integrated surface, or the intensity error in rendered images. Here, we use the angular error and conduct a controlled experiment in order to provide an intuition of the quality level that can be achieved. The controlled setting thereby establishes a baseline for more complex algorithms that will be discussed later.

\section{Experiment}

The target object is a sphere made of Spectralon, a highly Lambertian material. We use a sharply defined light source with a very stable radiance output that we place several meters away to approximate a distant point light. To estimate the light direction, we place two mirror spheres in the scene. Since the extent of the whole setup is small, the respective light vectors are very similar and we just average them. The experiment is performed with a consumer camera (Canon EOS 700D) equipped with a $135 \mathrm{~mm}$ telephoto lens. We took great care to avoid the error sources listed in $\$ 2.3$ as much as possible, e.g. covering all surfaces with cloth, using raw images, averaging ten pictures for each light position to reduce noise, etc.

Figure 2.4 contains the results for all pixels that were never in shadow for any of the six light sources. The error in Figure 2.5 is spatially varying because the real world surface has fine ridges, porous structure, and several pronounced dents that deviate from a true 

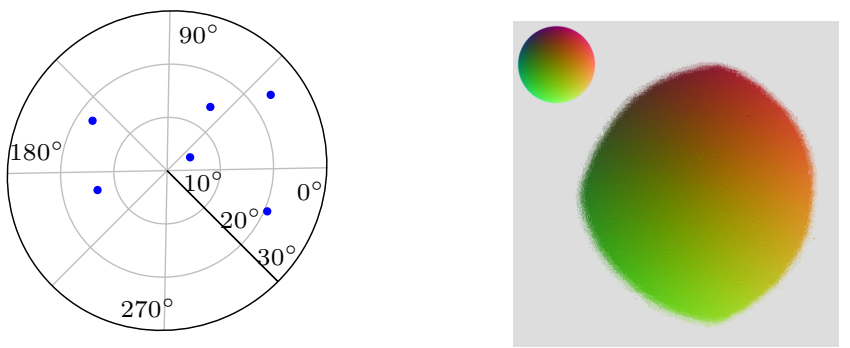

Figure 2.4: Experimental results for all pixels that were never in shadow for any of the six light sources. Left: The estimated light source directions in spherical camera coordinates. Right: Color coded normals of the reconstruction and an ideal sphere (inset).
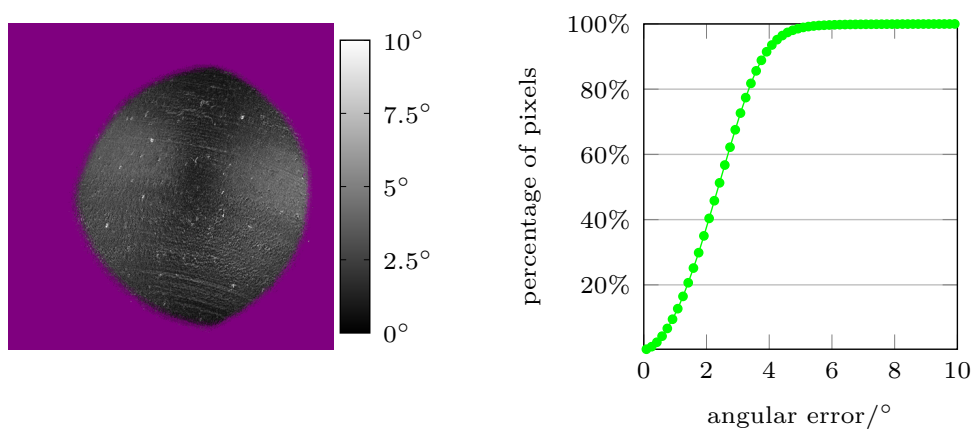

Figure 2.5: Comparison with an ideal sphere. Left: The spatial error distribution (mean: $2.5^{\circ}$, variance: $1.3^{\circ}$ ) shows fine ridges and dents where the target object deviates from an ideal sphere. Right: A cumulative histogram of the angular errors corresponding to the reconstruction on the left.

sphere. In addition, we observe a low-frequency bias. The errors are higher on the left and right side than on the top or bottom. One possible source for this systematic error is the deviation of the Spectralon BRDF from a true Lambertian reflector as pointed out by Bhandari et al. 2011]. Another lies in the configuration of light sources which yields a non-uniform error distribution. The effect of the latter will be confirmed through synthetic experiments below, $c f$. Figure 2.6

Overall, the histogram in Figure 2.5 shows that the deviation from ground truth is about $2.5^{\circ}$ in general. These findings are in line with other experiments on photometric stereo with known light sources as 


\begin{tabular}{|c|c|c|c|}
\hline Work & Technique & Comparison & Error \\
\hline Ray et al. 1983. & $\mathrm{C}$ & sphere & $<5^{\circ}$ \\
\hline Tagare and deFigueiredo 1991 & $\mathrm{C}$ & sphere & $4.8^{\circ}$ \\
\hline Wu and Tan 2013 & $\mathrm{C}+\mathrm{U}$ & sphere & $4^{\circ}$ \\
\hline \begin{tabular}{l|l|l|} 
Shi et al. $[2010$ \\
\end{tabular} & $\mathrm{U}$ & calibrated PS & $6-7^{\circ}$ \\
\hline Favaro and Papadhimitri 2012 & $\mathrm{U}$ & calibrated PS & $5-12^{\circ}$ \\
\hline Papadhimitri and Favaro 2013 & $\mathrm{U}$ & calibrated PS & $2-3^{\circ}$ \\
\hline \begin{tabular}{l|l|l} 
Abrams et al. 2012 \\
\end{tabular} & S & Google Earth & $20^{\circ}$ \\
\hline Tunwattanapong et al. 2013 & $\mathrm{~S}$ & sphere & $>5^{\circ}$ \\
\hline
\end{tabular}

Table 2.2: Evaluation results in a selection of works on calibrated (C) and uncalibrated (U) photometric stereo. Also listed are two examples of specialized techniques (S) with more complex models. The comparisons are either performed with another, presumably more accurate, technique as reference or based on objects with known geometry such as a sphere or simple models from Google Earth.

listed in Table 2.2. We conclude that in a controlled setting calibrated photometric stereo achieves an accuracy of about $3^{\circ}-5^{\circ}$. The table also indicates that uncalibrated techniques differ from calibrated ones by several degrees. If compared to ground truth geometry, however, they achieve similar results as shown by Wu and Tan 2013. Obviously, the less assumptions about the target, e.g. regarding reflectance, or the capture setup are made, the bigger these errors may become as shown by two exemplary techniques in the bottom rows.

\section{Error Analysis}

The evaluations above and in Table 2.2 are based on empirical data. We can also take an ab initio point of view to study the effect of the measurement errors. Based on the assumption of known error magnitudes in the image intensity and light measurements, Ray et al. 1983 present a theoretical error analysis for the Lambertian case. They assume a setup with three light sources on a circle around the optical axis. Then, the photometric stereo problem can be solved in closed form, cf. Equation 2.6. We encode this in a function $h$ :

$$
n=h\left(I_{1}, I_{2}, I_{3}, \theta, \phi_{1}, \phi_{2}, \phi_{3}\right)
$$


where $\phi_{i}$ and $\theta$ are the azimuth and zenith angles of the light sources. A first order Taylor expansion then yields an approximation of the error in gradient space $n=(-p,-q, 1)$ given the deviations $d I_{1}, d I_{2}, d I_{3}, d \theta, d \phi_{1}, d \phi_{2}, d \phi_{3}$ of the input data:

$$
d p=\sum_{i} \frac{\partial h_{1}}{\partial I_{1}} d I_{i}+\sum_{i} \frac{\partial h_{1}}{\partial \phi_{1}} d \phi_{i}+\frac{\partial h_{1}}{\partial \theta} d \theta, \quad d q=\sum_{i} \frac{\partial h_{2}}{\partial I_{1}} d I_{i}+\ldots
$$

For more than three light sources, a solution $h$ involves the pseudo inverse and computing the derivative is non-trivial. We therefore use a different strategy to analyze settings such as in the empirical experiment above. Assuming a ground truth normal $n_{\mathrm{GT}}$ and light directions $\hat{\Theta}=\left(\theta_{1}, \ldots, \theta_{6}\right), \hat{\Phi}=\left(\phi_{1}, \ldots, \phi_{6}\right)$, we model the observables with normal distributions

$$
I \sim \mathcal{N}\left(D(\hat{\Theta}, \hat{\Phi}) \cdot n_{\mathrm{GT}}, \sigma_{I}\right), \quad \Theta \sim \mathcal{N}\left(\hat{\Theta}, \sigma_{\theta}\right), \quad \Phi \sim \mathcal{N}\left(\hat{\Phi}, \sigma_{\phi}\right)
$$

where $D(\hat{\Theta}, \hat{\Phi})$ is the light matrix. Then, we draw random samples $\tilde{\Theta}, \tilde{\Phi}, \tilde{I}$ from each distribution and compute the resulting normal as

$$
n=n\left(\theta_{n}, \phi_{n}\right)=\frac{D(\tilde{\Theta}, \tilde{\Phi})^{\dagger} \tilde{I}}{\left\|D(\tilde{\Theta}, \tilde{\Phi})^{\dagger} \tilde{I}\right\|}
$$

where $D^{\dagger}$ denotes the pseudoinverse.

Repeating this sampling 10000 times lets us estimate the error for any given normal $n_{\mathrm{GT}}$ for a fixed light configuration $\hat{\Theta}, \hat{\Phi}$. We use the same set of normals and light directions as in the real experiment and set $\sigma_{I}=0.8 \%, \sigma_{\theta}=0.7^{\circ}, \sigma_{\phi}=1.5^{\circ}$ to reflect the findings in $\$ 2.3$. The results in Figure 2.6 show that deviations vary spatially over the sphere and are not isotropic. The accuracy decreases in the left and bottom regions because they are furthest away from the light directions. We also observe from Figure 2.6 that, given the uncertainties in the input data, we cannot expect to obtain much better results than a $1^{\circ}$ error. This puts the error of $2.5^{\circ}$ in the actual experiment into perspective and concludes our attempt to establish a baseline of quality.

\subsection{Integration of surface gradients}

Many photometric techniques recover the gradient of a surface, i.e. its orientation. On the other hand, it is often desirable to reconstruct the 

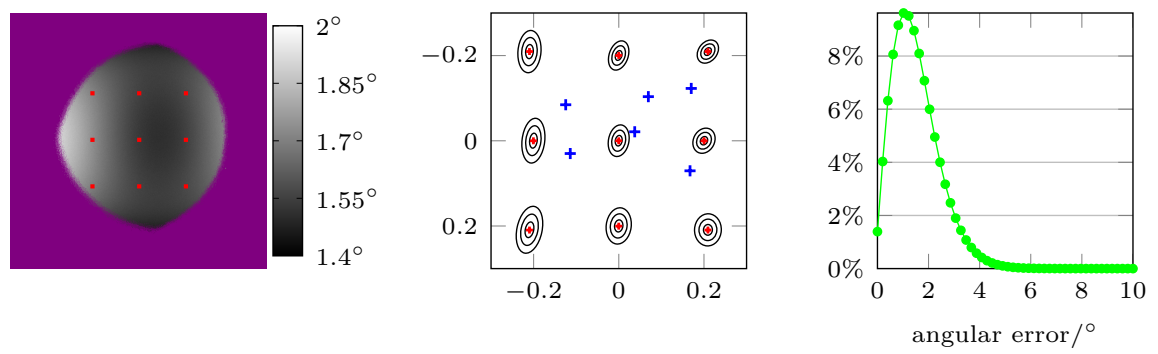

Figure 2.6: Results from simulated input errors through random sampling. Left: At each pixel, corresponding to the experiment in Figure 2.5, the angular error is averaged over 10000 random experiments. The nine pixels in red are selected for a closer examination. Center: For each of the exemplary normals marked in the left image, we fit a Gaussian to the stereographic projection of the reconstructed normals over 10000 random experiments. The ellipses correspond to the $1 \sigma, 2 \sigma, 3 \sigma$ intervals and show the spatially varying spread induced by the light configuration (blue) used in the experiment. Right: A histogram of all angular errors from the plot to the very left.

actual surface, and not just its derivative. Here, we summarize some key ideas for surface integration to complete the introductory chapter.

If the object is represented as a height field $(u, v, Z(u, v))$ over the image plane, its gradient field is defined as

$$
\nabla Z: \mathbb{R}^{2} \rightarrow \mathbb{R}^{2},(u, v) \mapsto\left(\begin{array}{c}
\frac{\partial Z(u, v)}{\partial u} \\
\frac{\partial Z(u, v)}{\partial v}
\end{array}\right)=\left(\begin{array}{c}
Z_{u}(u, v) \\
Z_{v}(u, v)
\end{array}\right) .
$$

What we obtain from photometric stereo is a vector field

$$
g: \mathbb{R}^{2} \rightarrow \mathbb{R}^{2},(u, v) \mapsto\left(\begin{array}{c}
p(u, v) \\
q(u, v)
\end{array}\right)
$$

or, interpreted as surface normals,

$$
N: \mathbb{R}^{2} \rightarrow \mathbb{R}^{3},(u, v) \mapsto \frac{1}{\sqrt{1+p^{2}+q^{2}}}(p(u, v), q(u, v),-1) .
$$

\subsubsection{Path integrals}

One strategy to recover the surface is to integrate the gradient field. Assume the photometric stereo reconstruction is perfect, i.e. $p=Z_{u}$, 
$q=Z_{v}$, and the gradient is differentiable. Then, the definition

$$
\tilde{Z}(u, v):=\int_{\gamma} g(w) \cdot \mathrm{d} w=\int_{a}^{b} g(\gamma(t)) \cdot \gamma^{\prime}(t) \mathrm{d} t
$$

is independent of the choice of $\gamma$ (which is an arbitrary path from $(0,0)$ to $(u, v))$. Conversely, it holds that $\nabla \tilde{Z}=g$. Thus, the path integral in Equation 2.18 is one way to obtain a solution. It is, however, not unique and might differ from the true solution by an additive constant because $\nabla(\tilde{Z}+c)=\nabla \tilde{Z}=g$. Examples of this approach can be found in the works by Wu and Li [1988 or Klette and Schluens 1996.

\subsubsection{Variational formulation}

In practice, the reconstruction is not perfect: the recovered gradients might differ from the true surface derivatives or they might not form an integrable vector field. It is therefore more common, $c f$. Durou et al. [2009, to define the solution in a variational framework and minimize

$$
E(\tilde{Z})=\iint\left(\tilde{Z}_{u}(u, v)-p(u, v)\right)^{2}+\left(\tilde{Z}_{v}(u, u)-q(u, v)\right)^{2} d(u, v)
$$

or similar error measures. Moreover, such a formulation can be incorporated directly into the reconstruction algorithm as shown by several shape from shading approaches, e.g. Horn and Brooks 1986. To avoid outliers and discontinuities, it can be beneficial to multiply the gradient terms with spatially varying weights as for example demonstrated by Agrawal et al. 2006. For practical computations it is often required to discretize the problem, e.g. in the spatial domain. Xie et al. 2014 show how this can be achieved by casting the integration as a mesh deformation task.

\subsubsection{Additional constraints}

Another approach to obtain absolute depth from gradients is to add additional information to guide the integration. This usually comes in the form of known depth values $\hat{Z}$ at sparse points or in the whole image area obtained through other means, e.g. laser scanning (Horovitz and Kiryati [2004]), active stereo methods (Nehab et al. 2005]), or depth 
cameras (Haque et al. 2014]). A possible extension of Equation 2.19 is, for example,

$$
E(\tilde{Z})=\iint\left(\tilde{Z}_{u}-p\right)^{2}+\left(\tilde{Z}_{v}-q\right)^{2}+(\hat{Z}-Z)^{2} d(u, v) .
$$

Combining normals and depth in such a way is a key part in several of the multi-view photometric stereo methods discussed in Chapter 8. 


\section{3}

\section{Foundations}

At lot of the techniques used today still rely in part on methods and concepts developed 30 years ago. It is instructive to briefly look at some of these early works and give credit to the ideas presented.

One of the first applications of photometry was in exploring the surface of the Moon. Rindfleisch [1965] derives and solves a differential equation for the distance of a surface point from the image plane that depends on the angles of incident and emitted light. His derivation is based on paths in the image plane, and the final integration depends on at least one depth value along a path to be known. The paths are chosen as straight lines that meet in the point given by the intersection of the image plane with the ray emanating from the camera center in the direction of the Sun. Rindfleisch assumes a certain reflection function for the surface of the Moon that essentially depends on two angles only. Furthermore, the camera geometry must be known and the discussion relies heavily on the Sun as known point light source.

Inspired by that work, Horn [1970] formulates the analytical shape from shading problem for arbitrary, but known, isotropic reflectance and known light sources. Again, this amounts to a first order partial differential equation. He transforms this into five ordinary differential 

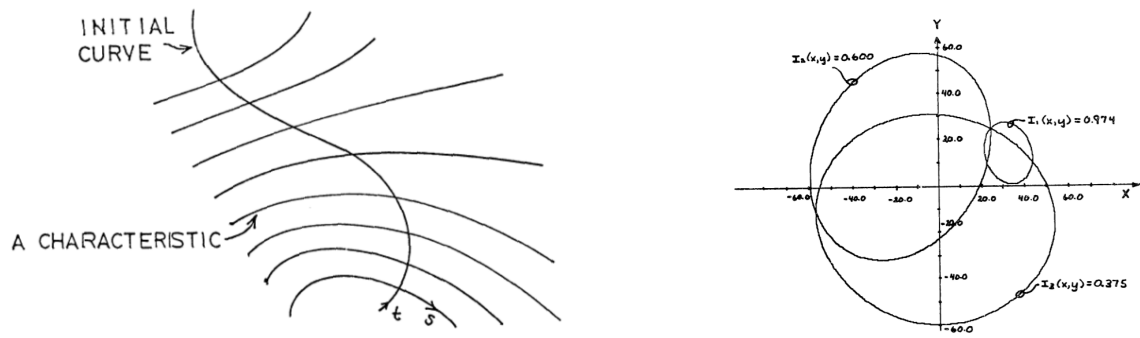

Figure 3.1: Left: Horn 1970 numerically integrates a system of differential equations along characteristic strips. They pass through an initial curve which is required as starting point. (C)1970 Massachusetts Institute of Technology. Reprinted, with permission, from Berthold K. P. Horn. Shape from shading: A method for obtaining the shape of a smooth opaque object from one view. Technical report, MIT Artificial Intelligence Laboratory, 1970). Right: Three iso-intensity curves in gradient space arising from different light configurations. A pixel that exhibits these three intensities corresponds to the gradient at the intersection point. (C)1977 Massachusetts Institute of Technology. Reprinted, with permission, from Robert J. Woodham. Reflectance Map Techniques for Analyzing Surface Defects in Metal Castings. PhD thesis, Massachusetts Institute of Technology, 1977).

equations that are solved along characteristic strips grown from an initial curve, see Figure 3.1. The theoretical formulation and the treatment of several special cases that simplify the occurring equations are truly pioneering work. Interestingly, Horn also mentions limitations of his imaging equipment. This aspect has become even more important today as computer vision is employed in consumer hardware with unknown characteristics.

In his PhD thesis - supervised by Horn-Woodham 1977] combines reflectance, illumination, and viewing geometry into a single function, a so called reflectance map, that relates surface orientation directly to image intensities

$$
I(u, v)=R\left(n_{u, v}\right) .
$$

In general, such a relationship can only be established if each object point receives the same incident illumination, has the same reflectance, and is observed from the same direction. This amounts to a distant light source, an untextured object, and an orthographic camera. The problem of inverting Equation 3.1 in a single image is similar to the work by Horn [1970], but instead of formulating a set of differential 
equations, Woodham defines additional constraints that can guide the inversion process. More important than the single image case is his extension of the photometric shape recovery problem to multiple images under varying illumination. He coins the term photometric stereo for a scenario of two or more independent equations

$$
\begin{gathered}
I_{1}(u, v)=R_{1}\left(n_{u, v}\right) \\
\vdots \\
I_{M}(u, v)=R_{M}\left(n_{u, v}\right)
\end{gathered}
$$

and explores this concept in the much cited paper Woodham 1980. Since a unit normal $n_{u, v}$ can be described by two angles, Equation 3.2 is an over-determined system of $M$ non-linear equations in two unknowns. Figure 3.1 shows how the intersection of the curves arising from each equation defines a solution in gradient space. In the case of a distant point light source $c$ shining from direction $\omega_{i}$ and Lambertian reflectance $\rho / \pi$, we obtain

$$
R_{i}\left(n_{u, v}\right)=\frac{\rho}{\pi} c\left\langle n_{u, v}, \omega_{i}\right\rangle .
$$

Inserting into Equation 3.2 yields the formulation in Equation 2.4 which is the basis of many subsequent works in this area.

Horn et al. 1978 pick up the concept of a reflectance map and enhance the previously mentioned methods to cope with spatially varying albedo in the Lambertion case by means of ratio images

$$
\left.\begin{array}{l}
I_{1}=\rho(u, v) R_{1}\left(n_{u, v}\right) \\
I_{2}=\rho(u, v) R_{2}\left(n_{u, v}\right)
\end{array}\right\} \rightarrow I_{12}:=\frac{I_{1}}{I_{2}}=\frac{R_{1}\left(n_{u, v}\right)}{R_{2}\left(n_{u, v}\right)}=: R_{12}\left(n_{u, v}\right) .
$$

The authors also give an overview about the possibilities of shape reconstruction from shading in one, two, or three images and compare these with stereo based methods. Several properties of the latter are complementary to those of photometric methods and provide the motivation for more recent, hybrid approaches.

One of the biggest hindrances for the broad application of photometric stereo is, however, its necessity for carefully controlled lighting conditions. Silver 1980 notes this problem in his master's thesis and 

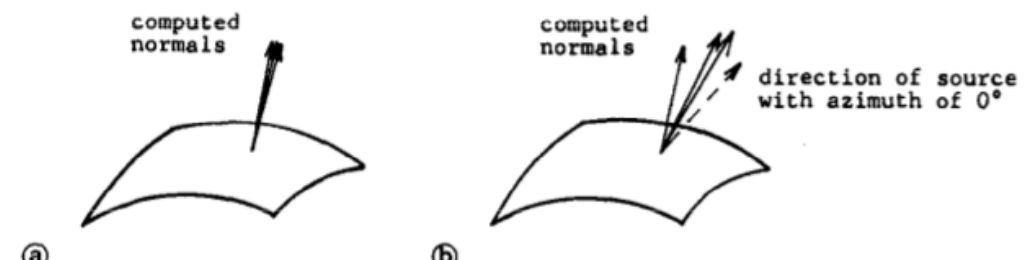

Figure 3.2: Four normals are computed from each combination of light triplets. Left: For a diffuse point all normals coincide. Right: If the point exhibits specularity under one of the light sources (dashed) the deviation among normals is higher. (Reprinted from E. North Coleman and Ramesh Jain. Obtaining 3-dimensional shape of textured and specular surfaces using four-source photometry. Computer Graphics and Image Processing, 18:309-328, 1982. Copyright 1982, with permission from Elsevier.).

develops an approach that works under varying but otherwise unknown light sources. It is based on a look-up scheme that relies on a reference object with the same reflectance as the target. This allows matching of intensity sequences between target and reference. He transfers the normals from the known reference based on the insight that two surface points with the same normal reflect the same amount of light. Several modern approaches draw inspiration from this orientation consistency as we will describe in $\$ 7.3$.

These techniques rely on a known reflectance map either through calibration or a reference object. Coleman and Jain [1982. present a technique that works for unknown, non-Lambertian objects without this restriction. They propose to use four known light sources even though three would suffice in the Lambertian case. This allows them to compute a solution for each of the $\left(\begin{array}{l}4 \\ 3\end{array}\right)=4$ combinations of three light sources. If the surface point is Lambertian, these are very similar and will all lead to the same albedo estimate. If the point exhibits a specularity under one of the sources, these estimates differ and the normal with the lowest albedo is accepted. Figure 3.2 illustrates this idea. The procedure relies on the assumption that a surface point behaves almost diffusely under most illuminations and that specularity only arises for a few constellations. Barsky and Petrou 2003 extend this approach using four known light sources with different color, which simplifies 
detection of highlights if the light source color is sufficiently different from the Lambertian surface color. If light source and surface color are similar, they propose an alternative method to identify a specular highlight or shadowed light source based on the direction of the erroneous initial surface normal estimate.

One of the early approaches to consider varying view point in addition to lighting conditions is presented by Hartt and Carlotto [1989. Their formulation is cast in a probabilistic framework and allows the inclusion of smoothness priors and a model of image noise. Its core is a comparison of observed intensities $I$ with renderings of hypothesized height fields $Z$. This is already quite similar to many modern approaches, as we will see in the following chapters. 


\section{Unknown lighting}

The first works on photometric methods, presented in the previous chapter, assumed that the light source is a known quantity. This is a realistic assumption in controlled settings such as an industrial factory or scientific laboratory. Lots of techniques have been developed to provide this kind of input data and we briefly listed some of them in $\$ 2.3$. Ultimately, we are interested in uncontrolled settings where this information is not accessible.

Performing an additional preprocessing step to estimate light source positions is cumbersome and introduces another source of errors. More importantly, equipment such as precise calibration spheres or positioning devices might not be available in a given acquisition setup. We will therefore look at uncalibrated photometric stereo techniques in \$4.1 and $\$ 4.2$ which recover the shape and lighting at one go.

These approaches are categorized according to the lighting model they employ. They all assume, however, an ideal camera model that might not be adequate for some real scenarios. \$4.3 presents generalizations that lessen the calibration effort and allow for a broader applicability. 


\subsection{Point light source}

One of the first methods to cope with the problem of unknown lighting was presented by Hayakawa 1994 and gave rise to other so called uncalibrated photometric stereo approaches. He arranges the radiance at $P$ pixels in $M$ images into a matrix

$$
L=\left(\begin{array}{ccc}
L_{1,1} & \ldots & L_{1, P} \\
& \ddots & \\
L_{M, 1} & \ldots & L_{M, P}
\end{array}\right)
$$

Assuming Lambertian reflectance, we know from Equation 2.4 that

$$
L=\underbrace{C \cdot D}_{=: T} \cdot \underbrace{N \cdot R}_{=: S}
$$

where the diagonal matrix $C \in \mathbb{R}^{M \times M}$ contains the source radiance, $D \in \mathbb{R}^{M \times 3}$ represents the light directions, $N \in \mathbb{R}^{3 \times P}$ is the stack of all normals, and $R \in \mathbb{R}^{P \times P}$ contains the reflection coefficients on its diagonal.

For given $L$, the goal is to find the matrix $S$. However, multiple candidates $\hat{T}, \hat{S}$ might fulfill

$$
L=\hat{T} \cdot \hat{S}
$$

In fact, any invertible $3 \times 3$ matrix $A$ defines a candidate pair $\hat{T}:=$ $T \cdot A, \hat{S}:=A^{-1} \cdot S$. Such a candidate pair can be obtained from $L$ using singular value decomposition. Additional constraints are necessary to find the actual $S, T$.

Hayakawa proposes to use six or more pixels with the same or known albedo, i.e.

$$
\rho_{1}^{2}=\left\|S_{1}\right\|^{2}=\left\langle S_{1}, S_{1}\right\rangle, \quad \ldots, \quad \rho_{6}^{2}=\left\|S_{6}\right\|^{2}=\left\langle S_{6}, S_{6}\right\rangle
$$

where $S_{i}$ are the corresponding columns of $S$. Choosing the same columns in a candidate matrix $\hat{S}$ yields

$$
\rho_{i}^{2}=\left\|S_{i}\right\|^{2}=\left\langle S_{i}, S_{i}\right\rangle=\left\langle A \hat{S}_{i}, A \hat{S}_{i}\right\rangle=\left\langle\hat{S}_{i}, A^{T} A \hat{S}_{i}\right\rangle .
$$

These equations constrain the entries of the symmetric matrix $B=$ $A^{T} A$, which has six degrees of freedom. Once this system of equations 
is solved, $A$ can be recovered - up to an unknown rotation - from $B$ using singular value decomposition. Multiplying the candidate with this transformation yields the final result $S=A \cdot \hat{S}$.

Techniques like the one just presented reconstruct a normal field $n(u, v)$ without considering an underlying surface. Instead, Belhumeur et al. 1999 formulate the problem in terms of a height field, i.e. a graph $(u, v, Z(u, v))$, with scaled normals

$$
n(u, v)=\left(\partial_{u} Z, \partial_{v} Z,-1\right) .
$$

To define a surface, $Z$ must satisfy the integrability constraint

$$
\partial_{u} \partial_{v} Z=\partial_{v} \partial_{u} Z
$$

which had already been used for shape from shading, e.g. by Horn and Brooks [1986] and Frankot and Chellappa [1988]. This provides constraints on any normal field that belongs to a surface. Not all transformed candidates $A \cdot \hat{S}$ are able to fulfill those. Belhumeur et al. 1999 show that for an integrable normal field $S$, the set of matrices $A$ that preserve this property is equivalent to

$$
\left(\begin{array}{ccc}
1 & 0 & -\mu / \lambda \\
0 & 1 & -\nu / \lambda \\
0 & 0 & -1 / \lambda
\end{array}\right)
$$

for parameters $\mu, \nu \in \mathbb{R}$ and $\lambda>0$. In practice, that means that an integrable normal field can be recovered by photometric methods only up to such a generalized bas relief transformation if no additional information is available. Figure 4.1 illustrates this finding with two differing constellations of light and surface that nevertheless yield the same image.

The bas relief transform not only applies to normals but also transforms the diffuse albedo. Alldrin et al. [2007] exploit the fact that many objects are composed of a small set of albedo values whose histogram gets broadened by a bas relief transform. They define an energy based on the entropy of the albedo distribution. Minimizing this energy yields the parameters of the correct transform.

Favaro and Papadhimitri 2012 look at the function $f(u, v):=$ $\left\langle n(u, v), \omega_{s}\right\rangle$ defined over the image domain. They discover that a maximum of this function constrains the parameters $\mu, \nu$ of the allowable 


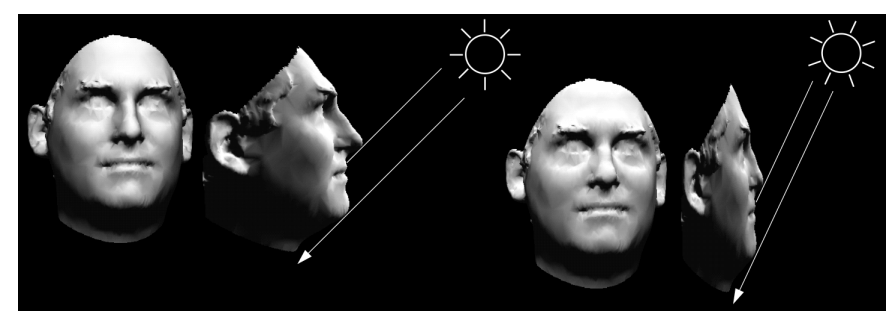

Figure 4.1: Without further constraints, multiple combinations of shape and lighting can result in the same image. For a differentiable surface, this ambiguity reduces to the so called generalized bas relief transform. (With kind permission from Springer Science+Business Media: Peter N. Belhumeur, David J. Kriegman, and Alan L. Yuille. The bas-relief ambiguity. International Journal of Computer Vision, 35:33-44, 1999. Figure 2.)

general bas relief transforms to a line and the parameter $\lambda$ to a semicircle over that line. Intersecting those curves in parameter space for multiple extrema yields a single point which completely describes the transform. Albedo variation can, however, make the detection of maxima difficult.

Finally, Drbohlav and Sára 2002] and Drbohlav and Chantler 2005] deviate from the strictly Lambertian reflectance assumption by adding a pure specular reflection term. This allows them to introduce the consistent viewpoint constraint, which states that for the actual $S, T$ all light directions $D$ must be reflected in the same viewing direction. Given the additional assumption of surface integrability, two specular pixels in two input images with different illumination conditions are sufficient in Drbohlav and Chantler 2005 to resolve the general bas relief ambiguity.

\subsection{General light source}

The works discussed above assume a distant point light source. That is an idealized setting. It is often violated, e.g. by ambient light that is hard to prevent in a real setup. Fortunately, the ideas introduced for point lights can easily be generalized to more complicated illumination.

Yuille and Snow 1997] use a similar matrix decomposition approach as Hayakawa but extend the shading model by a constant ambient term 


$$
L=\left(\begin{array}{ccc}
L_{1,1}+a_{1} & \ldots & L_{1, P}+a_{P} \\
& \ddots & \\
L_{M, 1}+a_{1} & \ldots & L_{M, P}+a_{P}
\end{array}\right)
$$

They enforce the integrability constraint to resolve the ambiguity in $A$ up to a generalized bas relief transform. The final solution is then defined by additionally assuming a light source with constant radiance.

Handling arbitrary unknown illumination is usually addressed by a decomposition of incoming radiance. If the incoming illumination $L_{s}$ can be decomposed into individual components

$$
L_{s}=\sum_{j=0}^{r} \lambda_{j} L_{s, j}
$$

this transfers to the outgoing radiance. Assuming Lambertian reflectance in Equation 2.2, we obtain

$$
\begin{aligned}
L & =\int_{\Omega} \frac{\rho}{\pi} \sum_{j} \lambda_{j} L_{s, j}\left(\omega_{\text {in }}\right)\left\langle n, \omega_{\text {in }}\right\rangle \mathrm{d} \omega_{\text {in }} \\
& =\sum_{j} \lambda_{j} \underbrace{\int_{\Omega} \frac{\rho}{\pi} L_{s, j}\left(\omega_{\text {in }}\right)\left\langle n, \omega_{\text {in }}\right\rangle \mathrm{d} \omega_{\text {in }}}_{=: L_{j}(n)}
\end{aligned}
$$

where $L_{j}$ is the radiance observed if the scene was illuminated just by $L_{s, j}$. Under these assumptions, the matrix in Equation 4.1 can be decomposed as

$$
L=\left(\begin{array}{ccc}
\lambda_{1,1} & \ldots & \lambda_{r, 1} \\
& \ddots & \\
\lambda_{1, M} & \ldots & \lambda_{r, M}
\end{array}\right) \cdot\left(\begin{array}{ccc}
L_{1}\left(n_{1}\right) & \ldots & L_{1}\left(n_{P}\right) \\
& \ddots & \\
L_{r}\left(n_{1}\right) & \ldots & L_{r}\left(n_{P}\right)
\end{array}\right)
$$

Basri and Jacobs 2001a argue that a decomposition into spherical harmonics represents a good approximation to the space of all possible images of a Lambertian object. A constant illumination yields the zero order harmonic image which corresponds to the surface albedo. The first order harmonic images are taken under cosine lighting for each of the three main axes and correspond to the respective components of 
the scaled normal at each pixel. Higher order decompositions reduce the approximation error.

In a second paper, Basri and Jacobs [2001b] show how to exploit these insights for photometric stereo reconstructions under arbitrary, unknown illumination. They use singular value decomposition to obtain a candidate factorization $L \approx \hat{\Lambda} \hat{L}_{s}$ as the best rank $r$ approximation of $L$. Similar to the discussion about the work by Hayakawa 1994, this factorization is only unique up to an $r \times r$ linear transformation. Basri and Jacobs 2001b] use a normalization constraint to reduce this ambiguity to a Lorentz transformation in the case $r=4$. Enforcing integrability again leads to a unique solution.

\subsection{Other generalizations}

The works discussed so far assumed an orthographic camera model like the majority of photometric stereo approaches. Papadhimitri and Favaro [2013] show how to incorporate a perspective camera model in a photometric reconstruction. More importantly, they find that a perspective formulation of the uncalibrated photometric stereo problem, when enforcing the integrability constraint, does not suffer from the bas relief ambiguity. Their results indicate also that an incorrect focal length or principal point can lead to strongly biased normals.

If in addition to a perspective camera the assumption of a directional light source does not hold, e.g. in endoscopic setups, the photometric stereo problem becomes even more general. Even if the position of the light source is known, its direction at each pixel is not because it depends on the depth of the observed point. Furthermore, light attenuation due to the $1 / r^{2}$ fall-off and angle dependent emittance of the light source lead to non-linearities in the equations. Collins and Bartoli 2012 solve this problem in a multi step optimization procedure that involves not only the normals as unknowns but also the depth at each pixel. A more rigorous formulation is derived by Mecca et al. 2014] who come up with a set of partial differential equations to encode the constraints on the depth mapping. They present two numerical schemes that recover the depth without separating it from its gradient, i.e. the 
normals. Integrating the differential equations requires the depth at one or more pixels to be known from which suitable integration paths are propagated outward.

None of these approaches considers the radiometric calibration of the camera. It is either assumed that calibration can be performed in a preprocessing step or is circumvented by using special cameras with linear response. Shi et al. [2010] propose an uncalibrated photometric stereo technique that also recovers the camera response curve. They exploit that the ratio between color channels for a single pixel is constant in a sequence of linear images but curved if a non-linear response is present. Measuring the non-linearity of the profiles in the RGB space allows them to define a minimization problem on the coefficients of a polynomial response model. For photometric stereo, they automatically select pixels with equal albedo and different normals which allows them to remove the bas relief ambiguity as in the approach by Hayakawa 1994.

Another aspect that is often overlooked are non-convex shapes. For example, a point in a deep crevice will not be visible from the entire hemisphere above its local tangent plane. In that case, some entries in the light matrix might be missing which we briefly discuss in Chapter 6 . Such effects are a lot harder to detect and handle in the presence of extended light sources. Then, the integration in Equation 4.11 would not be performed over all possible light directions and thus yield an attenuated radiance. If the light distribution can be split into a directional light source and a constant ambient term

$$
L_{s}=L_{s, \mathrm{dir}}+L_{s, \mathrm{con}}
$$

this attenuation is called ambient occlusion. Hauagge et al. 2013 estimate it from a set of images by studying a statistic over the pixel intensity distribution. They derive how this statistic relates to ambient occlusion and show its effectiveness in practice. Their approach could be a preprocessing step for photometric stereo techniques. 


\section{Unknown reflectance}

The appearance of a given object depends on its reflectance and the illumination. The approaches in Chapter 4 were focused on the case where the latter is unknown. They assume, however, that the surface reflects light according to the Lambertian model. We will now study the complementary setting in which the illumination is known or can be controlled and the reflectance is unknown.

Most real surfaces have reflectance properties that are neither purely Lambertian nor perfect mirrors. The linear equations in $\$ 2.2$ no longer hold and other reflection models have to be employed. This

makes a direct inversion of the underlying formulation in Equation 2.2 more challenging as we will see in $\$ 5.2$.

This chapter presents examples for the most common high-level strategies for shape reconstruction in the presence of unknown reflectance. Diffuse-specular separation in $\$ 5.1$ and parametric modeling in $\$ 5.2$ both have the disadvantage of model assumptions that might not be fulfilled by an arbitrary real-world BRDF. They have, however, been shown to work for a broad range of materials in practice. A benefit of parametric BRDF models is that they can be used to create novel impressions of the scene and that they fit into artists' editing pipelines. 
The approaches based on general invariants such as symmetries in $\$ 5.3$ only recover shape but are more general because they do not assume a fixed reflectance model. The downside is that they typically require special constellations of light source and camera during capturing.

\subsection{Diffuse and specular components}

A commonly followed path is to split the radiance into a diffuse and specular contribution

$$
L=\hat{\alpha}_{\text {diff }} \cdot L_{\text {diff }}+\hat{\alpha}_{\text {spec }} \cdot L_{\text {spec }} .
$$

Nayar et al. [1989] assume that the specular term consists of a single spike and approximate it with a delta function. That corresponds to a BRDF

$$
\rho\left(\omega_{\text {out }}, \omega_{\text {in }}\right)=\hat{\alpha}_{\text {diff }}+\hat{\alpha}_{\text {spec }} \cdot \frac{\delta\left(D_{R}-\omega_{\text {in }}\right)}{\left\langle n, \omega_{\text {in }}\right\rangle}
$$

in Equation 2.2 where $D_{R}=-\omega_{\text {out }}+2\left\langle\omega_{\text {out }}, n\right\rangle n$ is the reflection vector. They only study the two-dimensional case and reconstruct surface orientation $\varphi_{n}$ within the plane formed by $\omega_{\text {in }}$ and $\omega_{\text {out }}$. Using an extended light source $L_{s}\left(\varphi, \varphi_{s}\right)$ centered around direction $\varphi_{s}$ yields

$$
L=\alpha_{\text {diff }} \cdot \cos \left(\varphi_{s}-\varphi_{n}\right)+\alpha_{\text {spec }} \cdot L_{s}\left(2 \varphi_{n}, \varphi_{s}\right),
$$

where all angles are with respect to the viewing direction $D_{\text {out }}$.

Nayar samples this function $L\left(\varphi_{s, i}\right)$ for multiple known light source directions as shown in Figure 5.1. If the step size equals the extent of the light source, at most two samples $i, i+1$ are affected by the specular component because it is a delta peak. From these, $\alpha_{\text {spec }}$ and an estimate $\varphi_{n \text {,spec }}$ of the normal can be obtained. The remaining samples

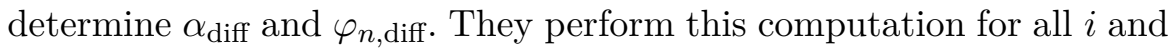
select the result where $\varphi_{n \text {,diff }}$ and $\varphi_{n \text {,spec }}$ are most similar.

Another way to deal with non-diffuse surfaces is to exploit polarization. As observed by Mersch [1984], specular reflections can often be removed using cross polarization. This requires placing polarization filters in front of both light sources and cameras and adjusting their polarization angle to be perpendicular (or crossed). In many cases, the 


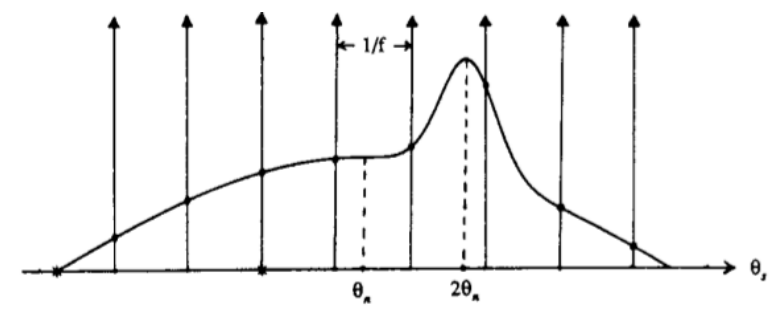

Figure 5.1: The image intensity at a surface point with normal $\varphi_{n}$ is a function of the source direction $\varphi_{s}$. If the sampling interval matches the extent of the light source, only the samples closest to the reflection vector $2 \varphi_{n}$ are affected by the specular component (C)1989 IEEE. Reprinted, with permission, from Shree K. Nayar, Katsushi Ikeuchi, and Takeo Kanade. Determining shape and reflectance of lambertian, specular, and hybrid surfaces using extended sources. In International Workshop on Industrial Applications of Machine Intelligence and Vision, 1989).

specular reflection itself partially polarizes light whereas light after diffuse reflection tends to be unpolarized Koshikawa 1979. Nayar et al. 1993. use this principle to estimate the specular term from multiple images obtained by rotating a polarization filter in front of the camera. Removing its contribution yields an image as if the object was diffuse.

Sato and Ikeuchi [1994b] extend the work of Nayar et al. [1989] to a simultaneous analysis of all three color channels in RGB space. Using a similar reflection model, they consider multiple samples in Equation 5.3 and arrive at the matrix expression

$$
\begin{aligned}
L & =\mathcal{D} \cdot \mathcal{K} \\
& :=\left(\begin{array}{cc}
\cos \left(\varphi_{s, 1}-\varphi_{n}\right) & L\left(2 \varphi_{n}, \varphi_{s, 1}\right) \\
\vdots & \vdots \\
\cos \left(\varphi_{s, M}-\varphi_{n}\right) & L\left(2 \varphi_{n}, \varphi_{s, M}\right)
\end{array}\right) \cdot\left(\begin{array}{ccc}
\alpha_{\mathrm{diff}, R} & \alpha_{\mathrm{diff}, G} & \alpha_{\mathrm{diff}, B} \\
\alpha_{\mathrm{spec}, R} & \alpha_{\mathrm{spec}, G} & \alpha_{\mathrm{spec}, B}
\end{array}\right) .
\end{aligned}
$$

This model corresponds to the dichromatic reflection model introduced by Shafer [1984 who notes that the specular vector $\left(\alpha_{\mathrm{spec}, R}, \alpha_{\mathrm{spec}, G}, \alpha_{\mathrm{spec}, B}\right)$ usually has the same color as the light source. Sato and Ikeuchi estimate this vector from several pixels of different color. Then, the diffuse component $\left(\alpha_{\mathrm{diff}, R}, \alpha_{\mathrm{diff}, G}, \alpha_{\mathrm{diff}, B}\right)$ is obtained from similar arguments as exploited by Nayar et al. [1989] - most samples capture only the diffuse contribution. Finally, they recover the matrix $\mathcal{D}$ from Equation 5.4 by inverting the color matrix $\mathcal{K}$. To get the 
actual orientation $\varphi_{n}$, however, knowledge of the light source directions $\varphi_{s, i}$ is required.

Mallick et al. 2005 also assume a dichromatic reflection model but propose to rotate the original RGB color space into an SUV color space so that the $\mathrm{S}$ channel of the color space corresponds to the color $\left(c_{R}, c_{G}, c_{B}\right)^{T}=C$ of the light source:

$$
L_{\mathrm{SUV}}=A \cdot L
$$

with rotation $A \in S O(3)$ and $A \cdot C=[1,0,0]^{T}$. The two remaining channels $\mathrm{U}$ and $\mathrm{V}$ depend only on the diffuse reflectance component and can be analyzed using standard Lambertian photometric stereo methods. This allows the method to be applied to arbitrary colored or textured surfaces as long as the color of the light source $C$ is known or can be estimated. Purely white surfaces can, however, not be recovered since in this case the $\mathrm{U}$ and $\mathrm{V}$ channels contain no signal.

\subsection{More complex BRDF models}

Instead of approximating specular reflectance with a delta peak, more sophisticated BRDF models allow for broader lobes and smeared out highlights. Tagare and deFigueiredo [1991] give theoretical insights on photometric stereo under such $m$-lobed reflectance maps

$$
\begin{aligned}
& R_{i}\left(n_{u, v}\right)= \sum_{j=1}^{m-1} \rho_{j} \Phi_{j}\left(p_{i, j}^{T} n_{u, v}\right)+\rho_{m} \\
& \Phi_{j}: \mathbb{R}^{+} \rightarrow \mathbb{R}^{+} \quad \text { positive and monotonically increasing, } \\
& p_{i, j} \in \mathcal{S} \quad \text { directions in the plane spanned by } \omega_{\mathrm{in}, i}, \omega_{\mathrm{out}}, \\
& p_{i, j}^{T} n_{u, v} \quad \text { scalar product with the normal, }
\end{aligned}
$$

that encompass fore- and backscattering cases. An example configuration is shown in Figure 5.2. The authors confirm experimentally that a simple Lambertian assumption for the reconstruction of nonLambertian objects leads to high errors and thus motivate the use of more sophisticated models.

While Tagare and deFigueiredo explicitly invert the photometric stereo equation similar to $\$ 2.2$, another approach is to employ non- 


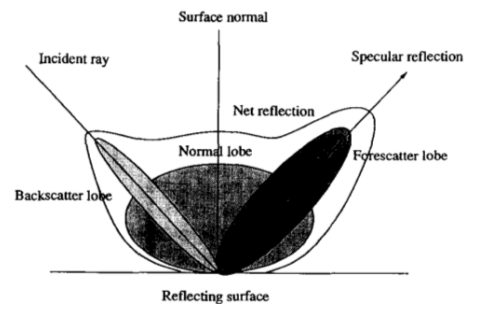

Figure 5.2: Rough surfaces exhibit a mix of different reflection phenomena. They can be modeled as a weighted sum of individual contributions such as diffuse and specular reflection or backscattering lobes. (C)1991 IEEE. Reprinted, with permission, from Hemant D. Tagare and Rui J. P. deFigueiredo. A theory of photometric stereo for a class of diffuse non-lambertian surfaces. IEEE Transactions on Pattern Analysis and Machine Intelligence, 13, 1991).

linear optimization techniques to minimize the image intensity error

$$
\underset{N, \rho}{\arg \min }\|I-\mathcal{M}(N, \rho)\|,
$$

where $\mathcal{M}$ can be any model of light transport in the scene. However, such an optimization is not straightforward due to the possibly large amount of parameters and their non-linear relationship.

Goldman et al. 2005 alternatingly estimate the reflectance properties $\rho$ of a parametric BRDF model by Ward [1992 and the surface orientation in the form of a normal field $N$. Varying the reflectance parameters $\rho_{u, v}$ at each surface point would lead to lots of unknowns, require a huge amount of images, and affect the robustness negatively. Instead, they recover only a small set of basis BRDFs $\rho_{j}$ and per-pixel mixing coefficients $\gamma_{u, v, j}$ similar to Lensch et al. 2003:

$$
\rho_{u, v}=\sum_{j} \gamma_{u, v, j} \rho_{j}
$$

The optimization still requires a good initialization, which they obtain using Lambertian photometric stereo.

Aittala et al. 2013 also estimate spatially varying reflectance using a parametric BRDF model - based on sums of Gaussians - and nonlinear optimization. In contrast to Goldman et al. 2005], they derive one set of parameters at each pixel. Their technique relies on an extended light source, e.g. a LCD display, that projects a series of illu- 
mination patterns onto the target. It is primarily a BRDF acquisition setup but also recovers surface normals for almost planar objects.

An even more challenging case of unknown reflectance is considered by Dong et al. 2014a. They study materials, such as skin or soap, that exhibit subsurface scattering. Then, reflectance at a single point can no longer be described by a four dimensional BRDF and applying photometric stereo leads to blurred results. Instead, they model outgoing radiance as a convolution with a bidirectional subsurface scattering distribution function (BSSRDF). A non-linear optimization that involves a blind deconvolution step recovers normals that are much closer to the ground truth.

The complexity of the employed BRDF models in these approaches leads to non-linear formulations that are often hard to optimize. Shi et al. 2012b address this by introducing a simplified reflectance model based on a biquadratic factorization of isotropic BRDFs. They show that it approximates the low-frequency components of other parametric models and measured BRDFs. If the light source positions are known, surface normals and BRDF parameters can be reconstructed by simply alternating two linear least squares optimizations.

Ikehata and Aizawa 2014 also transform the problem of recovering normals and BRDF into a representation that is better suited for optimization. They base their derivations on the multi-lobed reflectance model introduced by Chandraker et al. 2011.

$$
\rho_{u, v}=\sum_{j=1}^{m} \Phi_{j}\left(p_{j}^{T} n_{u, v}\right)
$$

where $\Phi_{j}$ are nonlinear functions and $p_{j}$ are directions along which these functions are concentrated. The authors prove that if $p_{j}$ lies in the plane spanned by the viewing and lighting direction, the whole image formation can be represented by a bivariate function $f$

$$
L_{i}(u, v)=h_{i}\left(\left\langle n_{u, v}, \omega_{\text {in }, i}\right\rangle,\left\langle\omega_{\text {in }, i}, \omega_{\text {out }, i}\right\rangle\right)
$$

with certain monotonicity constraints. These ensure the existence of an unknown function $g$ such that

$$
\left\langle n_{u, v}, \omega_{\text {in }, i}\right\rangle=g\left(\left\langle\omega_{\text {in }, i}, \omega_{\text {out }, i}\right\rangle, L_{i}(u, v)\right)
$$


and thus separate the unknown normal from the nonlinear function. Approximating $g$ using Bernstein basis polynomials then leads to a constrained linear problem.

Note that the above BRDF models only approximate the real reflectance behavior and may break down in specific cases (typical examples are anisotropic reflectance or retro-reflectance). One thus needs to always check whether these models and techniques are applicable to the specific reconstruction scenario at hand.

\subsection{Invariants}

A different approach to handle complex BRDFs is to exploit general invariants, such as symmetries and physical properties, in the image formation model or capture setup. Such techniques are usually independent of any explicit parametric reflectance model. This is an advantage in terms of generality but can be a disadvantage if editing of scene appearance is desired.

Zickler et al. 2002] use Helmholtz reciprocity, which states that a $\mathrm{BRDF}$ is invariant if the incoming and outgoing directions are swapped: $\rho\left(\omega_{\text {in }}, \omega_{\text {out }}\right)=\rho\left(\omega_{\text {out }}, \omega_{\text {in }}\right)$. Their setup consists of a camera and a light source which can be exchanged to acquire a reciprocal pair. The intensity - ignoring irradiance fall-off due to the light source distanceof a surface point in both images of a pair yields

$$
\left.\begin{array}{c}
I_{r}=\rho\left(\omega_{\text {in }}, \omega_{\text {out }}\right) \cdot\left\langle n, \omega_{\text {in }}\right\rangle \\
I_{l}=\rho\left(\omega_{\text {out }}, \omega_{\text {in }}\right) \cdot\left\langle n, \omega_{\text {out }}\right\rangle
\end{array}\right\} \Longrightarrow 0=\langle n, \underbrace{I_{r} \omega_{\text {out }}-I_{l} \omega_{\text {in }}}_{=: w}\rangle .
$$

For multiple pairs, the vectors $w$ can be stacked into a matrix $W$ that fulfills $0=n^{T} W$ and $\operatorname{rank} W=2$. Note that $\omega_{\text {in }}, \omega_{\text {out }}$ are assumed to be in a coordinate system local to the surface point $X$ and depend on its position in relation to the camera/light. Using the rank of $W(X)$ as an indicator of surface depth, this method recovers the 3D position $X$. Once the correct depth is found, the normal is given by the kernel of $W(X)$.

Capturing reciprocal pairs requires a careful calibration and camera/light placement. Zickler [2006] shows how such a calibration can 


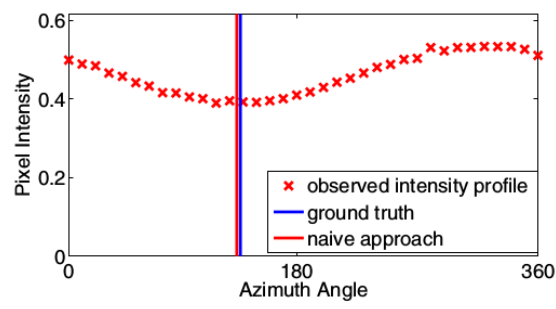

Figure 5.3: Intensities samples for a surface point with isotropic BRDF under a light source that moves around the optical axis. The samples exhibit a symmetry around a particular light position, parametrized by the azimuth angle $\varphi_{g}$. The normal of the point lies in the plane define by $\varphi_{g}$ and the viewing direction. (C) 2013 IEEE. Reprinted, with permission, from Zhenglong Zhou, Zhe $\mathrm{Wu}$, and Ping Tan. Multi-view photometric stereo with spatially varying isotropic materials. In IEEE Conference on Computer Vision and Pattern Recognition [2013]).

be obtained from features in the reciprocal images themselves. It remains, however, necessary to swap the light and camera for each pair. Delaunoy et al. [2010] use the same concept as Zickler et al. [2002] but formulate an energy over a global 3D model instead of separate depth maps.

In many cases, other kinds of symmetries can be exploited besides Helmholtz reciprocity. One example is bilateral symmetry for isotropic BRDFs, i.e., symmetry across the plane of incidence

$$
\begin{aligned}
\rho\left(\theta_{\text {in }}, \phi_{\text {in }}, \theta_{\text {out }}, \phi_{\text {out }}\right) & =\rho\left(\theta_{\text {in }}, \phi_{\text {in }}, \theta_{\text {out }}, \phi_{\text {in }}+\left(\phi_{\text {out }}-\phi_{\text {in }}\right)\right) \\
& =\rho\left(\theta_{\text {in }}, \phi_{\text {in }}, \theta_{\text {out }}, \phi_{\text {in }}-\left(\phi_{\text {out }}-\phi_{\text {in }}\right)\right),
\end{aligned}
$$

which was introduced in Marschner's Ph.D. thesis (Marschner 1998]). Alldrin and Kriegman 2007 use the concept of bilateral symmetry based on so called isotropic light pairs. This requires a circle of light positions - parametrized by the angle $\varphi$ - centered about the optical axis of the camera. For a single pixel, the radiance distribution $L(\varphi)$ given by these light positions exhibits a symmetry around a certain angle $\varphi_{g}$ depicted in Figure 5.3 . This angle and the viewing direction define a plane that contains the normal. The authors show that this information is sufficient to recover iso-depth contours of the surface. Further constraints are necessary to obtain the unique normal or the absolute depth. 

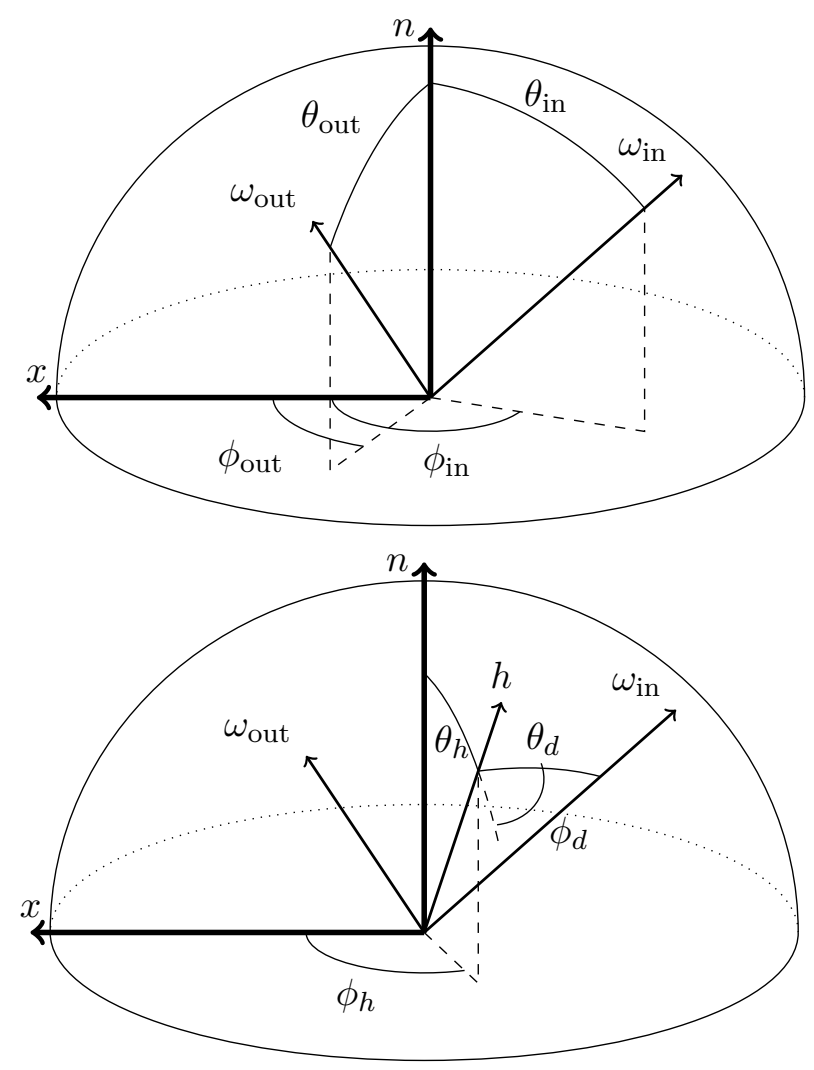

Figure 5.4: Two common parametrizations of a BRDF. Top: Incoming $\omega_{\text {in }}$ and outgoing $\omega_{\text {out }}$ directions are encoded as azimuth in the tangential plane and zenith angle with respect to the normal. Bottom: The incoming direction is encoded by difference angles $\left(\theta_{d}, \phi_{d}\right)$ with respect to the halfway vector $h=\left(\omega_{\text {out }}-\omega_{\text {in }}\right) /\left\|\omega_{\text {out }}-\omega_{\text {in }}\right\|$ which is itself represented as zenith and azimuth angles $\left(\theta_{h}, \phi_{h}\right)$. $\omega_{\text {out }}$ is implicitly defined by reflecting $\omega_{\text {in }}$ around $h$.

Using this technique as initialization, Alldrin et al. 2008 present a system that recovers not only surface orientation but also BRDFs. This makes it possible to render novel views of captured objects under different illumination. They make the additional assumption that the reflectance is well represented by a bi-variate function. Based on the halfangle representation introduced by Rusinkiewicz [1998], cf. Figure 5.4. Romeiro and Zickler 2010b show that this holds if the BRDF does not change too much for rotations of the incoming and outgoing 
directions around the halfway vector

$$
\rho\left(\theta_{h}, \phi_{h}, \theta_{d}, \phi_{d}\right) \approx \rho\left(\theta_{h}, \phi_{h}, \theta_{d}, \tilde{\phi}_{d}\right) .
$$

The technique by Alldrin et al. then relies on a set of basis materials which are represented as data samples and thus do not depend on a parametric model. The normals and material weights at each pixel and the basis BRDFs for the full object are found through an alternating optimization.

As mentioned above, bilateral symmetry alone determines only the azimuth angle of the normals. Shi et al. 2012a give an example of an additional constraint to also recover the zenith angle. It is based on the assumption of a BRDF as in Equation 5.12 with a dominant lobe concentrated around the halfway vector $\Phi\left(h^{T} n\right)=\tilde{\Phi}\left(\theta_{h}\right)$. In general, the monotonicity $\theta_{h_{1}}>\theta_{h_{2}} \Rightarrow \tilde{\Phi}\left(\theta_{h_{1}}\right)<\tilde{\Phi}\left(\theta_{h_{2}}\right)$ is broken for an incorrect normal estimate. The approach requires light directions to be known and distributed equally on the hemisphere to detect such a violation. Holroyd et al. 2008, also investigate symmetries similar to Alldrin and Kriegman [2007], but additionally consider tangents. This makes it possible to study anisotropic materials. They define a measure of symmetry for a pair $(n, t)$ of normal and tangent under three possible reflection operations. Minimizing that measure at each pixel yields a local coordinate frame which can, for example, be used to fit a parametric BRDF model. Their technique, however, requires thousands of images with known light position.

The methods discussed so far required the light source positions to be known explicitly. Chandraker et al. 2011] again use light sources that move on a circle around the optical axis but do not require their actual position. They exploit image derivatives both in the spatial and in the temporal domain, i.e. neighboring pixels and successive light directions, to define a ratio that is constant over time and independent of the BRDF. From this invariant, it is possible to recover the direction of the gradient and, similar to the idea of Alldrin and Kriegman 2007, the iso-depth contours. The advantage of this technique is that it works for unknown light source positions as long as they fulfil the circular motion assumption. A disadvantage in practice is that the reconstruction involves higher-order derivatives of image intensities, which tend to be 


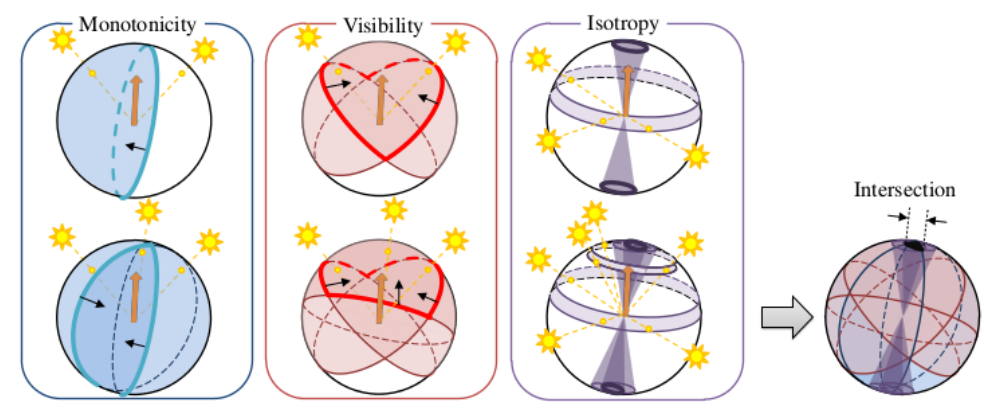

Figure 5.5: Narrowing down the possible normals by combining the solution spaces of many weak constraints works even for non-linear camera responses. (C)2010 IEEE. Tomoaki Higo, Yasuyuki Matsushita, and Katsushi Ikeuchi. Consensus pho- tometric stereo. In IEEE Conference on Computer Vision and Pattern Recognition [2010]).

unstable due to noise. Furthermore, additional input data is needed to completely determine depth and normals.

As in Chapter 4, all techniques discussed here, except the one by Alldrin and Kriegman [2007, rely on pixel values that are linearly related to scene radiance. Higo et al. 2010] exploit three properties present in many BRDFs which even hold for observations $o$ under an unknown camera response function. From known light source directions $\omega_{i}$, they define a monotonicity constraint

$$
o_{i}>o_{j} \Longleftrightarrow\left\langle\omega_{i}-\omega_{j}, n\right\rangle>0,
$$

a visibility constraint

$$
\left\langle\omega_{i}, n\right\rangle>0
$$

and an isotropy constraint

$$
o_{1} \simeq \ldots \simeq o_{k} \quad \Longrightarrow \quad \operatorname{Var}_{i}\left(\left\langle n, \omega_{i}\right\rangle\right) \text { minimal }
$$

Figure 5.5 visualizes this abstract formulation. While each of the constraints is relatively weak, e.g. visibility is valid for all normals in a half-space, combining them in a consensus manner over lots of light directions yields very good results. 


\section{6}

\section{Outlier handling}

No matter whether an approach is able to cope with unknown illumination as in Chapter 4 or with unknown reflectance as in Chapter 5 , it always assumes that the input data adheres to a fixed model or behaves in a certain way. If these assumptions are repeatedly violated-beyond statistical measurement errors - in a real scenario, the results become unusable.

Several effects, e.g. shadows, interreflections, image noise, nonlinear camera response, non-ideal light sources, etc., are not accounted for in the simple model employed by many photometric stereo methods. They are often treated as outliers, but sometimes they can even be exploited explicitly to obtain additional information. We will discuss some examples of the latter in $\$ 6.1$ and then look at more general outlier treatment in $\$ 6.2$.

The ideas from $\$ 6.2$ can also be exploited in the context of Chapter 5 if non-Lambertian reflectance is considered as an outlier. This is similar in spirit to the concept of Coleman and Jain [1982] introduced in Chapter 3. It requires, however, that sufficiently many images show a surface point without specularity. 


\subsection{Explicit treatment}

\subsubsection{Interreflections}

Nearly all approaches neglect global illumination effects, i.e. light that reaches a surface patch not directly from the source but after one or more bounces at other surfaces. The actually observed radiance then has a direct and indirect component $L=L_{\text {direct }}+L_{\text {indirect }}$ from which only $L_{\text {direct }}$ follows the model in Equation 2.3 . Nayar et al. [1990] discuss the impact of interreflections on photometric reconstruction techniques. They assume the scene to consist of infinitesimal, planar patches of Lambertian reflectance similar to radiosity approaches in computer graphics (Goral et al. 1984). The geometric relations between patches can be encoded in a kernel matrix $B \in \mathbb{R}^{P \times P}$ :

$$
B=\left(\begin{array}{ccc}
0 & b_{1,2} & \cdots \\
b_{2,1} & 0 & \\
\vdots & & \ddots
\end{array}\right)
$$

where $b_{i, j}$ encodes the transfer of energy between the interreflecting surface patches $i$ and $j$. So the observed radiance can be written as:

$$
L=(\mathrm{I}-R B)^{-1} L_{\text {direct }}=(\mathrm{I}-R B)^{-1} R N D
$$

where I is the identity matrix, the diagonal matrix $R \in \mathbb{R}^{P \times P}$ contains the albedo and $N \in \mathbb{R}^{P \times 3}$ the normal of each patch, and $D \in \mathbb{R}^{3 \times M}$ the light source directions. As hinted at by Figure 6.1, a standard photometric stereo technique would result in false estimates $\tilde{N}, \tilde{R}$ :

$$
\tilde{R} \tilde{N}:=L \cdot D^{-1}=(\mathrm{I}-R B)^{-1} R N \quad \Leftrightarrow \quad R N=(\mathrm{I}-R B) \cdot \tilde{R} \tilde{N} .
$$

Nayar et al. solve this problem by iteratively updating the left hand side with calculations of the right hand side based on estimates from the previous iteration. Chandraker et al. [2005] study a similar problem in the context of uncalibrated photometric stereo. They are able to show that interreflections actually resolve the generalized bas relief ambiguity. 


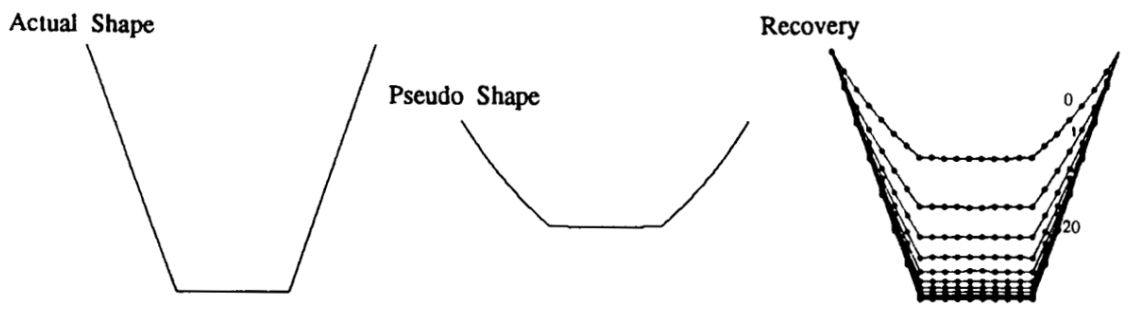

Figure 6.1: Impact of interreflections on shape reconstruction. Left: A surface point on the concave shape does not only receive light from the actual source but also light that was reflected from other parts of the object. Middle: Traditional photometric stereo underestimates the slope of the walls and reconstructs the wrong shape. Right: Iterative corrections of the photometric stereo result by incorporating the form factors of planar patches on the object (c)1990 IEEE. Reprinted, with permission, from Shree K. Nayar, Katsushi Ikeuchi, and Takeo Kanade. Shape from interreflections. In IEEE International Conference on Computer Vision, 1990).

\subsubsection{Shadows}

Shadows are another source of inconsistencies. One distinguishes cast shadows - generated because light towards the surface point $X$ is blocked by another point $\tilde{X}$ - and attached shadows - occurring because the surface faces away from the light source, i.e. $\left\langle n, \omega_{\text {in }}\right\rangle \leq 0$. Daum and Dudek 1998 reconstruct surface height based on cast shadows from known point light sources. They derive several constraints based on geometric reasoning such as if point $X$ is shadowed by $\tilde{X}$, then all surface points on the connecting line must lie below the ray from $X$ to $\tilde{X}$.

Chandraker et al. 2007. use similar constraints for 3D surface reconstruction in their ShadowCuts paper. The approach requires at least four input images of a Lambertian scene illuminated from a combination of four or more light sources, so that each surface point is observed under at least three different, non-shadowed illuminations. Starting from the observation that light source visibility is piecewise constant over a surface, they propose an efficient graph cut-based approach, which determines the per-pixel light source visibility labeling that minimizes the photometric stereo error of the scene. Their variational surface reconstruction approach combines photometric stereo with the constraints given by the shadow labeling. 
Kriegman and Belhumeur [2001] instead study attached shadows. They reconstruct the light positions and normals at intersection points of shadow boundaries. Okabe et al. 2009 exploit the binary pattern (shadowed/non-shadowed) at a pixel created by a large sequence of light positions. The similarity between two such patterns is related to the angular difference of the corresponding normals. This insight allows them to recover surface orientation using dimensionality reduction inspired by Sato et al. 2007] (see $\$ 7.3$ for a detailed description of this paper).

In a real scene, cast and attached shadows will be present at the same time. Sunkavalli et al. 2010] observe that the light source visibility is constant in subregions of the surface. The intensities in each region lie in a three-dimensional subspace as exploited by uncalibrated photometric stereo methods. The overall intensity matrix thus consists of several rank three submatrices which can be obtained using subspace clustering techniques.

\subsection{General deviations}

\subsubsection{Exploiting high inlier to outlier ratios}

In contrast to approaches that explicitly treat specific effects, another line of work tries to detect any deviation from the local, Lambertian shading model and then applies photometric stereo only to the remaining intensities. This usually requires many images to ensure that a surface point is observed sufficiently often without shadows or specular behavior.

Such a dense photometric stereo technique is proposed by $\mathrm{Wu}$ and Tang 2006. They formulate a probabilistic image formation model that includes a binary inlier/outlier map as hidden variable. For a single pixel in $M$ images, they compute a set of $\left(\begin{array}{c}M \\ M-1\end{array}\right)$ normals and represent their distribution as a covariance matrix $B$. The algorithm estimates an optimal $B$ by weighting down observations that are likely to be outliers according to the map. After updating the weights, the whole process is repeated until convergence. Another example of this category is given by Verbiest and Gool 2008 who also employ expectation maximization 


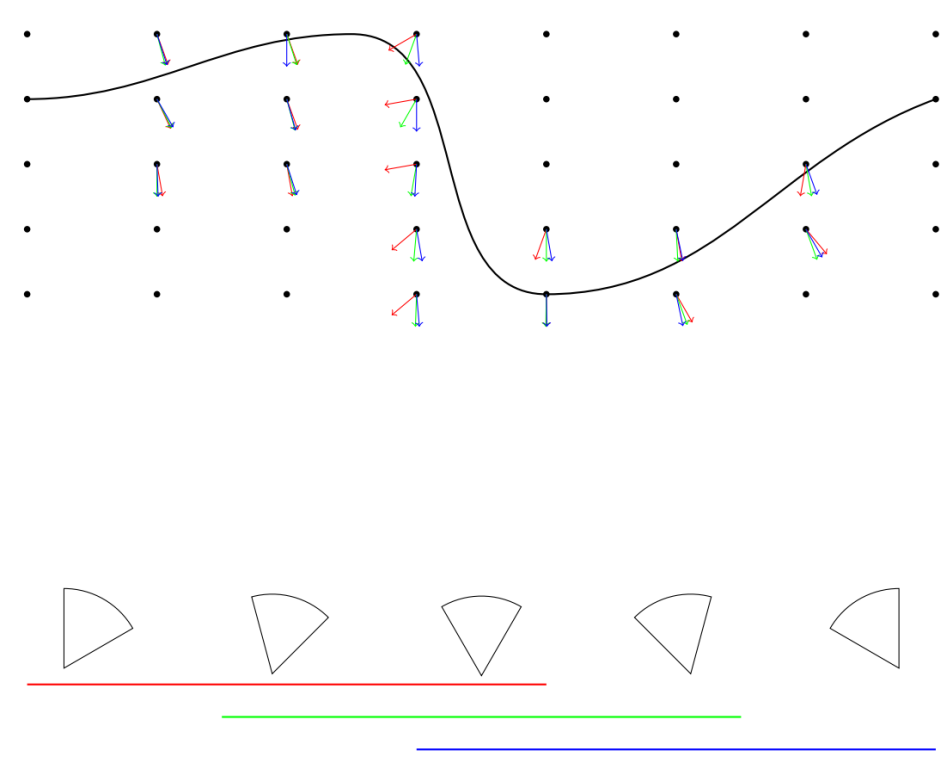

Figure 6.2: A multi-view setting with 5 camera and light positions. Three of the ten possible combinations are highlighted. For selected voxels, the normals computed from these triplets are visualized. (Reprinted, with permission, from Mate Beljan. Multi-view photometric stereo using a normal consistency approach. Master?s thesis, Technische Universität Darmstadt, 2011.)

but treat the normal map as a hidden variable. Instead of the perpixel approach of $\mathrm{Wu}$ and Tang, they describe the likelihood of outlier intensities with per-image histograms.

Beljan et al. 2012 also consider sets of normals for a single point computed from $\left(\begin{array}{c}M \\ 3\end{array}\right)$ subsets of all available images, but do so in a multiview setting. The simplified example in Figure 6.2 shows the spread of these normals at different point. For each image triplet, Beljan et al. 2012 compute the set of inlier images whose observations are consistent with the hypothesized normal. Instead of studying the distribution of these normal hypotheses, they apply RANSAC to find the one that has the largest support. The number of inliers then provides a cue to decide whether a voxel belongs to the true surface or not. This leads to good results for the normals but turns out to be not very discriminative in terms of absolute geometry. 


\subsubsection{Matrix corruptions}

A slightly different perspective on photometric stereo with outliers is provided by techniques that analyze the observation matrix for errors and missing entries. Wu et al. [2010 interpret the problem as a low rank matrix recovery task in the presence of sparse corruptions. More formally, they augment the matrix decomposition in Equation 4.2 with an error matrix $E$ that contains all outliers due to shadows or specularities:

$$
L=T \cdot S+E=: K+E .
$$

As we have seen before, the rank of $T \cdot S$ is at most three for a Lambertian surface. Under the assumption that outliers occur infrequently, $E$ is sparse and the task becomes

$$
\underset{K, E}{\arg \min }\left[\operatorname{rank}(K)+\alpha\|E\|_{0}\right], \quad \text { s.t. } L=K+E
$$

where $\|\cdot\|_{0}$ is the number of non-zero entries - and thus not a true norm. Wu et al. 2010 replace this formulation with a convex optimization problem

$$
\underset{K, E}{\arg \min }\left[\|K\|_{\star}+\alpha\|E\|_{1}\right], \quad \text { s.t. } L=K+E,
$$

which can be minimized iteratively using Lagrange multipliers with additional penalty terms (Augmented Lagrange Multiplier method, $c f$. Bertsekas and Rheinboldt 2014]). If the locations of shadows are known beforehand, they enforce the constraints only on the remaining entries.

Once $K$ is recovered, standard tools such as singular value decomposition can be applied to obtain the factorization $S, T$. Other approaches in this field of research, e.g. by Okatani and Deguchi [2007], Okatani et al. 2011], Eriksson and van den Hengel [2010], directly optimize for the decomposition

$$
\underset{S, T}{\arg \min }\|H \odot(L-T \cdot S)\|
$$

with a known matrix $H$ encoding missing entries and $\odot$ being the component-wise multiplication. Such a technique can also be applied to cope with outliers in calibrated photometric stereo as, for example, shown by Ikehata et al. 2012. 


\section{7}

\section{Unknown lighting and reflectance}

Shape reconstruction of Lambertian surfaces under unknown illumination is possible as discussed in Chapter 4 . Techniques that handle more complex BRDFs are presented in Chapter 5 but require known light sources or special capture setups. Reconstructing objects with complex BRDFs under unknown illumination is a much harder problem because both constituents interact in a complex way to form the observed appearance.

To a certain extent, ideas from the previous chapters can be applied to this setting with some modifications. For example, \$7.1 extends the idea of diffuse and specular separation in $\$ 5.1$ by applying one of the techniques for general light sources presented in $\$ 4.2$ to the diffuse component. Similarly, $\$ 7.2$ is related to the handling of parametrized BRDF models as discussed in $\$ 5.2$. Incorporating the unknown lighting in such a formulation adds, however, several degrees of freedom that make a robust solution non-trivial. A more general approach is to directly study similarities in the sequence of intensity values at single pixels. Works that exploit such so called appearance profiles are discussed in $\$ 7.3$. 


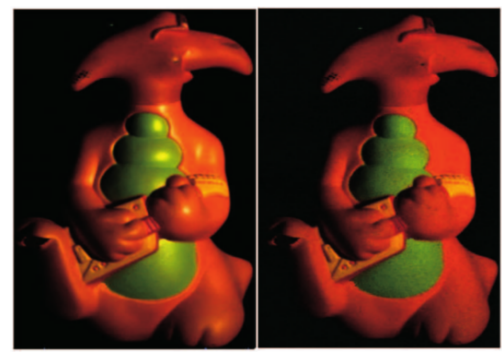

Figure 7.1: Techniques to remove the specular highlights from an image (left) can be employed as preprocessing. Only the diffuse component (right) is considered for photometric stereo (C)2005 IEEE. Reprinted, with permission, from Robby T. Tan and Katsushi Ikeuchi. Separating reflection components of textured surfaces using a single image. IEEE Transactions on Pattern Analysis and Machine Intelligence, $27: 178-193,2005)$.

\subsection{Diffuse specular separation}

Low-rank factorizations of the intensity matrix as in uncalibrated photometric stereo are not easily possible in the case of non-Lambertian reflectance. A straightforward way to deal with this problem is to separate the diffuse and specular contributions using a technique such as the chromaticity based method by Tan and Ikeuchi [2005 shown in Figure 7.1. Applying one of the methods in Chapter 4 to the diffuse component then yields the desired normal field.

Tan et al. 2007 propose such a system and show that the generalized bas relief ambiguity can be resolved from the additional information present in the specular component. The approach is based on isotropy and reciprocity constraints derived from two or more images, but Tan and Zickler [2009], Tan et al. [2011] show how this can even be reduced to one. Wu and Tan 2013 use a similar strategy of diffusespecular separation and find the optimal bas relief transform through a constraint on the structure of the BRDF. This requires objects with sufficient distribution of normal directions, e.g. a sphere, and an isotropic BRDF that exhibits a symmetry around the halfway vector. 


\subsection{Parametric models}

A different approach is to define a mathematical model of image formation that encompasses all unknowns and to directly minimize a suitable error, e.g. image intensity differences

$$
\underset{N, \rho, L_{s}}{\arg \min }\left\|I-\mathcal{M}\left(N, \rho, L_{s}\right)\right\| .
$$

This is similar in spirit to $\S 5.2$ but adds the incoming light distribution $L_{s}$ to the unknowns. Such an approach comes at the same costs as discussed there: complex optimization problem, susceptibility to local optima, and dependence on a parametric model that might not represent all real-world scenarios.

An example is the technique by Georghiades 2003. It uses a simplified Torrance-Sparrow BRDF (Torrance and Sparrow [1967]) and a single distant light source. The optimization is, however, restricted to a single BRDF and only allows the diffuse albedo to vary spatially. $\mathrm{He}$ is able to show that the generalized bas relief ambiguity is resolved for specular surfaces in almost all cases if at least four images are captured.

\subsection{Appearance profiles}

We have seen another way to approach photometric reconstruction problems in general in Chapter 5: symmetries and general properties of BRDFs. In the setting discussed here, reasoning about symmetries suffers from unconstrained lighting. A much more common strategy is to exploit general relations between observation vectors or appearance profiles that encode the temporal appearance variation of a single pixel, i.e. columns in Equation 4.1. One example is the work of Shi et al. [2010], that uses appearance profiles to introduce a self-calibrating photometric stereo technique (see the detailed description of the paper in $\$ 4.3$.

A recurring concept in this context is orientation consistency which states that points with similar normals have similar appearance. Assuming an orthographic camera, i.e. constant $\omega_{\text {out }}=v$ for all surface 
points, the radiance of a point $X$ with normal $n$ is given by

$$
f^{-1}(I)=L=\int \rho\left(v, \omega_{\text {in }}\right) L_{s}\left(\omega_{\text {in }}\right)\left\langle n, \omega_{\text {in }}\right\rangle \mathrm{d} \omega_{\text {in }}
$$

according to Equation 2.2. The right hand side depends only on the normal and BRDF but not on the 3 D position. Thus, for a point $\tilde{X}$ on the surface with the same normal and BRDF as $X$ the radiance is the same. Moreover, if the radiance is the same, the pixel intensities are equal, too- even for a non-linear response curve $f$.

Hertzmann and Seitz 2003 place one or more example objects of known geometry, usually a sphere, in the scene to obtain reference profiles

$$
W_{i}=\left(\begin{array}{ccc}
I_{1, i, 1} & \ldots & I_{1, i, k} \\
\vdots & & \vdots \\
I_{M, i, 1} & \ldots & I_{M, i, k}
\end{array}\right)
$$

of $k$ basis BRDFs. For a pixel $j$ on the target object, they match its appearance profile

$$
w_{j}:=\left(\begin{array}{lll}
I_{1, j} & \ldots & I_{M, j}
\end{array}\right)^{T}
$$

against linear combinations $W_{i} m$ of the reference profiles to find a corresponding point

$$
\underset{i}{\arg \min }\left\|W_{i} m_{j}-w_{j}\right\|^{2}, \quad m_{j}:=W_{i}^{\dagger} w_{j}
$$

on the reference. Based on orientation consistency, both points are then likely to have the same normal. Since normals for the reference object are known, they can simply be transferred to the target, see Figure 7.2 . This approach has the added advantage to require only a minimal amount of calibration. Ackermann et al. [2010] remove the need for an explicit example object by replacing it with a partial reconstruction of the scene using multi-view stereo techniques. This bootstrapping allows them to apply the example-based photometric stereo idea even in scenarios where physical access to the scene is difficult such as for Internet images.

If the light source positions vary smoothly, derivatives of the appearance profiles can be computed. Koppal and Narasimhan 2006 obtain 

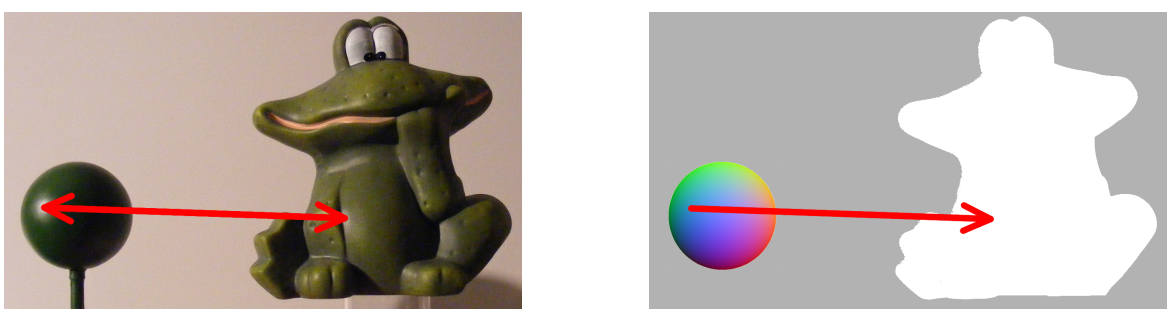

Figure 7.2: The concept of appearance matching for shape reconstruction. Left: Finding pixels with similar intensity (usually in a series of images) on the target and a reference sphere. Right: Because of orientation consistency, normals can be transferred between matches.

the extrema of appearance profiles from such derivatives under an otherwise unknown light source. They show that most of these extrema are due to constant constellations of viewing direction, light source, and surface normal. This insight makes the set of extrema locations well-suited features for the clustering of surface orientations independent of the BRDF. This process alone, however, cannot recover the relationship between iso-normal clusters, e.g. one facing left and one facing right. Other techniques are needed to assign absolute orientation to the clusters. Those can, however, benefit from the initial clustering as prior knowledge.

Sato et al. [2007] look at appearance profiles as a whole and not just at the locations of extrema. They argue that similar profiles - seen as vectors in $M$-dimensional space - correspond to similar normals, which they justify based on the studies of the Torrance-Sparrow BRDF model (Torrance and Sparrow [1967]). Since normals are defined on the unit sphere $\mathcal{S}$ in $\mathbb{R}^{3}$, this implies that the set of profiles $\mathcal{M}$ is actually a twodimensional manifold in $\mathbb{R}^{M}$. Thus, there should exist an embedding $\Phi: \mathbb{R}^{M} \rightarrow \mathbb{R}^{3}$ with $\Phi(\mathcal{M}) \subset \mathcal{S}$ that preserves the intrinsic structure of $\mathcal{M}$, i.e. points that are close in terms of $\mathcal{M}$ correspond to normals that are close on $\mathcal{S}$. The notion of closeness on $\mathcal{M}$ is expressed by the geodesic distance, which can be approximated by Euclidean distances along a sequence of neighboring profiles.

Sato et al. [2007] then employ the Isomap non-linear dimensionality reduction technique by Tenenbaum et al. 2000 to obtain the embed- 
ding $\Phi$ and afterward force the result vectors to have unit length. The normal map thus recovered contains only relative orientations, e.g. a global rotation or reflection are not considered, but can be made unique if additional constraints, such as contours, are applied. The advantages of this technique are its generality and a certain robustness against non-linear camera responses. It makes, however, the assumption of a spatially constant ratio between diffuse and specular contributions and requires hundreds of images.

Lu et al. [2013] extend the work by Sato et al. [2007] in several ways. They show empirically - using the MERL (Matusik et al. 2003]) database of measured BRDFs - that the geodesic distance of the profiles is proportional to the angular difference of normals

$$
d_{\text {geod }}\left(w_{i}, w_{j}\right) \propto \operatorname{acos}\left(\left\langle n_{i}, n_{j}\right\rangle\right), \quad \text { for }\left|\left\langle n_{i}, n_{j}\right\rangle\right|>1-\delta,
$$

and that the proportionality factor depends only on the material. They also propose a way to estimate this factor from intensity samples obtained under uniformly distributed, but unknown, point light sources. In combination this allows to recover the $P \times P$ matrix $N^{T} N$ containing the dot products of all pairs of normals. Instead of dimensionality reduction techniques, their recovery step is based on factorizing this matrix and removing the arising ambiguity by means of the integrability constraint. 


\section{Multi-view settings}

Most photometric techniques assume a fixed view point. Information about the orientation and reflectance of a surface is recovered from changes in the illumination. This, however, restricts the result to a single perspective and does not allow a reconstruction of a full, e.g. $360^{\circ}$, object model. Furthermore, varying view points provide additional information. For example, observing an object from the front and side lets us estimate its absolute position in the scene and not just the surface orientation. Multiple views also introduce redundancy in surface coverage, which can be exploited to increase robustness.

If the illumination is fixed while the camera moves, we leave the scope defined in $\$ 1.1$. Nevertheless, \$8.1 discusses some stereo algorithms because they can seen as complementary tools to photometric reconstructions as noted by Horn et al. [1978]. Stereo often performs poorly on surfaces that exhibit only little texture variation. On the other hand, it provides a good estimate of the absolute geometry and not only recovers surface orientation that has to be integrated as in $\$ 2.5$. Photometric techniques work well even in uniform regions but can suffer from low-frequency distortions. Some of the approaches in later sections will therefore combine both techniques to counter their respective disadvantages. 
We will categorize the techniques relying mainly on shading variations according to the magnitude of the assumed motion of the scene components. For small, differential changes in camera and lighting discussed in $\$ 8.2$, the situation is not too different from a fixed camera and can be addressed by per-pixel perturbations as in optical flow methods. A second strategy is to recover an initial proxy and adjust its shape based on photometric reasoning. This idea also gets applied in $\$ 8.3$ where camera baselines are much wider and the changes in lighting are not restricted to smooth variations.

\subsection{Fixed illumination}

Reconstructions from two or more views are commonly addressed by stereo or multi-view stereo methods. The idea is to find properties that are invariant - or almost invariant - to view-point changes such as the color of a Lambertian surface point $X$ under constant illumination. If $X$ projects to pixel $\tau_{1}(X)$ in the first image and $\tau_{2}(X)$ in the second, their color will be the same. For a hypothesized point $\tilde{X}$ that does not lie on the surface, the color of its projections $\tau_{1}(\tilde{X})$ and $\tau_{2}(\tilde{X})$ will differ in general. Thus, it is possible to detect the correct position by checking the consistency of projections with respect to the invariant. Figure 8.1 illustrates this concept. For increased robustness, small surfaces patches are often used in favor of single points. The image intensities for a projected patch $\mathcal{P}$ in two cameras $i, j$ are then compared using metrics such as the sum of squared differences

$$
d_{i, j}(\mathcal{P})=\sum_{X \in \mathcal{P}}\left[I_{i}\left(\tau_{i}(X)\right)-I_{j}\left(\tau_{j}(X)\right)\right]^{2}
$$

or the normalized cross correlation. For multiple image pairs, a hypothesis is either retained or discarded based on an aggregation of the scores $d_{i, j}$.

Seitz et al. 2006 give a good introduction to the aspects of different multi-view stereo approaches and provide an overview over the literature in this field. We note that almost all techniques assume a Lambertian surface. Exceptions are the works by Jin et al. [2003], Soatto et al. 2003, Jin et al. 2005, which use a rank constraint on the radiance 


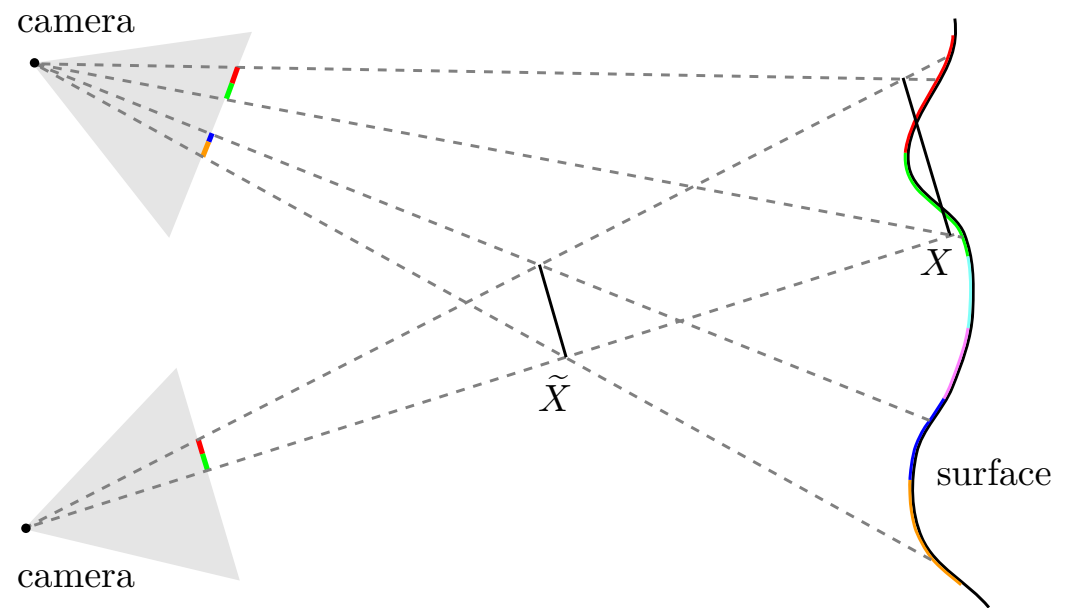

Figure 8.1: Concept of patch-based stereo. A hypothetical patch at the wrong position $\tilde{X}$ leads to different color values in both cameras. At the correct position $X$, the projection yields a red and a green pixel in both cameras.

tensor to handle specular reflectance, or the work by Yu et al. [2004], who compare input images with renderings of an object under a simplified Torrance-Sparrow model (Torrance and Sparrow 1967]). These approaches require the illumination to be constant and do not exploit the additional information conveyed by variations in shading.

\subsection{Differential motion}

Some of the first steps towards multi-view reconstructions that consider shading are connected to the analysis of densely sampled video sequences. Carceroni and Kutulakos 2001 use multiple video streams at calibrated positions to recover the motion, shape, and Phong reflectance parameters (Phong [1975]) of deformable surfaces under known point light sources.

Zhang et al. 2003 introduce a framework that encompasses the similarity of patches seen from multiple cameras and their appearance change caused by varying illumination. The authors recover the direction of a distant light source, the surface normals, and absolute depthi.e. the distance from a camera center - in an alternating optimization. 
A general theory for shape reconstruction from differential object motion is derived by Chandraker et al. 2013. It extends optical flow to objects of unknown BRDF under arbitrary illumination.

These works rely on relatively dense temporal sampling and smooth object motion in order to linearize the relationship of image intensities $\Delta I=I_{i}-I_{i+1}$. Thus, it is questionable whether they are also suited for wide-baseline, multi-view setups.

Lim et al. 2005, while also considering video frames as input, do not explicitly exploit this linearization. They initialize a piece-wise planar surface by triangulating tracked scene points. Projecting it into all views yields an intensity matrix, which can be factorized to obtained normals from uncalibrated photometric stereo. The positions of the tracked points allow a disambiguation of the general bas relief transform. Then, the surface is updated by integrating the normals into a depth map. This surface is again projected into all views to create a new intensity matrix. The authors iterate these steps until convergence.

Joshi and Kriegman 2007] follow the same line of thought. They derive a consistency measure for Lambertian surfaces which allows them to estimate a coarse depth map. Projecting this depth map into all images then yields an intensity matrix, which can be factorized. They resolve the ambiguities by comparing the pseudo normals with the normals obtained by differentiating the depth map. Lastly, the normals and depth map are both integrated into a final surface.

Their consistency measure is based on hypothesizing planar patches, which they project into all images and form an intensity matrix for each patch. As we have seen in $\$ 2.2$, this matrix has rank three for a Lambertian surface. If the patch was at an incorrect depth, the error of a rank three approximation to this matrix should be high. Minimizing this error combined with a straightforward smoothing does not yield high quality depth maps, but is sufficient as initialization.

We observe that these techniques alternate between a depth estimation and a normal estimation step. This is because photometric stereo requires known pixel correspondences to associate the intensities to a surface point. In traditional photometric techniques, these are trivially defined by the fixed view point assumption. 


\subsection{Wide baseline}

The technique by Joshi and Kriegman 2007 was used in a video-based reconstruction setup but does not explicitly exploit differential motion. This example leads us to another group of algorithms that do not make such strong assumptions about the changes in object, camera, or light positions. We summarize the setups of some these in Table 8.1. They allow for wider baselines between views and increased freedom in light source movement.

Another difference is that the approaches discussed before employ a local surface representation, i.e. defined per view. We will now look at methods that rely on a global geometric model of the surface. This has the advantage of providing readily available occlusion information while the surface is deformed by the optimization.

\section{Voxel carving}

Weber et al. 2002 use a voxel representation and consider objects on a turntable. They carve away voxels for which the predictions of the Lambertian model - given light source positions - disagree with the recorded intensities even for the best fitting normal. The estimated albedo and normals can then be used to render the scene with novel lighting.

Yang et al. 2003 develop a consistency measure based on the behavior in color space of a surface point observed from multiple views: it varies linearly from the diffuse color to the color of the light source. In contrast to other voxel-based formulations, the approach additionally employs a smoothness constraint. This is not straightforward to define for a voxel representation, and the authors resort to extending the disparity gradient introduce by Burt and Julesz [1980], which is originally defined between two images only. Their method does not recover normals and actually considers mostly a setting with fixed illumination.

Treuille et al. 2004 also reconstruct a voxel representation. They consider multiple light directions and require one or more example objects in the scene similar to Hertzmann and Seitz 2005. Their consistency measure compares appearance profiles for a candidate voxel 


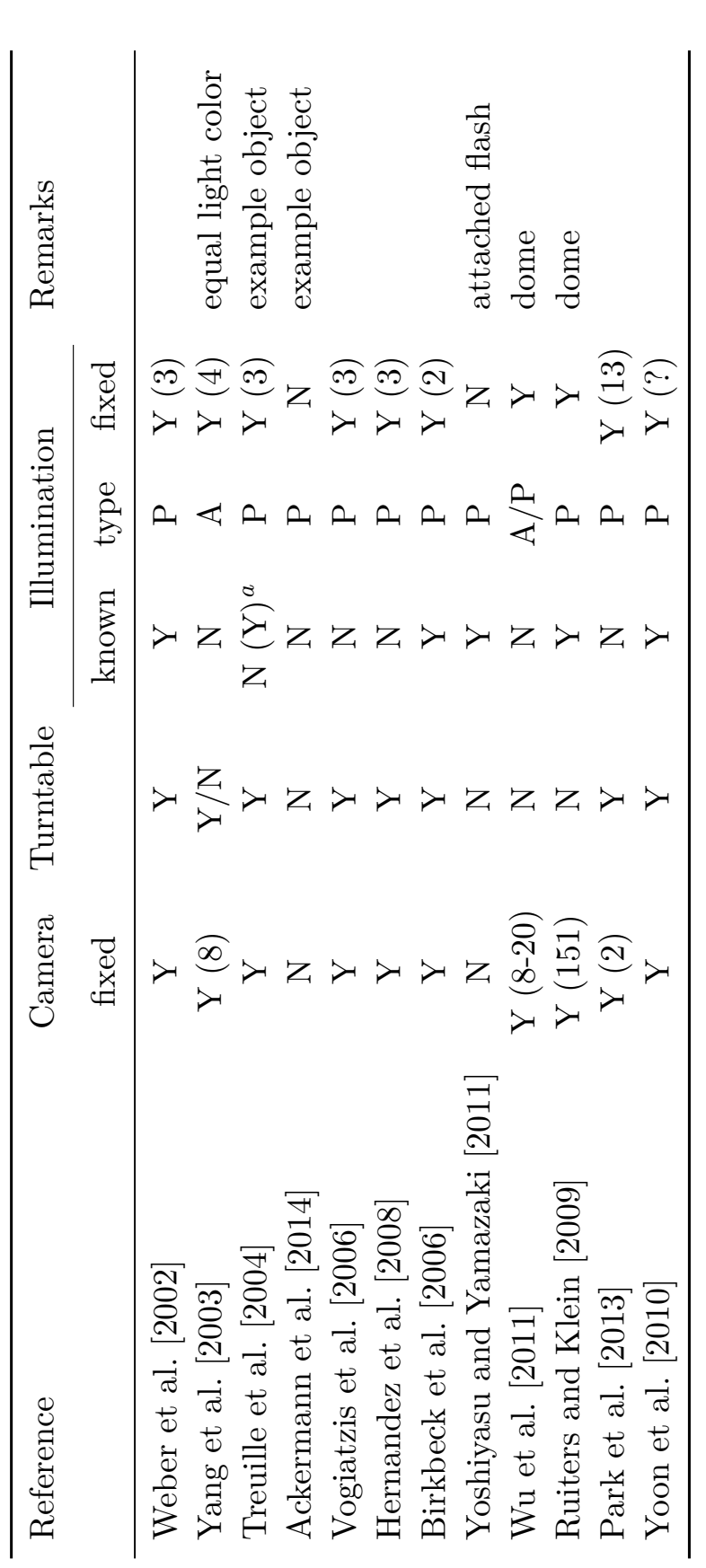

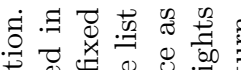

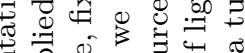
家 $\frac{0}{b 0}$ 至

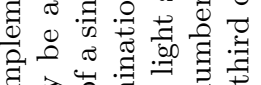

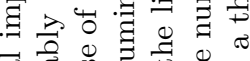
ซึ

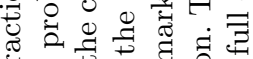

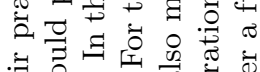

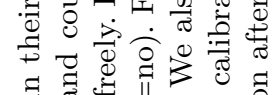

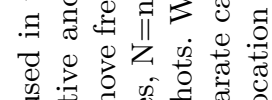
कै:

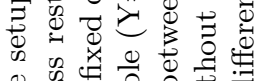

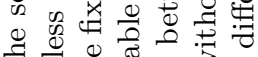
$\exists \cong \pi^{\circ}$ च

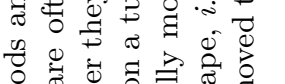
कै

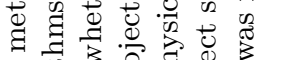

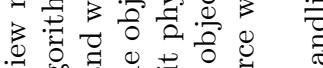

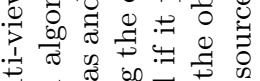

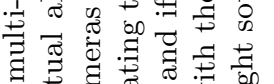
0 䒕

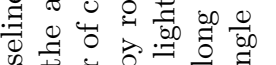

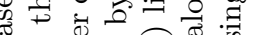

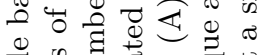

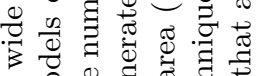
a :

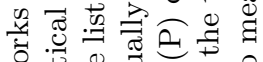

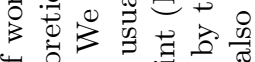

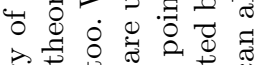

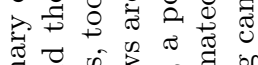

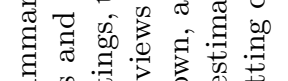

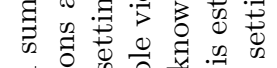

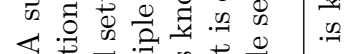

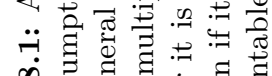
$\infty$

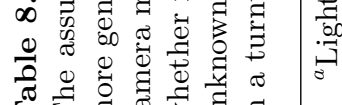



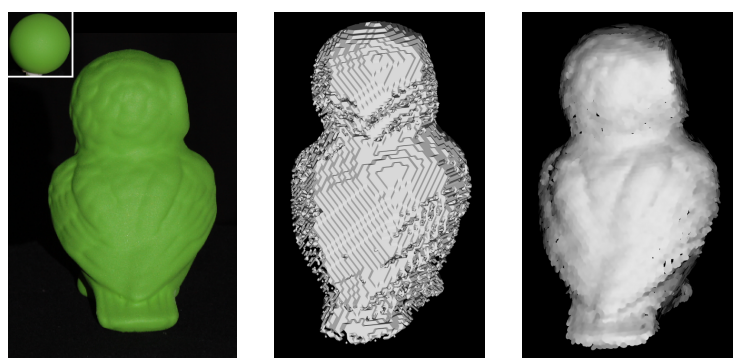

Figure 8.2: Illustrating the voxel-based reconstruction by Treuille et al. 2004. Left: One of the input images taken from multiple view points and with varying illumination. The inset shows the reference sphere that is photographed along with the target object under the same lighting conditions. Middle: Due to the discrete voxel grid, the actual surface geometry cannot represent fine details. Right: The detail information encoded by the normals can be exploited for rendering.

against those on the reference objects. Figure 8.2 illustrates that the reconstructed normals contain much more details than the coarse voxel approximation. This information can, however, only be exploited during rendering because normals and voxels are not fused into a single surface representation. A further restriction concerns the camera positions, which need to be separated from the scene by a hyperplane to ensure the correct order during voxel processing. These restriction are addressed by Ackermann et al. [2014] who use a continuous, local representation instead of a global voxel model. There approach might, however, lead to slight inconsistencies between views.

\section{Mesh deformation}

Vogiatzis et al. [2006] and Hernandez et al. [2008] represent the global model as a triangle mesh with attached normals, which circumvents the drawback of the method by Treuille et al. [2004]. The approach in both works assumes Lambertian surfaces and treats specular reflections as outliers. It relies heavily on object silhouettes to recover the camera parameters, light directions $D_{k}$, and an initialization of the mesh $\mathcal{P}$. The mesh is then projected into the images to obtain the intensities $I_{i, k}$ per face and light direction. From these, normals $N$ are computed 
with a standard photometric stereo approach

$$
\underset{N}{\arg \min } E_{\text {photo }}(N ; \mathcal{P})=\underset{N}{\arg \min } \sum_{i} \sum_{k}\left(\left\langle n_{i}, D_{k}\right\rangle-I_{i, k}\right)^{2} .
$$

This step is alternated with a vertex displacement that forces the face normals $\tilde{N}$ defined by the mesh vertices $\mathcal{P}$ to conform with the reconstructed normals $N$

$$
\underset{\mathcal{P}}{\arg \min } E_{\text {disp }}(\mathcal{P} ; N)=\underset{\mathcal{P}}{\arg \min } \sum_{i}\left\|\tilde{n}_{i}(\mathcal{P})-n_{i}\right\|^{2} .
$$

Birkbeck et al. 2006 also filter out specular reflections during shape recovery but fit a specular Blinn-Phong model (Blinn 1977]) once the final geometry is available. Thus, the final output of this algorithm is not just the object shape but also reflectance parameters. To achieve this, they obtain the input images and the required light calibration from a very controlled capture system. All three techniques can draw on initializations that are already quite close to the true surface.

Yoshiyasu and Yamazaki 2011] show that a similar approach can succeed with a much simpler initialization, e.g. a set of spheres approximating the object as in Figure 8.3 . It also operates on mesh vertices, but converts them into an implicit surface and back to a mesh during the optimization. This allows to handle a greater variability in topologyimplicit surfaces can, for example, self-intersect. Similar to Hernandez et al. [2008, their technique requires silhouettes to be extracted. In fact, if the mesh deformation is constrained solely by silhouettes - ignoring the photometric term completely - it already provides very good results. The photometric cue is much weaker and only serves to add some details. Such combinations are also used in dynamic performance capture systems as proposed by Ahmed et al. 2008], Vlasic et al. [2009].

While these approaches rely on silhouettes, the general idea of deforming an initial reconstruction based on photometric information can also be applied in other settings. Wu et al. 2011] reconstruct an initial mesh using multi-view stereo and refine it with normals from uncalibrated photometric stereo. Another way of initialization is to assume a mostly planar target as suggested by Ruiters and Klein [2009. They represent geometry variations as a height field and recover spatially 

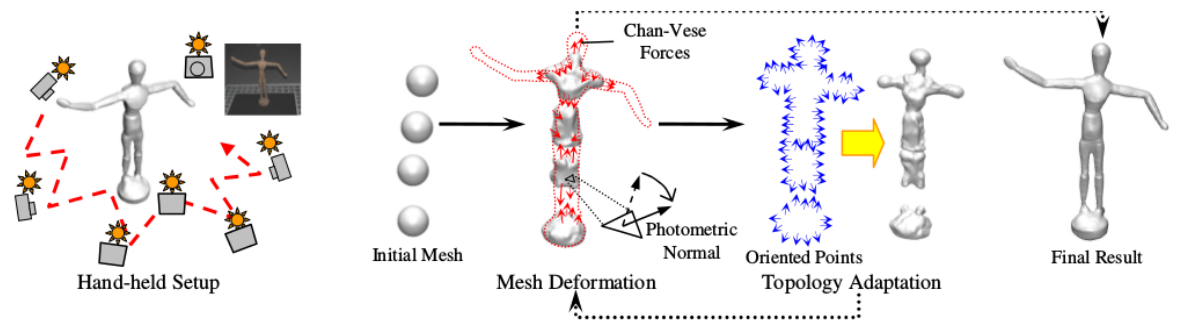

Figure 8.3: Overview of the multi-view reconstruction approach by Yoshiyasu and Yamazaki 2011. A basic mesh gets deformed iteratively to fulfill silhouette and photometric constraints (C)2011 IEEE. Reprinted, with permission, from Yusuke Yoshiyasu and Nobutoshi Yamazaki. Topology-adaptive multi-view photometric stereo. In IEEE Conference on Computer Vision and Pattern Recognition [2011]).

varying BRDFs similar to the approach by Goldman et al. 2005 — but in a multi-view setting. This approach even incorporates interreflection effects in the micro-structure and is related to BTF (Bidirectional Texture Function) acquisition techniques.

A disadvantage of approaches that project vertices into images is that the mesh resolution is not necessarily related to image resolution. Either a huge number of vertices has to be used or details in the images might be lost. Park et al. 2013 therefore propose a pipeline that uses mesh parametrization to define warpings from images onto a planar mesh representation. Thus, fine detail - in the form of a displacement map - can be recovered even with few mesh faces. The base mesh is obtained by merging multi-view stereo reconstructions from all views. Again, specular reflectance is treated as an outlier and the underlying photometric stereo technique is the one developed by Hayakawa 1994.

\section{Parametrized models}

Similar to $\S 5.2$ and $\S 7.2$, heavily model-based approaches can also be extended to multi-view settings. A very good example is provided by Yoon et al. [2010]. Their generative model consists of a finite number of distant light sources and shadow maps, the surface represented as a level set, reflectance parameters for the Blinn-Phong model (Blinn [1977]), and a set of pinhole cameras. This allows to render synthetic images of the scene given the current parameters. 
Visibility is handled by projecting the global surface into each image. Furthermore, their technique requires a model of the background scene to prevent the surface from shrinking to an empty set during the optimization. They also introduce an auxiliary normal field to decouple the appearance computation from the surface gradient for increased stability. The final optimization minimizes the error of all images compared to the renderings from the current parameters. It has to be performed in an alternating fashion - like in most other works - because of its complexity and the coupling of shape and reflectance in the image formation.

\section{Special setups}

Like single view photometric stereo the multi-view case often requires quite elaborate setups.

Lu and Little 1995] present a solution in a rather special setting: the light source and camera are co-located and the object spins around a known axis. They derive surface orientations pointing towards the light from intensity maxima and then exploit the known rotation angle of the object to track them. The tracked points allow the reconstruction of a one-dimensional slice of the BRDF. Once this is known, the surface orientation of all other surface points can be computed from its inverse.

Zhou et al. [2013] use a ring light system with 72 LEDs to capture 30 images under varying illumination for each camera position. This allows them to cope with arbitrary isotropic BRDFs by applying the technique of Alldrin and Kriegman 2007] in each view. The resulting iso-depth contours are then associated with sparse structure from motion points to obtain absolute depth values. The authors propagate depth along contours from each view to merge them into a globally consistent model. Once the geometry is acquired, a set of basis BRDFs and their mixing weights can be estimated because illumination is known from a calibration step.

Tunwattanapong et al. 2013] also require multiple light settings for each view. The incoming radiance distributions in such a sequence are shaped as spherical harmonics and created by an rotating arc of LEDs. This setup allows them to recover reflectance parameters in 
addition to surface orientation. The results from each camera position are then fused with a multi-view stereo reconstruction in a final surface optimization, which again alternates between several stages.

Less restrictive than these systems is the setup proposed by Paterson et al. [2005]. It consists of a calibration target and a flash light that is rigidly attached to the camera. They assume an almost planar target which allows them to establish pixel correspondences by warping the images. The recovery of normals and parameters of a modified Torrance-Sparrow BRDF model is alternated with a re-estimation of this warp.

Another technique that relies on a special capture setup to acquire series of images in each of multiple cameras is presented by Schuster [2010]. He proposes a photo-consistency measure based on the fitting error of a Cook Torrance BRDF at each hypothetical voxel and extracts a surface using graph cuts (Boykov and Kolmogorov 2003, Lempitsky and Boykov [2007]). As initialization, he constructs the visual hull to obtain visibility information and computes normals based on Helmholtz stereopsis. This requires the light and camera to swap positions, which is approximated in this case by a hemispherical dome of 151 cameras equipped with flash lights.

A similar setup, but augmented with several projectors for structured light acquisition, is used by Weinmann et al. [2012]. They acquire multi-camera, multi-light image sequences of an object rotating on a turntable. The surface is then extracted from an error function that combines structured light consistency and Helmholtz stereopsis into a single variational formulation.

Finally, the technique by Aliaga and $\mathrm{Xu}$ 2010 covers a whole range of single or multiple camera and projector setups. They combine Lambertian photometric stereo and structured light to recover oriented 3D points of the object and the geometric calibration of the system. Even for a single camera, they are able to formulate multi-view constraints on the depth of the points. That is possible by exploiting the fact that a projector can act as a virtual camera if pixel correspondences are established. 


\section{Over-complete representations}

We observe that several of the discussed multi-view photometric stereo methods (e.g. Joshi and Kriegman [2007, Hernandez et al. [2008], Yoshiyasu and Yamazaki [2011]) can be modeled by up to three general error terms

$$
E_{1}(X), \quad E_{2}(N), \quad E_{3}(X, N) .
$$

The first one is tied to multi-view constraints such as silhouettes. The second one finds optimal normals based on the current depth estimate. It is associated to photometric constraints, i.e. surface appearance. The third one updates the geometry based on these normal estimates. These terms are either combined into a sum or optimized one after the other.

Such a scheme uses a two-fold representation of the shape that includes a 3D proxy and an auxiliary normal field. It is an overcomplete representation since in the ideal case the normal field should be defined by the derivative of the geometry. This is sometimes described as a convenient formulation for optimization or as a necessity for stability, cf. Jin et al. [2004], Yoon et al. [2010]. It has the additional advantage to allow different resolutions in the two representations as exploited by Park et al. 2013. Often, the representations are coupled by a soft constraint in the spirit of $E_{3}=\|\partial X-N\|$. A popular method to perform that merging step is presented in the work of Nehab et al. 2005. In the future, a better understanding of the benefits and drawbacks of such an overcomplete formulation would be an interesting research topic. 


\section{Outside the lab}

The previous chapters show that many of the state of the art approaches draw on ideas and methods that have been developed ten or twenty years ago, e.g. matrix factorization or reflectance symmetries. We observe that today, more focus is put on integrating these ideas into ready to use systems and increasing their applicability through combination with other techniques. Two important aspects are the robustness with regard to non-ideal input images and the removal of requirements imposed on the capture setup. Thus, images downloaded from the Internet provide an interesting testbed for new developments. We emphasize this relevance by discussing some of the advances in computer vision related to Internet data $\$ 9.1$ and reconstructions under natural illumination $\$ 9.2$.

The scope of this chapter is slightly broadened and incorporates exemplary techniques from areas such as intrinsic image factorization or inverse rendering. This is justified because ideas from other fields on how to address some of the general challenges in uncontrolled settings, e.g. radiometric camera calibration, hopefully provide insights for photometric reconstructions as well. 


\subsection{Internet data}

\subsubsection{Image collections}

Snavely et al. 2006 show that it is possible to apply robust structure from motion to images from online photo sharing sites, e.g. Flickr. They recover the geometric camera parameters and a sparse set of $3 \mathrm{D}$ feature points. This allows a user to explore the scene by $3 \mathrm{D}$ browsing of the images and provides a novel way to communicate the impressions of one or several observers. Tompkin et al. 2012 extend 3D browsing to video sequences.

Since known camera parameters are a prerequisite for many reconstruction approaches, Snavely et al. also paved the way for works in that area. For example, Goesele et al. [2007] present a multi-view stereo approach for community photo collections. It relies heavily on the selection of suitable images to achieve robustness against scale differences or occluding clutter. Furukawa and Ponce [2010] achieve robustness through several filter steps that reason about visibility and consistency of $3 \mathrm{D}$ patches. These approaches recover the full scene geometry instead of a sparse feature set and can convey the 3D impression of the scene even better. Goesele et al. 2010. exploit this as proxy geometry in an image-based rendering system to achieve a more convincing browsing experience in the presence of unreliable data. Agarwal et al. 2009 and Frahm et al. 2010 extend the reconstruction approaches to whole city areas using clustering techniques.

These techniques focus on the reconstruction of the 3D geometry and try to be robust against appearance changes. Such changes, e.g. due to varying illumination or view point, are at the heart of photometric techniques and convey additional information. Garg et al. 2009] extend results from Belhumeur and Kriegman 1998 about the space of possible images for a given scene to photo collections. They project all images onto a 3D model and factorize the matrix that arises from stacking intensities at each vertex. The factorization can be interpreted as basis images for the scene that capture variation along different axes. While these axes often correspond to a certain meaning, e.g. mean image and shading variations in the Lambertian case, there are no clear 
semantics for decompositions in more complex scenes.

A more usable separation of appearance for uncontrolled scenes is achieved by Haber et al. 2009. They build on a 3D model as produced by Goesele et al. 2007] and recover the reflectance - encoded as mixing weights per surface point with respect to a selection of Cook-Torrance BRDFs - and illumination $L_{s, i}$ in each view of an image collection. A linearization

$$
\begin{aligned}
\rho & =\sum_{k} \rho_{k} \Psi_{k}, \\
L_{s, i} & =\sum_{l} L_{s, i, l} \Psi_{l}
\end{aligned}
$$

based on Haar-Wavelets lets them formulate the outgoing radiance as

$$
L_{i}=\sum_{k, l} \rho_{k} L_{s, i, l} \underbrace{\int \Psi_{k}(\omega) \Psi_{l}(\omega) \mathrm{d} \omega}_{=: B_{k, l}}=\left(\rho_{1}, \ldots\right) \cdot B \cdot\left(\begin{array}{c}
L_{s, i, 1} \\
\vdots
\end{array}\right)
$$

The final optimization over all surface points $X_{j}$ and images

$$
\arg \min \sum_{j, i}\left(I_{i}\left(X_{j}\right)-L_{i}\left(X_{j}\right)\right)^{2}
$$

alternates between both unknowns $\rho, L_{s}$ and yields a decomposition that gives plausible relighting results. Figure 9.1 compares the rendering results to one of the input images which was not used during reconstruction.

Diaz and Sturm 2011 also use a 3D model to estimate illumination, but additionally consider the camera response function. They represent this function as a linear combination of the EMOR basis (Empirical Model of Response; Grossberg and Nayar 2003], Grossberg and Nayar. 2004])

$$
f=h_{0}+\sum_{i=1, \ldots} \alpha_{i} h_{i}
$$

and include the coefficients $\alpha_{i}$ in the lighting optimization. While this work recovers only the diffuse albedo, it is one of the few that actually take non-linear response curves beyond a straightforward gamma correction into account. 

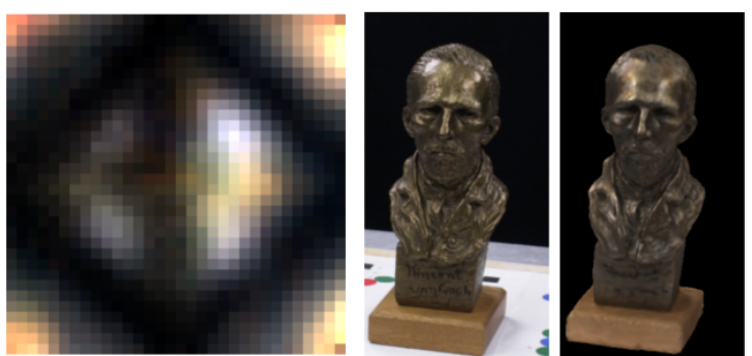

Figure 9.1: Haber et al. 2009 first recover the geometry, e.g. using multi-view stereo, and then estimate an environment map (left) and the reflectance properties. Combining these constituents, they achieve synthetic renderings (right) that are quite similar to the actually observed image intensities (middle). This approach works even for uncontrolled image collections as found on the Internet and can be used for relighting or appearance editing (C)2009 IEEE. Reprinted, with permission, from Tom Haber, Christian Fuchs, Philippe Bekaert, Hans-Peter Seidel, Michael Goesele, and Hendrik P.A. Lensch. Relighting objects from image collections. In IEEE Conference on Computer Vision and Pattern Recognition [2009], pages 627$634)$.

\subsubsection{Webcam data}

Another growing source of Internet data are video sequences, e.g. from webcams. Jacobs et al. 2007a collect images from hundreds of webcams and discover that the coordinates of a principal component analysis (PCA) for different cameras behave similarly over time. This allows them to define a canonical basis and assign pixel labels with a common meaning in all cameras. In subsequent works, Jacobs et al. 2007b, 2010, 2013a b are able to estimate the location of an unknown camera by comparing PCA coordinates with those of known webcams. The depth of individual scene points can be recovered from correlations induced by cloud shadows. Additionally, the movement of the clouds themselves yields cues to predict vanishing points and thus calibrate the camera geometrically. Lalonde et al. [2008, 2010] demonstrate how the sun and sky illumination can be exploited for such a calibration as well. They relate azimuth and zenith angles in a geo-reference coordinate system to pixel coordinates in the camera. Inserting this transformation into the analytical sky model presented by Perez et al. [1993] yields a function that predicts radiance for sky pixels. The camera calibration is 
then found by an optimization that compares the predicted radiance values to observed pixel intensities.

Apart from calibration and geo-location, webcams are also used to decompose scene appearance and enable editing of its components. Sunkavalli et al. 2007) analyze the intensity profiles of pixels in dense video to identify transitions from shadow to sunlight. They find that profiles within a scene are similar up to scale and a shift in time. This allows them to define time-varying basis curves for the sun and sky contribution. The corresponding coefficients with respect to this basis are recovered similar to Lawrence et al. 2006 and constitute a decomposition into ambient light, albedo, and sun reflectance. Sunkavalli et al. 2008 additionally recover partial surface normals - projected on the plane of solar movement-based on color changes. Lalonde et al. 2009 estimate the sky appearance and sun visibility in each frame for a collection of webcams. These cues are used to match the illumination conditions between different cameras and thus enable the transfer of correctly lit objects. The authors also insert synthethic objects by illuminating them according to the parameters of the underlying sky model in each image.

\subsubsection{Recovering surface orientation}

Only few approaches focus on surface orientation as an important part of scene appearance. Shen and Tan [2009] use a decomposition of illumination as proposed by Basri and Jacobs 2001b to estimate weather conditions in Internet photo collections. This also includes computation of surface normals, but only at sparse feature points that have been matched between images. Their approach relies on a selection of suitable features with sufficiently Lambertian behavior and support in close-by views.

Ackermann et al. 2012 aim for a more complete decomposition of scene appearance. They rely on a fixed camera position as provided by Internet webcams. At each pixel $j$, the direct sun light interacts with a mixture of basis materials $\rho_{k}$ modulated by a binary shadow function $\xi$. The sky is modeled by a per-pixel additive contribution $\beta$ which gives 

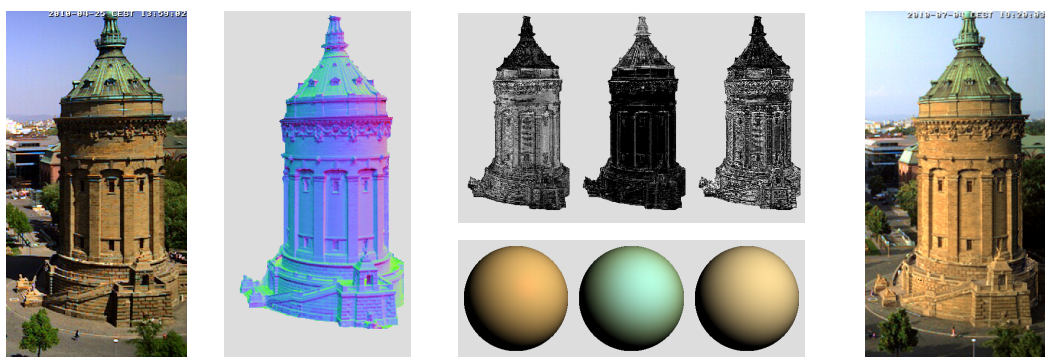

Figure 9.2: Exemplary results for the webcam-based reconstruction by Ackermann et al. 2012]. Left to right: One of the input images, the recovered normal map, three basis BRDFs below their respective material maps, and a synthetic rendering for a novel sun position (C)2012 IEEE. Reprinted, with permission, from Jens Ackermann, Fabian Langguth, Simon Fuhrmann, and Michael Goesele. Photometric stereo for outdoor webcams. In IEEE Conference on Computer Vision and Pattern Recognition [2012], pages 262-269).

an overall radiance of

$$
L_{i, j}=l_{i}\left(\sum_{k} \gamma_{j, k} \rho_{k}\left(n_{j}, \omega_{i}\right) \xi_{i, j}+\beta_{j}\right)
$$

for relative sun intensity $l_{i}$ and sun direction $\omega_{i}$. The non-linear dependence within this large set of parameters, including normals $n_{j}$, yields a complex optimization problem. The authors decompose it into three steps that each keep some of the variables fixed. The final result is shown in Figure 9.2 and yields a decomposition that can be used for relighting. This work relies heavily on a preprocessing pipeline that selects suitable images from a webcam stream and calibrates the camera.

In a similar setting, Abrams et al. 2012] recover dense surface normals, albedo, and the radiometric camera calibration from outdoor webcams. They exploit the known sun position with respect to a georeferenced coordinate system in order to arrive at a traditional photometric stereo problem for Lambertian surfaces. This task becomes more complex through an ambient term and the non-linear response, which is represented as a combination of EMOR (Grossberg and Nayar [2003, Grossberg and Nayar. 2004]) basis curves. Abrams et al. 2012 split the corresponding optimization into two alternating steps and approximate each of them with a linear subproblem which can be solved efficiently. This allows them to use hundreds of images compared to 
the careful selection of about 50 images by Ackermann et al. [2012]. The increase in data gives better robustness to outliers that are not captured by their image formation model.

More recently, Shan et al. 2013 have applied a related outdoor lighting model for photometric reconstruction on community photo collections. They first recover a mesh using a pipeline of structure from motion and multi-view stereo as described in \$9.1.1. Then, they estimate the sun direction and sky contributions in each image and finally compute the diffuse albedo and normal at each vertex in a non-linear optimization. The evaluation is performed in the form of a so called Visual Turing Test, i.e. renderings and original images are presented to humans who have to specify which one is more realistic. Several low resolution images actually pass that test. Visual artifacts and not reconstructed content such as people, however, reveal the limitations of current approaches towards relightable appearance reconstruction on Internet data.

Finally, Jung et al. [2015] perform photometric stereo using a handful of outdoor images of a scene captured on a single sunny day by a static camera. Their key observation is that modelling the complete sky as light source (using a variant of an analytic sky model by Preetham et al. 1999]) allows to overcome the restriction that the movement of the sun on a single day contains not enough variance to recover full normals. Using their analytic sky model and a set of 1000 normal hypotheses, they they can compute 1000 corresponding radiance profile candidates per pixel (up to a scaling factor), and compute their correlation with the observed values in the images captured at different times of day. Per-pixel surface normals are then assigned using an MRF-based optimization that provides regularization and allows to fill holes at pixels that received too few valid observations.

\subsection{Natural illumination}

Compared to traditional approaches that require laboratory conditions, Internet images are at least two levels more challenging. It makes sense to also think about in-between settings, e.g. a controlled outdoor 
dataset, which are not yet solved entirely.

Sato and Ikeuchi 1994a, 1995 apply their separation of diffuse and specular components based on illuminant color (Sato and Ikeuchi [1994b] to outdoor images under clear skies. They normalize image regions to simulate a uniform albedo and obtain its value from the brightest pixels in the image sequence. Then, the angle between the normal and the solar plane is obtained at each pixel simply as its maximal intensity divided by the albedo. Narasimhan et al. [2002] study appearance variation due to weather changes in long sequences of controlled outdoor data. Atmospheric scattering which depends on the distance of the scene point from the camera allows them to compute approximate depth for each pixel.

Hung et al. 2015] capture the environmental illumination from a mirror sphere. Assuming a calibrated camera, they discretize the rendering equation as

$$
I=\sum_{j} \rho c_{j}\left\langle n, \omega_{j}\right\rangle
$$

where $c_{j}$ is the light intensity from direction $\omega_{j}$. For each surface point, they compute the pair $(n, \rho)$ that best explains the observations $I_{i}$ in multiple images according to Equation 9.7. These ideas are closely related to the work by $\mathrm{Yu}$ et al. 2013], who also use a mirror sphere and discretize the illumination into a set of point light sources. They assume a Lambertian reflectance, treat specularities as outliers, and employ a simple heuristic to estimate self occlusion. Their results on outdoor images expose problems due to insufficient variation of light directions. This might be one of the reasons why most current work on outdoor reconstructions, e.g. Oxholm and Nishino [2014], is focused on fixed illumination settings. Especially techniques that decompose the illumination and reflectance in images of a known shape, such as presented by Dong et al. 2014b, show some promising advances.

If the whole light field of the scene is known, it can be decomposed into a basis of spherical harmonics. Ramamoorthi and Hanrahan 2001 show that those frequencies of illumination and reflectance that are also present in the outgoing light field - which they interpret as a spherical convolution - can be recovered from its coefficients. This task is much harder if only parts of the light field, e.g. a single image, is available 


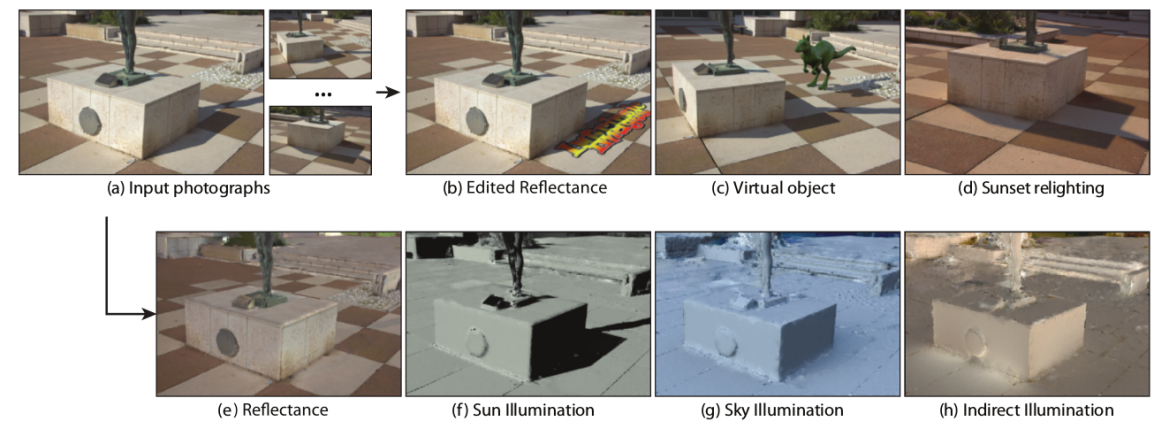

Figure 9.3: Laffont et al. 2013 recover the geometry from multiple images $(a)$ and estimate illumination parameters. Photographs are split into the reflectance $(e)$ and the contributions due to sun $(f)$, sky $(g)$, and indirect $(h)$ illumination. Various editing operations $(b-d)$ can be based on such a decomposition (c)2013 IEEE. Reprinted, with permission, from Pierre-Yves Laffont, Adrien Bousseau, and George Drettakis. Rich intrinsic image decomposition of outdoor scenes from multiple views. IEEE Transactions on Visualization and Computer Graphics, 19:210-224, 2013).

and requires additional constraints or regularizing assumptions. For example, any image of a sphere can be explained not only by the true illumination and BRDF, but also by a perfect mirror illuminated with that image.

Experiments conducted by Fleming et al. 2003 indicate that humans are able to match reflectance properties independent of illumination and that they do so based on prior knowledge about the behavior of natural illumination. Romeiro and Zickler 2010a therefore exploit the statistics of natural illumination to define a prior on the possible lighting $L_{s}$. Their idea is to marginalize the posterior $p\left(L_{s}, R \mid I\right) \propto p\left(I \mid L_{s}, R\right) p(R) p\left(L_{s}\right)$ over the lighting to obtain the probability $p(R \mid I)$ of the reflectance $R$ given an image $I$. Computing the mean of this distribution amounts to selecting a reflectance that not only explains the image for a single illumination, but for all illuminations - according to their probability. Lombardi and Nishino 2012 also consider objects of known shape but additionally recover illumination explicitly.

Laffont et al. 2012] reconstruct a point cloud of a scene using multiview stereo. This serves as proxy to compute the contributions of the 
sun, sky, and indirect lighting based on a measured environment map. Once sun visibility and reflectance are computed for each 3D point, all this information is projected into the image and propagated over all pixels. Laffont et al. 2013 compute an intrinsic image decomposition but allow for varying illumination between images, which makes the technique applicable to Internet photo collections. They observe that one of the main problems with that kind of data is the unknown camera response. Figure 9.3 lists the components of their decomposition. Lee et al. 2012 also decompose multiple images but do so for a video stream with fixed illumination. Again, their algorithm is supported by a priori known shape information-acquired from a depth camera. 


\section{0}

\section{Conclusion}

In this chapter, we first summarize in \$10.1 the core ideas of modern photometric stereo techniques following the main structure of the review. We show how approaches can be judged by their generality with respect to lighting, reflectance and viewpoint distribution as well as by their robustness with respect to different real-world effects. We then discuss future research directions for photometric stereo and related approaches in $\$ 10.2$ and conclude in $\$ 10.3$ with thoughts on the future use of photometric stereo.

\subsection{Summary}

(Lambertian) photometric stereo is a simple yet very powerful technique to reconstruct a surface from a couple of images taking under varying illumination. Its essence can be captured in a handful of formulae as described at the beginning of Chapter 2. Despite its simplicity, photometric stereo yields high quality reconstructions if the scene and the capture setup conform to the basic assumptions underlying the technique. As described in Chapter 3 most of the fundamental concepts of photometric stereo were developed in seminal works during 


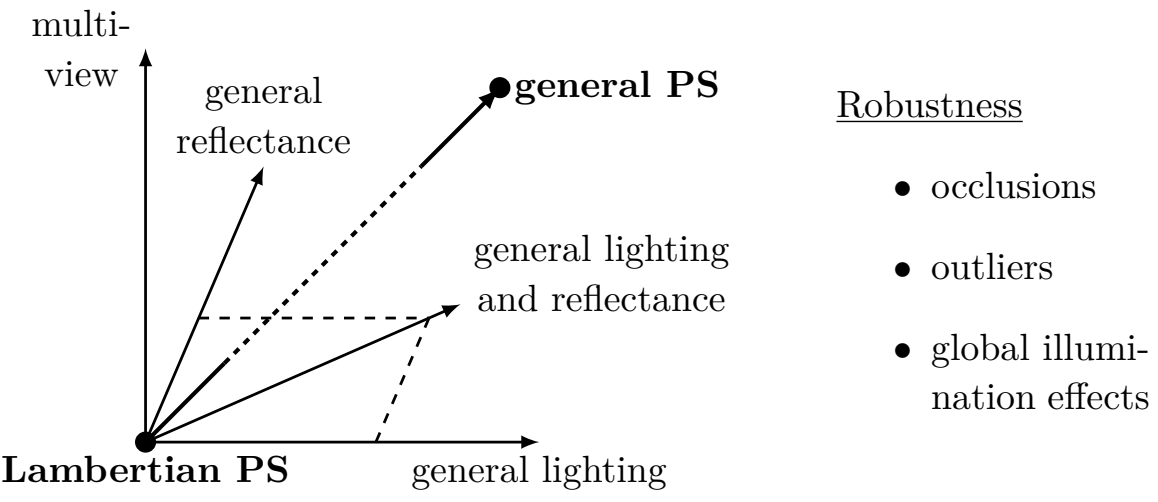

Figure 10.1: An overview over challenges in photometric stereo. The left half of the figure shows the progression from basic Lambertian photometric stereo as introduced in the 1980s towards the solution of the general photometric stereo problem. The right half of the figure lists issues that can be addressed using robustness in the reconstruction steps.

the 1980s. Most of its limitations and shortcomings were also already known at that time. These include, e.g. the need for specific capture settings and calibrated capture equipment as well as the fact that outliers such as shadows or surface interreflections need to be treated very carefully. Most of the research in the last decades focussed on adressing these restrictions in order to generalize photometric stereo to less constrained and less controlled settings. One could therefore ask the question: "When will photometric stereo be solved?"

One answer to this question is that photometric stereo is solved once we can take any arbitrary set of images of an object or scene with large enough input variation and reconstruct a perfect model of its surface orientation from these images. This problem can be decomposed into two almost independent issues - on one hand handling general lighting, reflectance and viewpoints and on the other hand being robust to various effects. We will in the following categorize and discuss these using Figure 10.1 as visual aid.

Research towards general lighting lead to uncalibrated photometric stereo algorithms that are oblivious to the lighting setup. A recurring method in this context is a low-rank matrix factorization as introduced 
in Chapter 4. Simultaneously, research has advanced towards removing the need to know the reflectance properties of the target object. General invariants of the BRDF or reflection geometry define constraints that are exploited by the techniques in Chapter 5 . Any algorithm in these two fields is based on assumptions and these might not be fulfilled in practice. Whether this presents a serious problem depends on the specific application. One way to handle them is to use robust methods. Chapter 6 addresses this using techniques for individual error sources and methods that are robust to outliers in general.

Combining the concepts introduced in Chapters 4 6 allows to tackle reconstruction tasks where both, lighting and reflectance, are unknown. One solution to this problem is to model every aspect of light transport explicitly. In addition, Chapter 7 discusses works that exploit orientation consistency and appearance profiles. These techniques are independent of a parametric BRDF or lighting model but require typically a large amount of input data.

Chapter 8 adds a new perspective by considering the camera position as an additional variable. In this chapter, we collect multiview techniques regardless of their assumptions about illumination and BRDF which leads to a broad variety of approaches. Again, some ideas from the single-view setting can be transferred, e.g. heavily parametrized models, while others are newly introduced such as reconstruction by deformation of an initial proxy mesh.

All these works demonstrate how far the field of photometric reconstructions has evolved since its beginnings. Even very general algorithms in terms of input data are, however, still restricted to capture under controlled settings such as a laboratory. Missing robustness, requirements on the capture setup, or an implicitly assumed camera calibration reduce their applicability. Related fields in computer vision have seen a definite shift towards uncontrolled scenarios and applications in consumer products. Chapter 9 discusses these advances and some of the first approaches to exploit photometric cues in natural environments and even on Internet data. 


\subsection{Discussion and outlook}

We now discuss various aspects that are (in our subjective opinion) important for the future development of photometric stereo.

\subsubsection{Broadening the applicability}

The advances in generalizing photometric stereo with respect to unknown light or reflectance have revealed valuable insights during the last decades. We believe, however, that significant advancements will in the future most likely not be made along a single of these axes. Instead, it seems more promising to make photometric techniques ready for applications outside the lab. The approaches for general lighting and reflectance in Chapter 7 provide a starting point towards that end. But to achieve a noticeable increase in applicability a greater focus on uncontrolled settings and real-world capture conditions, e.g. a consumer camera with an attached flash unit, is required.

Another trend that can be deduced from Chapter 8 is the development of multi-view techniques. Varying camera positions are also an important step towards less controlled scenarios. Currently, most of these techniques are, however, not as general in the assumed lighting model as those in Chapter 7. Furthermore, the applicability of photometric reconstruction would benefit from research towards better radiometric auto-calibration methods. Images taken with a consumer camera by a casual user are meant (and made by the camera) to look good not to be well suited for reconstruction.

Finally, we see the transition from reconstructing single target objects to complete scenes, e.g. a room, as a worth-while advancement. While some methods, e.g. voxel-based multi-view approaches, should be able to handle that case in theory, they are traditionally evaluated on single objects only. Multiple objects introduce many discontinuities in geometry and appearance which pose novel and interesting challenges.

\subsubsection{How general do we have to be?}

Many researchers would agree that bringing the image creation models used for photometric stereo closer to reality, e.g. by incorporating 
interreflections, while still being able to invert them or to find suitable invariants is a worthwhile goal. It remains, however, unclear how well the model's assumptions must match reality in order to achieve good results over a wide range of objects. For example, some techniques relying on a Lambertian reflectance model have shown success even when applied to specular materials by treating specular reflections as outliers. Still, a drawback of very general methods might be that they are outperformed by more specialized techniques, which can exploit prior knowledge in their respective target areas.

Another question is how to find the best balance between building explicit models and using more data-driven approaches. For example, appearance profiles do not depend on a strict lighting model but might carry not enough information if only a few input images are available. Parametric models can be a way to work around a lack of input data by enforcing stricter constraints at the cost of reduced flexibility.

Finally, no matter how sophisticated the theory underlying an approach is it will never completely match reality. There will always be a case where observations do not follow all assumptions made by the algorithm. These outliers can misguide the technique to a wrong solution if they are not properly handled, e.g. using ideas from $\$ 6.2$. The challenge is to find the balance between sufficient robustness and accidentally throwing away too much information.

\subsubsection{Combining different techniques}

It is highly unlikely that a single algorithm or key idea will be widely or even universally applicable. Even for different techniques from the same general class, e.g. uncalibrated photometric stereo, it often depends on the specific scene which one technique performs best. Thus, combinations of several approaches with complementary advantages and disadvantages seem like a promising direction. For example, the boundaries between purely texture-based stereo approaches and photometric techniques already vanish as discussed in Chapter 8 . Another strategy could be to use a different algorithm for different parts of a scene or depending on the object type. Then, the interesting challenge would be to choose the best suited technique automatically. 


\subsubsection{Data issues}

In general, the usefulness of input images is strongly related to the observable shading variations. If the shading does not change, e.g. because the illumination remains fixed with respect to the surface, there is no chance of success for any photometric approach (unless shape from shading techniques with strong assumptions on the surface properties can be employed). This might be one of the reasons why everyday scenarios are rarely considered by photometric stereo methods. Images captured in an office or outdoors will not display sufficient lighting variation unless taken at different times of day. Such issues also arise in a lab setting, but achieving consistent results over a large range of capture conditions and target objects becomes much harder for uncontrolled scenarios.

The opposite of missing input data occurs for some of the approaches in Chapter 9. On Internet images, e.g. months or even years of webcam videos, the vast amount of data would cause many traditional techniques to reach the limits of computational resources such as memory or processing time. It is therefore crucial to reduce the amount of input data by selecting suitable images for reconstruction. Selection strategies not only ensure the computational feasibility but can also increase robustness by removing non-ideal, e.g. under-exposed, images. Estimating the value of an image for the overall reconstruction is not yet considered by most approaches. Instead, they typically assume that all images are equally important and beneficial.

\subsection{Looking ahead}

Photometric stereo, which can be simply summarized as a way to reconstruct surface orientation from a set of images with varying illumination, has historically been a very important technique. Its core ideas can be applied to a wide variety of domains including surface reconstruction, rendering, relighting as well as industrial inspection. The transition towards more general algorithms and less controlled capture conditions will make it ready for the demands of future application scenarios. We are thus very confident that photometric stereo will also 
play a crucial role in the upcoming years and decades, most likely as an integral part of comprehensive and versatile reconstruction systems. 


\section{Acknowledgements}

We would like to thank Reinhard Klein for his valuable advice and suggestions, including the suggestion to publish this review. We also thank Mate Beljan, Simon Fuhrmann, Stepan Konrad, Arjan Kuijper, Fabian Langguth, Nils Moehrle, Martin Ritz and Andre Stork for their contributions to individual parts of this review. Special thanks go to the various authors of related works that gave us kind permission to use some of their figures and images. Furthermore, we would like to acknowledge the valuable input and constructive criticism provided by the reviewers. We also thank the editor Brian Curless and the publisher James Finlay for their support during the production of this review. Last but not least, we thank our families and friends who strongly supported us on our sometimes difficult path towards writing this review.

This work was supported in part by the European Commission's Seventh Framework Programme under grant agreements no. ICT611089 (CR-PLAY), as well as the DFG Emmy Noether fellowship GO 1752/3-1. 


\section{References}

Austin Abrams, Christopher Hawley, and Robert Pless. Heliometric stereo: Shape from sun position. In European Conference on Computer Vision, pages 357-370, 2012.

Jens Ackermann, Martin Ritz, Andre Storck, and Michael Goesele. Removing the example from example-based photometric stereo. In European Conference on Computer Vision Workshops, Lecture Notes in Computer Science, pages 197-210, 2010.

Jens Ackermann, Fabian Langguth, Simon Fuhrmann, and Michael Goesele. Photometric stereo for outdoor webcams. In IEEE Conference on Computer Vision and Pattern Recognition, pages 262-269, 2012.

Jens Ackermann, Simon Fuhrmann, and Michael Goesele. Geometric point light source calibration. In Proceedings of the Vision, Modeling, and Visualization Workshop, pages 161-168, 2013.

Jens Ackermann, Fabian Langguth, Simon Fuhrmann, Arjan Kuijper, and Michael Goesele. Multi-view photometric stereo by example. In International Conference on 3D Vision, 2014.

Sameer Agarwal, Noah Snavely, Ian Simon, Steven M. Seitz, and Richard Szeliski. Building Rome in a day. In IEEE International Conference on Computer Vision, pages 72-79, 2009.

Amit K. Agrawal, Ramesh Raskar, and Rama Chellappa. What is the range of surface reconstructions from a gradient field? In European Conference on Computer Vision, pages 578-591, 2006. 
Naveed Ahmed, Christian Theobalt, Petar Dobrev, Hans-Peter Seidel, and Sebastian Thrun. Robust fusion of dynamic shape and normal capture for high-quality reconstruction of time-varying geometry. In IEEE Conference on Computer Vision and Pattern Recognition, 2008.

Miika Aittala, Tim Weyrich, and Jaakko Lehtinen. Practical SVBRDF capture in the frequency domain. ACM Transactions on Graphics, 32:110:1110:12, 2013.

Daniel G. Aliaga and Yi Xu. A self-calibrating method for photogeometric acquisition of 3D objects. Pattern Analysis and Machine Intelligence, IEEE Transactions on, 32(4):747-754, April 2010. ISSN 0162-8828.

Neil G. Alldrin and David J. Kriegman. Toward reconstructing surfaces with arbitrary isotropic reflectance: A stratified photometric stereo approach. In IEEE International Conference on Computer Vision, pages 1-8, 2007.

Neil G. Alldrin, Satya P. Mallick, and David J. Kriegman. Resolving the generalized bas-relief ambiguity by entropy minimization. In IEEE Conference on Computer Vision and Pattern Recognition, 2007.

Neil G. Alldrin, Todd E. Zickler, and David J. Kriegman. Photometric stereo with non-parametric and spatially-varying reflectance. In IEEE Conference on Computer Vision and Pattern Recognition, 2008.

Takahito Aoto, Takafumi Taketomi, Tomokazu Sato, Yasuhiro Mukaigawa, and Naokazu Yokoya. Position estimation of near point light sources using a clear hollow sphere. In International Conference on Pattern Recognition, pages 3721-3724, 2012.

Jonathan T. Barron and Jitendra Malik. Shape, albedo, and illumination from a single image of an unknown object. In IEEE Conference on Computer Vision and Pattern Recognition, 2012.

Jonathan T. Barron and Jitendra Malik. Intrinsic scene properties from a single RGB-D image. In IEEE Conference on Computer Vision and Pattern Recognition, 2013.

Svetlana Barsky and Maria Petrou. The 4-source photometric stereo technique for three-dimensional surfaces in the presence of highlights and shadows. IEEE Transactions on Pattern Analysis and Machine Intelligence, 25(10): 1239-1252, Oct 2003. ISSN 0162-8828.

Ronen Basri and David Jacobs. Lambertian reflectance and linear subspaces. In IEEE International Conference on Computer Vision, 2001a.

Ronen Basri and David Jacobs. Photometric stereo with general, unknown lighting. In IEEE Conference on Computer Vision and Pattern Recognition, 2001b. 
Peter N. Belhumeur and David J. Kriegman. What is the set of images of an object under all possible illumination conditions? International Journal of Computer Vision, 28:245-260, 1998.

Peter N. Belhumeur, David J. Kriegman, and Alan L. Yuille. The bas-relief ambiguity. International Journal of Computer Vision, 35:33-44, 1999.

Mate Beljan, Jens Ackermann, and Michael Goesele. Consensus multi-view photometric stereo. In DAGM/OAGM Symposium, Lecture Notes in Computer Science, pages 287-296, 2012.

Dimitri P. Bertsekas and Werner Rheinboldt. Constrained Optimization and Lagrange Multiplier Methods. Computer science and applied mathematics. Elsevier Science, 2014.

Anak Bhandari, Børge Hamre, Øvynd Frette, Lu Zhao, Jakob J. Stamnes, and Morten Kildemo. Bidirectional reflectance distribution function of spectralon white reflectance standard illuminated by incoherent unpolarized and plane-polarized light. Applied Optics, 50(16):2431-2442, Jun 2011.

Neil Birkbeck, Dana Cobzas, Martin Jagersand, and Peter Sturm. Variational shape and reflectance estimation under changing light and viewpoints. In European Conference on Computer Vision, 2006.

James F. Blinn. Models of light reflection for computer synthesized pictures. SIGGRAPH Computer Graphics, 11:192-198, 1977.

Dorothea Blostein and Narendra Ahuja. Shape from texture: Integrating texutre-element extraction and surface estimation. IEEE Transactions on Pattern Analysis and Machine Intelligence, 11:1233-1251, 1989.

Thomas Bonfort and Peter Sturm. Voxel carving for specular surfaces. In IEEE International Conference on Computer Vision, 2003.

Yuri Boykov and Vladimir Kolmogorov. Computing geodesics and minimal surfaces via graph cuts. In IEEE International Conference on Computer Vision, pages 26-33, 2003.

Peter Burt and Bela Julesz. A disparity gradient limit for binocular fusion. Science, 208:615-617, 1980.

Rodrigo L. Carceroni and Kiriakos N. Kutulakos. Multi-view scene capture by surfel sampling: From video streams to non-rigid 3D motion, shape \& reflectance. In IEEE International Conference on Computer Vision, 2001.

Manmohan Krishna Chandraker, Fredrik Kahl, and David J. Kriegman. Reflection on the generalized bas-relief ambiguity. In IEEE Conference on Computer Vision and Pattern Recognition, 2005. 
Manmohan Krishna Chandraker, Sameer Agarwal, and David Kriegman. Shadowcuts: Photometric stereo with shadows. IEEE Conference on Computer Vision and Pattern Recognition, 0:1-8, 2007.

Manmohan Krishna Chandraker, Jiamin Bai, and Ravi Ramamoorthi. A theory of differential photometric stereo for unknown BRDFs. In IEEE Conference on Computer Vision and Pattern Recognition, 2011.

Manmohan Krishna Chandraker, Dikpal Reddy, Yizhou Wang, and Ravi Ramamoorthi. What object motion reveals about shape with unknown BRDF and lighting. In IEEE Conference on Computer Vision and Pattern Recognition, 2013.

Tongbo Chen, Michael Goesele, and Hans-Peter Seidel. Mesostructure from specularity. In IEEE Conference on Computer Vision and Pattern Recognition, 2006.

E. North Coleman and Ramesh Jain. Obtaining 3-dimensional shape of textured and specular surfaces using four-source photometry. Computer Graphics and Image Processing, 18:309-328, 1982.

Toby Collins and Adrien Bartoli. 3D reconstruction in laparoscopy with closerange photometric stereo. In International Conference on Medical Image Computing and Computer Assisted Intervention, pages 634-642, 2012.

M. Daum and G. Dudek. On 3-D surface reconstruction using shape from shadows. In IEEE Conference on Computer Vision and Pattern Recognition, 1998.

James E. Davis, Ruigang Yang, and Liang Wang. BRDF invariant stereo using light transport constancy. In IEEE International Conference on Computer Vision, pages 436-443, 2005.

Paul Debevec and Jitendra Malik. Recovering high dynamic range radiance maps from photographs. In Proceedings of ACM SIGGRAPH, 1997.

Paul Debevec, Tim Hawkins, Chris Tchou, Haarm-Pieter Duiker, Westley Sarokin, and Mark Sagar. Acquiring the reflectance field of a human face. In Proceedings of ACM SIGGRAPH, 2000.

Amael Delaunoy, Emmanuel Prados, and Peter N. Belhumeur. Towards full 3D Helmholtz stereovision algorithms. In 12th Asian Conference on Computer Vision, 2010.

Mauricio Diaz and Peter Sturm. Exploiting image collections for recovering photometric properties. In Computer Analysis of Images and Patterns, 2011. 
Bo Dong, Kathleen D. Moore, Weiyi Zhang, and P. Peers. Scattering parameters and surface normals from homogeneous translucent materials using photometric stereo. In IEEE Conference on Computer Vision and Pattern Recognition, pages 4321-4328, 2014a.

Yue Dong, Guojun Chen, Pieter Peers, Jiawan Zhang, and Xin Tong. Appearance-from-motion: Recovering spatially varying surface reflectance under unknown lighting. ACM Transactions on Graphics, 33:193:1-193:12, 2014b.

Richard Dosselmann and Xue Dong Yang. Improved method of finding the illuminant direction of a sphere. SPIE Journal of Electronic Imaging, 22, 2013.

Ondrej Drbohlav and Mike Chantler. Can two specular pixels calibrate photometric stereo? In Proceedings of the 10th IEEE International Conference on Computer Vision - Volume 2, ICCV '05, pages 1850-1857, Washington, DC, USA, 2005. IEEE Computer Society. ISBN 0-7695-2334-X-02.

Ondrej Drbohlav and Radim Sára. Specularities reduce ambiguity of uncalibrated photometric stereo. In Proceedings of the 7th European Conference on Computer Vision-Part II, ECCV '02, pages 46-62, London, UK, UK, 2002. Springer-Verlag. ISBN 3-540-43744-4.

Jean-Denis Durou, Jean-François Aujol, and Frédéric Courteille. Integrating the normal field of a surface in the presence of discontinuities. In Energy Minimization Methods in Computer Vision and Pattern Recognition, pages 261-273, 2009.

Anders Eriksson and Anton van den Hengel. Efficient computation of robust low-rank matrix approximations in the presence of missing data using the L1 norm. In IEEE Conference on Computer Vision and Pattern Recognition, 2010.

European Machine Vision Association. Standard for characterization of image sensors and cameras, 2010.

Paolo Favaro and Thoma Papadhimitri. A closed-form solution to uncalibrated photometric stereo via diffuse maxima. In IEEE Conference on Computer Vision and Pattern Recognition, 2012.

Roland W. Fleming, Ron O. Dror, and Edward H. Adelson. Real-world illumination and the perception of surface reflectance properties. Journal of Vision, 3:347-368, 2003.

David A. Forsyth and Jean Ponce. Computer Vision: A Modern Approach. Prentice Hall Professional Technical Reference, 2011. 
Jan-Michael Frahm, Pierre Fite-Georgel, David Gallup, Tim Johnson, Rahul Raguram, Changchang Wu, Yi-Hung Jen, Enrique Dunn, Brian Clipp, Svetlana Lazebnik, and Marc Pollefeys. Building Rome on a cloudless day. In European Conference on Computer Vision, 2010.

Robert T. Frankot and Rama Chellappa. A method for enforcing integrability in shape from shading algorithms. IEEE Transactions on Pattern Analysis and Machine Intelligence, 10:439-451, 1988.

Yasutaka Furukawa and Jean Ponce. Accurate, dense, and robust multiview stereopsis. IEEE Transactions on Pattern Analysis and Machine Intelligence, 32:1362-1376, 2010.

Rahul Garg, Hao Du, Steven M. Seitz, and Noah Snavely. The dimensionality of scene appearance. In IEEE International Conference on Computer Vision, 2009.

Athinodoros S. Georghiades. Incorporating the Torrance and Sparrow model of reflectance in uncalibrated photometric stereo. In IEEE International Conference on Computer Vision, 2003.

Abhijeet Ghosh, Graham Fyffe, Borom Tunwattanapong, Jay Busch, Xueming $\mathrm{Yu}$, and Paul Debevec. Multiview face capture using polarized spherical gradient illumination. ACM Transactions on Graphics, 30:129:1-129:10, 2011.

Michael Goesele, Noah Snavely, Brian Curless, Hugues Hoppe, and Steven M. Seitz. Multi-view stereo for community photo collections. In IEEE International Conference on Computer Vision, 2007.

Michael Goesele, Jens Ackermann, Simon Fuhrmann, Carsten Haubold, Ronny Klowsky, Drew Steedly, and Richard Szeliski. Ambient point clouds for view interpolation. ACM Transactions on Graphics, 29:95:1-95:6, 2010.

Dan Goldman, Brian Curless, Aaron Hertzmann, and Steven M. Seitz. Shape and spatially-varying BRDFs from photometric stereo. In IEEE International Conference on Computer Vision, pages 341-348, 2005.

Cindy M. Goral, Kenneth E. Torrance, Donald P. Greenberg, and Bennett Battaile. Modeling the interaction of light between diffuse surfaces. In Proceedings of ACM SIGGRAPH, 1984.

Michael D. Grossberg and Shree K. Nayar. What is the space of camera response functions? In IEEE Conference on Computer Vision and Pattern Recognition, 2003.

Michael D. Grossberg and Shree K. Nayar. Modeling the space of camera response functions. IEEE Transactions on Pattern Analysis and Machine Intelligence, 26(10):1272-1282, Oct 2004. 
Tom Haber, Christian Fuchs, Philippe Bekaert, Hans-Peter Seidel, Michael Goesele, and Hendrik P.A. Lensch. Relighting objects from image collections. In IEEE Conference on Computer Vision and Pattern Recognition, pages 627-634, 2009.

Yudeog Han, Joon-Young Lee, and In So Kweon. High quality shape from a single RGB-D image under uncalibrated natural illumination. In IEEE International Conference on Computer Vision, 2013.

S. Mohammadul Haque, Avishek Chatterjee, and Venu Madhav Govindu. High quality photometric reconstruction using a depth camera. In IEEE Conference on Computer Vision and Pattern Recognition, pages 2283-2290, 2014.

Kenji Hara, Ko Nishino, and Katsushi Ikeuchi. Light source position and reflectance estimation from a single view without the distant illumination assumption. IEEE Transactions on Pattern Analysis and Machine Intelligence, 27:493-505, 2005.

Keith Hartt and Mark Carlotto. A method for shape-from-shading using multiple images acquired under different viewing and lighting conditions. In IEEE Conference on Computer Vision and Pattern Recognition, 1989.

Daniel Hauagge, Scott Wehrwein, Kavita Bala, and Noah Snavely. Photometric ambient occlusion. In IEEE Conference on Computer Vision and Pattern Recognition, 2013.

Hideki Hayakawa. Photometric stereo under a light source with arbitrary motion. Journal of the Optical Society of America, 11(11):3079-3089, 1994.

Glenn Healey and Thomas O. Binford. Local shape from specularity. Technical report, Stanford University, 1986.

Carlos Hernandez, George Vogiatzis, and Roberto Cipolla. Multi-view photometric stereo. IEEE Transactions on Pattern Analysis and Machine Intelligence, 30:548-554, 2008.

Aaron Hertzmann and Steven M. Seitz. Shape and materials by example: a photometric stereo approach. In IEEE Conference on Computer Vision and Pattern Recognition, 2003.

Aaron Hertzmann and Steven M. Seitz. Example-based photometric stereo: shape reconstruction with general, varying BRDFs. IEEE Transactions on Pattern Analysis and Machine Intelligence, 27:1254-1264, 2005. ISSN 0162-8828.

Tomoaki Higo, Yasuyuki Matsushita, and Katsushi Ikeuchi. Consensus photometric stereo. In IEEE Conference on Computer Vision and Pattern Recognition, 2010. 
Michael Holroyd, Jason Lawrence, Greg Humphreys, and Todd E. Zickler. A photometric approach for estimating normals and tangents. ACM Transactions on Graphics, 27:133:1-133:9, 2008.

Berthold K. P. Horn. Shape from shading: A method for obtaining the shape of a smooth opaque object from one view. Technical report, MIT Artificial Intelligence Laboratory, 1970.

Berthold K. P. Horn and Michael J. Brooks. The variational approach to shape from shading. Computer Vision, Graphics, and Image Processing, 33:174-208, 1986.

Berthold K. P. Horn, Robert J. Woodham, and William M Silver. Determining shape and reflectance using multiple images. Technical report, MIT Artificial Intelligence Laboratory, 1978.

Itsik Horovitz and Nahum Kiryati. Depth from gradient fields and control points: Bias correction in photometric stereo. Image and Vision Computing, 22:681-694, 2004.

Chun-Ho Hung, Tai-Pang Wu, Y. Matsushita, Li Xu, Jiaya Jia, and ChiKeung Tang. Photometric stereo in the wild. In IEEE Winter Conference on Applications of Computer Vision (WACV), pages 302-309, 2015.

Satoshi Ikehata and Kiyoharu Aizawa. Photometric stereo using constrained bivariate regression for general isotropic surfaces. In IEEE Conference on Computer Vision and Pattern Recognition, pages 2187-2194, 2014.

Satoshi Ikehata, David Wipf, Yasuyuki Matsushita, and Kiyoharu Aizawa. Robust photometric stereo using sparse regression. In IEEE Conference on Computer Vision and Pattern Recognition, 2012.

Nathan Jacobs, Nathaniel Roman, and Robert Pless. Consistent temporal variations in many outdoor scenes. In IEEE Conference on Computer Vision and Pattern Recognition, 2007a.

Nathan Jacobs, Scott Satkin, Nathaniel Roman, Richard Speyer, and Robert Pless. Geolocating static cameras. In IEEE International Conference on Computer Vision, 2007b.

Nathan Jacobs, Brian Bies, and Robert Pless. Using cloud shadows to infer scene structure and camera calibration. In IEEE Conference on Computer Vision and Pattern Recognition, 2010.

Nathan Jacobs, Austin Abrams, and Robert Pless. Two cloud-based cues for estimating scene structure and camera calibration. IEEE Transactions on Pattern Analysis and Machine Intelligence, 10:2526-2538, 2013a. 
Nathan Jacobs, Mohammad T. Islam, and Scott Workman. Cloud motion as a calibration cue. In IEEE Conference on Computer Vision and Pattern Recognition, 2013b.

Hailin Jin, Stefano Soatto, and Anthony J. Yezzi. Multi-view stereo beyond lambert. In IEEE Conference on Computer Vision and Pattern Recognition, 2003.

Hailin Jin, D. Cremers, Anthony J. Yezzi, and S. Soatto. Shedding light on stereoscopic segmentation. In IEEE Conference on Computer Vision and Pattern Recognition, 2004.

Hailin Jin, Stefano Soatto, and Anthony J. Yezzi. Multi-view stereo reconstruction of dense shape and complex appearance. International Journal of Computer Vision, 63:175-189, 2005.

Micah K. Johnson and Edward H. Adelson. Shape estimation in natural illumination. In IEEE Conference on Computer Vision and Pattern Recognition, 2011.

Neel Joshi and David Kriegman. Shape from varying illumination and viewpoint. In IEEE Conference on Computer Vision and Pattern Recognition, 2007.

Jiyoung Jung, Joon-Young Lee, and In So Kweon. One-day outdoor photometric stereo via skylight estimation. In The IEEE Conference on Computer Vision and Pattern Recognition, June 2015.

Masayuki Kanbara and Naokazu Yokoya. Real-time estimation of light source environment for photorealistic augmented reality. In International Conference on Pattern Recognition, 2004.

Seon Joo Kim, Jan-Michael Frahm, and M. Pollefeys. Radiometric calibration with illumination change for outdoor scene analysis. In IEEE Conference on Computer Vision and Pattern Recognition, 2008.

Reinhard Klette and Karsten Schluens. Height data from gradient fields. In Machine Vision Applications, Architectures, and Systems IntegrationV, pages 204-215, 1996.

Sanjeev J. Koppal and Srinivasa G. Narasimhan. Clustering appearance for scene analysis. In IEEE Conference on Computer Vision and Pattern Recognition, pages 1323-1330, 2006.

Sanjeev J. Koppal and Srinivasa G. Narasimhan. Novel depth cues from uncalibrated near-field lighting. In IEEE International Conference on Computer Vision, pages 1-8, 2007. 
Kazutada Koshikawa. A polarimetric approach to shape understanding of glossy objects. In Proceedings of the 6th International Joint Conference on Artificial Intelligence - Volume 1, IJCAI '79, pages 493-495, San Francisco, CA, USA, 1979. Morgan Kaufmann Publishers Inc. ISBN 0-934613-47-8.

David J. Kriegman and Peter N. Belhumeur. What shadows reveal about object structure. Journal of the Optical Society of America, 18:1804-1813, 2001.

Pierre-Yves Laffont, Adrien Bousseau, Sylvain Paris, Fredo Durand, and George Drettakis. Coherent intrinsic images from photo collections. ACM Transactions on Graphics, 31:202:1-202:11, 2012.

Pierre-Yves Laffont, Adrien Bousseau, and George Drettakis. Rich intrinsic image decomposition of outdoor scenes from multiple views. IEEE Transactions on Visualization and Computer Graphics, 19:210-224, 2013.

Jean-Francois Lalonde, Srinivasa G. Narasimhan, and Alexei Efros. What does the sky tell us about the camera? In European Conference on Computer Vision, pages 354-367, 2008.

Jean-Francois Lalonde, Alexei Efros, and Srinivasa G. Narasimhan. Webcam clip art: Appearance and illuminant transfer from time-lapse sequences. ACM Transactions on Graphics, 28(5):131:1-131:10, December 2009.

Jean-Francois Lalonde, Srinivasa G. Narasimhan, and Alexei Efros. What do the sun and the sky tell us about the camera? International Journal of Computer Vision, 88:24-51, 2010.

Jason Lawrence, Aner Ben-Artzi, Christopher DeCoro, Wojciech Matusik, Hanspeter Pfister, Ravi Ramamoorthi, and Szymon M. Rusinkiewicz. Inverse shade trees for non-parametric material representation and editing. ACM Transactions on Graphics, 25:735-745, 2006.

Kyong Joon Lee, Qi Zhao, Xin Tong, Minmin Gong, Shahram Izadi, Sang Uk Lee, Ping Tan, and Stephen Lin. Estimation of intrinsic image sequences from image+depth video. In European Conference on Computer Vision, 2012.

Victor S. Lempitsky and Yuri Boykov. Global optimization for shape fitting. In IEEE Conference on Computer Vision and Pattern Recognition, 2007.

Hendrik P. A. Lensch, Jan Kautz, Michael Goesele, Wolfgang Heidrich, and Hans-Peter Seidel. Image-based reconstruction of spatial appearance and geometric detail. ACM Transactions on Graphics, 22:234-257, 2003.

Miao Liao, Liang Wang, Ruigang Yang, and Minglun Gong. Light fall-off stereo. In IEEE Conference on Computer Vision and Pattern Recognition, pages 1-8, 2007. 
Jongwoo Lim, J. Ho, Ming-Hsuan Yang, and D. Kriegman. Passive photometric stereo from motion. In IEEE International Conference on Computer Vision, 2005.

Stephen Lin, Jinwei Gu, Shuntaro Yamazaki, and Heung-Yeung Shum. Radiometric calibration from a single image. In IEEE Conference on Computer Vision and Pattern Recognition, 2004.

Stephen Lombardi and Ko Nishino. Reflectance and natural illumination from a single image. In European Conference on Computer Vision, 2012.

Feng Lu, Yasuyuki Matsushita, Imari Sato, Takahiro Okabe, and Yoichi Sato. Uncalibrated photometric stereo for unknown isotropic reflectances. In IEEE Conference on Computer Vision and Pattern Recognition, 2013.

Jiping Lu and Jim Little. Reflectance function estimation and shape recovery from image sequences of a rotating object. In IEEE International Conference on Computer Vision, 1995.

Sebastian Magda, David J. Kriegman, Todd E. Zickler, and Peter N. Belhumeur. Beyond lambert: Reconstructing surfaces with arbitrary BRDFs. In IEEE International Conference on Computer Vision, pages 391-398, 2001.

Satya P. Mallick, Todd E. Zickler, David J. Kriegman, and Peter N. Belhumeur. Beyond lambert: Reconstructing specular surfaces using color. In Proceedings of the IEEE Computer Society Conference on Computer Vision and Pattern Recognition, Volume 2, pages 619-626, Washington, DC, USA, 2005. IEEE Computer Society. ISBN 0-7695-2372-2.

Stephen R. Marschner. Inverse Rendering for Computer Graphics. PhD thesis, Cornell University, 1998.

Vincent Masselus, Philip Dutré, and Frederik Anrys. The free form light stage. In Eurographics Workshop on Rendering Techniques, 2002.

Wojciech Matusik, Hanspeter Pfister, Matt Brand, and Leonard McMillan. A data-driven reflectance model. ACM Transactions on Graphics, 22:759-769, 2003.

Roberto Mecca, Aaron Wetzler, Alfred M. Bruckstein, and Ron Kimmel. Near field photometric stereo with point light sources. SIAM Journal on Imaging Sciences, 7:2732-2770, 2014.

Steven H. Mersch. Polarized lighting for machine vision applications. In Proceedings of the Robotics International of SME 3rd Annual Applied Machine Vision Conference, pages 40-54, 1984. 
Srinivasa G. Narasimhan, Chi Wang, and Shree K. Nayar. All the images of an outdoor scene. In European Conference on Computer Vision, pages 148-162, 2002.

Shree K. Nayar, Katsushi Ikeuchi, and Takeo Kanade. Determining shape and reflectance of lambertian, specular, and hybrid surfaces using extended sources. In International Workshop on Industrial Applications of Machine Intelligence and Vision, 1989.

Shree K. Nayar, Katsushi Ikeuchi, and Takeo Kanade. Shape from interreflections. In IEEE International Conference on Computer Vision, 1990.

Shree K. Nayar, Xi-Sheng Fang, and Terrance Boult. Removal of specularities using color and polarization. In IEEE Conference on Computer Vision and Pattern Recognition, 1993.

Diego Nehab, Szymon M. Rusinkiewicz, James Davis, and Ravi Ramamoorthi. Efficiently combining positions and normals for precise 3D geometry. ACM Transactions on Graphics, 24:536-543, 2005.

Diego Nehab, Tim Weyrich, and Szymon M. Rusinkiewicz. Dense 3D reconstruction from specularity consistency. In IEEE Conference on Computer Vision and Pattern Recognition, 2008.

F. E. Nicodemus, J. C. Richmond, J. J. Hsia, I. W. Ginsberg, and T. Limperis. Geometrical Considerations and Nomenclature for Reflectance. U.S. Department of Commerce, 1977.

Takahiro Okabe, Imari Sato, and Yoichi Sato. Attached shadow coding: Estimating surface normals from shadows under unknown reflectance and lighting conditions. In IEEE International Conference on Computer Vision, 2009.

Takayuki Okatani and Koichiro Deguchi. On the Wiberg algorithm for matrix factorization in the presence of missing components. International Journal of Computer Vision, 2007.

Takayuki Okatani, Takahiro Yoshida, and Koichiro Deguchi. Efficient algorithm for low-rank matrix factorization with missing components and performance comparison of latest algorithms. In IEEE International Conference on Computer Vision, 2011.

Geoffrey Oxholm and Ko Nishino. Shape and reflectance from natural illumination. In European Conference on Computer Vision, 2012.

Geoffrey Oxholm and Ko Nishino. Multiview shape and reflectance from natural illumination. In IEEE Conference on Computer Vision and Pattern Recognition, pages 2163-2170, 2014. 
Alexandros Panagopoulos, Chaohui Wang, Dimitris Samaras, and Nikos Paragios. Illumination estimation and cast shadow detection through a higherorder graphical model. In IEEE Conference on Computer Vision and Pattern Recognition, 2011.

Thoma Papadhimitri and Paolo Favaro. A new perspective on uncalibrated photometric stereo. In IEEE Conference on Computer Vision and Pattern Recognition, 2013.

Jaesik Park, Sudipta N. Sinha, Yasuyuki Matsushita, Yu-Wing Tai, and In So Kweon. Multiview photometric stereo using planar mesh parameterization. In IEEE International Conference on Computer Vision, 2013.

James A. Paterson, David Claus, and Andrew W. Fitzgibbon. BRDF and geometry capture from extended inhomogeneous samples using flash photography. In Eurographics, 2005.

Alex Paul Pentland. A new sense for depth of field. IEEE Transactions on Pattern Analysis and Machine Intelligence, 4:523-531, 1987.

Richard Perez, Robert Seals, and Joseph Michalsky. All-weather model for sky luminance distribution-preliminary configuration and validation. Solar Energy, 50:235-245, 1993.

Bui Tuong Phong. Illumination for computer generated pictures. Communications of the ACM, 18:311-317, 1975.

Point Grey Research, Inc. Mono camera sensor review, 2015. http://www. ptgrey.com/support/downloads/10501.

Mark W. Powell, Sudeep Sarkar, and Dmitry B. Goldgof. A simple strategy for calibrating the geometry of light sources. IEEE Transactions on Pattern Analysis and Machine Intelligence, 2001.

Arcot J. Preetham, Peter Shirley, and Brian Smits. A practical analytic model for daylight. In Proceedings of the 26th Annual Conference on Computer Graphics and Interactive Techniques, pages 91-100, New York, NY, USA, 1999. ACM Press/Addison-Wesley Publishing Co. ISBN 0-201-48560-5.

Ravi Ramamoorthi and Pat Hanrahan. A signal-processing framework for inverse render. In Proceedings of ACM SIGGRAPH, 2001.

Rajarshi Ray, John Birk, and Robert B. Kelley. Error analysis of surface normals determined by radiometry. IEEE Transactions on Pattern Analysis and Machine Intelligence, 5:631-645, 1983.

Thomas C. Rindfleisch. A photometric method for deriving lunar topographic information. Technical report, California Insitute of Technology, 1965. 
Mark A. Robertson, Sean Borman, and Robert L. Stevenson. Dynamic range improvement through multiple exposures. In IEEE International Conference on Image Processing, pages 159-163, 1999.

Mark A. Robertson, Sean Borman, and Robert L. Stevenson. Estimationtheoretic approach to dynamic range enhancement using multiple exposures. Journal of Electronic Imaging, 12:219-228, 2003.

Fabiano Romeiro and Todd E. Zickler. Blind reflectometry. In European Conference on Computer Vision, 2010a.

Fabiano Romeiro and Todd E. Zickler. Inferring reflectance under real-world illumination. Technical report, Harvard University, 2010b.

Roland Ruiters and Reinhard Klein. Heightfield and spatially varying BRDF reconstruction for materials with interreflections. Computer Graphics Forum, 28:513-522, 2009.

Szymon M. Rusinkiewicz. A new change of variables for efficient BRDF representation. In Rendering Techniques '98, pages 11-22. Springer, 1998.

Imari Sato, Yoichi Sato, and Katsushi Ikeuchi. Acquiring a radiance distribution to superimpose virtual objects onto a real scene. Transactions on Visualization and Computer Graphics, 1999.

Imari Sato, Takahiro Okabe, Qiong Yu, and Yoichi Sato. Shape reconstruction based on similarity in radiance changes under varying illumination. In IEEE International Conference on Computer Vision, pages 1-8, 2007.

Yoichi Sato and Katsushi Ikeuchi. Reflectance analysis under solar illumination. Technical report, Carnegie Mellon University, 1994a.

Yoichi Sato and Katsushi Ikeuchi. Temporal-color space analysis of reflection. Journal of the Optical Society of America, 11, 1994b.

Yoichi Sato and Katsushi Ikeuchi. Reflectance analysis under solar illumination. In Proceedings of the Workshop on Physics-Based Modeling in Computer Vision, pages 180-187, 1995.

Volker Schuster. BRDF based photo-consistency. Diploma thesis, Computer Graphics department, University of Bonn, 2010.

Steve M. Seitz, Brian Curless, James Diebel, Daniel Scharstein, and Richard Szeliski. A comparison and evaluation of multi-view stereo reconstruction algorithms. In IEEE Conference on Computer Vision and Pattern Recognition, pages 519-526, 2006.

Steven A. Shafer. Using color to separate reflection components. Technical report, University of Rochester, 1984. 
Qi Shan, Riley Adams, Brian Curless, Yasutaka Furukawa, and Steven M. Seitz. The visual Turing test for scene reconstruction. In International Conference on 3D Vision, 2013.

L. Shen and P. Tan. Photometric stereo and weather estimation using internet images. In IEEE Conference on Computer Vision and Pattern Recognition, 2009 .

Boxin Shi, Yasuyuki Matsushita, Yichen Wei, Chao Xu, and Ping Tan. Selfcalibrating photometric stereo. In IEEE Conference on Computer Vision and Pattern Recognition, 2010.

Boxin Shi, Ping Tan, Yasuyuki Matsushita, and Katsushi Ikeuchi. Elevation angle from reflectance monotonicity: Photometric stereo for general isotropic reflectances. In European Conference on Computer Vision, 2012a.

Boxin Shi, Ping Tan, Yasuyuki Matsushita, and Katsushi Ikeuchi. A biquadratic reflectance model for radiometric image analysis. In IEEE Conference on Computer Vision and Pattern Recognition, $2012 \mathrm{~b}$.

William Michael Silver. Determining shape and reflectance using multiple images. Master's thesis, Massachusetts Institute of Technology, 1980.

Noah Snavely, Steven M. Seitz, and Richard Szeliski. Photo tourism: exploring photo collections in 3D. ACM Transactions on Graphics, 25(3):835-846, 2006.

Stefano Soatto, Anthony J. Yezzi, and Hailin Jin. Tales of shape and radiance in multi-view stereo. In IEEE International Conference on Computer Vision, 2003.

Kalyan Sunkavalli, Wojciech Matusik, Hanspeter Pfister, and Szymon M. Rusinkiewicz. Factored time-lapse video. ACM Transactions on Graphics, 2007.

Kalyan Sunkavalli, Fabiano Romeiro, Wojciech Matusik, Todd E. Zickler, and Hanspeter Pfister. What do color changes reveal about an outdoor scene? In IEEE Conference on Computer Vision and Pattern Recognition, 2008.

Kalyan Sunkavalli, Todd E. Zickler, and Hanspeter Pfister. Visibility subspaces: Uncalibrated photometric stereo with shadows. In European Conference on Computer Vision, 2010.

Hemant D. Tagare and Rui J. P. deFigueiredo. A theory of photometric stereo for a class of diffuse non-lambertian surfaces. IEEE Transactions on Pattern Analysis and Machine Intelligence, 13, 1991.

Ping Tan and Todd E. Zickler. A projective framework for radiometric image analysis. In IEEE Conference on Computer Vision and Pattern Recognition, 2009. 
Ping Tan, Satya P. Mallick, Long Quan, David J. Kriegman, and Todd E. Zickler. Isotropy, reciprocity and the generalized bas-relief ambiguity. In IEEE Conference on Computer Vision and Pattern Recognition, 2007.

Ping Tan, Long Quan, and Todd E. Zickler. The geometry of reflectance symmetries. IEEE Transactions on Pattern Analysis and Machine Intelligence, 33, 2011.

Robby T. Tan and Katsushi Ikeuchi. Separating reflection components of textured surfaces using a single image. IEEE Transactions on Pattern Analysis and Machine Intelligence, 27:178-193, 2005.

Marco Tarini, Hendrik P. A. Lensch, Michael Goesele, and Hans-Peter Seidel. 3D acquisition of mirroring objects using striped patterns. Graphical Models, 67:233-259, 2005.

Joshua B. Tenenbaum, Vin de Silva, and John C. Langford. A global geometric framework for nonlinear dimensionality reduction. Science, 290:2319-2323, 2000 .

William Thompson, Roland Fleming, Sarah Creem-Regehr, and Jeanine Kelly Stefanucci. Visual Perception from a Computer Graphics Perspective. A. K. Peters, Ltd., Natick, MA, USA, 1st edition, 2011. ISBN 1568814658, 9781568814650 .

James Tompkin, Kwang In Kim, Jan Kautz, and Christian Theobalt. Videoscapes: Exploring sparse, unstructured video collections. ACM Transactions on Graphics, 2012.

Kenneth E. Torrance and Ephraim M. Sparrow. Theory for off-specular reflection from roughened surfaces. Journal of the Optical Society of America, 57:1105-1112, 1967.

Adrien Treuille, Aaron Hertzmann, and Steven M. Seitz. Example-based stereo with general BRDFs. In European Conference on Computer Vision, 2004.

Borom Tunwattanapong, Graham Fyffe, Paul Graham, Jay Busch, Xueming $\mathrm{Yu}$, Abhijeet Ghosh, and Paul Debevec. Acquiring reflectance and shape from continuous spherical harmonic illumination. ACM Transactions on Graphics, 2013.

Frank Verbiest and Luc Van Gool. Photometric stereo with coherent outlier handling and confidence estimation. In IEEE Conference on Computer Vision and Pattern Recognition, 2008.

Daniel Vlasic, Pieter Peers, Ilya Baran, Paul Debevec, Jovan Popovic, Szymon M. Rusinkiewicz, and Wojciech Matusik. Dynamic shape capture using multi-view photometric stereo. ACM Transactions on Graphics, 28, 2009. 
George Vogiatzis, Carlos Hernandez, and Roberto Cipolla. Reconstruction in the round using photometric normals and silhouettes. In IEEE Conference on Computer Vision and Pattern Recognition, 2006.

Yang Wang and Dimitris Samaras. Estimation of multiple illuminants from a single image of arbitrary known geometry. In European Conference on Computer Vision, 2002.

Gregory J. Ward. Measuring and modeling anisotropic reflection. In Proceedings of ACM SIGGRAPH, pages 265-272, 1992.

Martin Weber and Roberto Cipolla. A practical method for estimation of point light-sources. In British Machine Vision Conference, 2001.

Martin Weber, Andrew Blake, and Roberto Cipolla. Towards a complete dense geometric and photometric reconstruction under varying pose and illumination. In British Machine Vision Conference, 2002.

Michael Weinmann, Roland Ruiters, Aljosa Osep, Christopher Schwartz, and Reinhard Klein. Fusing structured light consistency and Helmholtz normals for 3D reconstruction. In British Machine Vision Conference, pages 1-12, 2012.

Michael Weinmann, Aljosa Osep, Roland Ruiters, and Reinhard Klein. Multiview normal field integration for 3D reconstruction of mirroring objects. In IEEE International Conference on Computer Vision, pages 2504-2511, 2013.

Holger Winnemoeller, Ankit Mohan, Jack Tumblin, and Bruce Gooch. Light waving: Estimating light positions from photographs alone. Computer Graphics Forum, 2005.

Kwan-Yee Kenneth Wong, Dirk Schnieders, and Shuda Li. Recovering light directions and camera poses from a single sphere. In European Conference on Computer Vision, 2008.

Robert J. Woodham. Reflectance Map Techniques for Analyzing Surface Defects in Metal Castings. PhD thesis, Massachusetts Institute of Technology, 1977.

Robert J. Woodham. Photometric method for determining surface orientation from multiple images. Optical Engineering, pages 139-144, 1980.

Chenglei Wu, Yebin Liu, Qionghai Dai, and Bennett Wilburn. Fusing multiview and photometric stereo for 3D reconstruction under uncalibrated illumination. IEEE Transactions on Visualization and Computer Graphics, 17, 2011. 
Chenglei Wu, Kiran Varanasi, and Christian Theobalt. Full body performance capture under uncontrolled and varying illumination: A shading-based approach. In European Conference on Computer Vision, 2012.

Lun Wu, Arvind Ganesh, Boxin Shi, Yasuyuki Matsushita, Yongtian Wang, and Yi Ma. Robust photometric stereo via low-rank matrix completion and recovery. In 12th Asian Conference on Computer Vision, 2010.

Tai-Pang Wu and Chi-Keung Tang. Dense photometric stereo by expectation maximization. In European Conference on Computer Vision, 2006.

Zhe Wu and Ping Tan. Calibrating photometric stereo by holistic reflectance symmetry analysis. In IEEE Conference on Computer Vision and Pattern Recognition, 2013.

Zhongquan $\mathrm{Wu}$ and Lingxiao Li. A line-integration based method for depth recovery from surface normals. Computer Vision, Graphics, and Image Processing, 43:53-66, 1988.

Wuyuan Xie, Yunbo Zhang, Charlie C.L. Wang, and Ronald C.-K. Chung. Surface-from-gradients: An approach based on discrete geometry processing. In IEEE Conference on Computer Vision and Pattern Recognition, pages 2203-2210, 2014.

Ying Xiong, Kate Saenko, Trevor Darrell, and Todd E. Zickler. From pixels to physics: Probabilistic color de-rendering. In IEEE Conference on Computer Vision and Pattern Recognition, 2012.

S. Xu and A.M. Wallace. Recovering surface reflectance and multiple light locations and intensities from image data. Pattern Recognition Letters, 2008.

Ruigang Yang, Marc Pollefeys, and Greg Welch. Dealing with textureless regions and specular highlights - a progressive space carving scheme using a novel photo-consistency measure. In IEEE International Conference on Computer Vision, 2003.

Kuk-Jin Yoon, Emmanuel Prados, and Peter Sturm. Joint estimation of shape and reflectance using multiple images with known illumination conditions. International Journal of Computer Vision, 86:192-210, 2010.

Yusuke Yoshiyasu and Nobutoshi Yamazaki. Topology-adaptive multi-view photometric stereo. In IEEE Conference on Computer Vision and Pattern Recognition, 2011.

Lap-Fai Yu, Sai-Kit Yeung, Yu-Wing Tai, Demetri Terzopoulos, and Tony F. Chan. Outdoor photometric stereo. In IEEE International Conference on Computational Photography, 2013. 
Tianli $\mathrm{Yu}$, Ning $\mathrm{Xu}$, and Narendra Ahuja. Shape and view independent reflectance map from multiple views. In European Conference on Computer Vision, 2004.

Alan Yuille and Daniel Snow. Shape and albedo from multiple images using integrability. In IEEE Conference on Computer Vision and Pattern Recognition, 1997.

Li Zhang, Brian Curless, Aaron Hertzmann, and Steven M. Seitz. Shape and motion under varying illumination: Unifying structure from motion, photometric stereo, and multiview stereo. In IEEE International Conference on Computer Vision, pages 618-625, 2003.

Ruo Zhang, Ping-Sing Tsai, James Edwin Cryer, and Mubarak Shah. Shape from shading: A survey. IEEE Transactions on Pattern Analysis and Machine Intelligence, 21, 1999.

S. Kevin Zhou, Gaurav Aggarwal, Rama Chellappa, and David W. Jacobs. Appearance characterization of linear lambertian objects, generalized photometric stereo and illumination-invariant face recognition. IEEE Transactions on Pattern Analysis and Machine Intelligence, 29, 2007.

Wei Zhou and Chandra Kambhamettu. Estimation of illuminant direction and intensity of multiple light sources. In European Conference on Computer Vision, 2002.

Wei Zhou and Chandra Kambhamettu. A unified framework for scene illuminant estimation. In British Machine Vision Conference, 2004.

Zhenglong Zhou, Zhe Wu, and Ping Tan. Multi-view photometric stereo with spatially varying isotropic materials. In IEEE Conference on Computer Vision and Pattern Recognition, 2013.

Todd E. Zickler. Reciprocal image features for uncalibrated Helmholtz stereopsis. In IEEE Conference on Computer Vision and Pattern Recognition, 2006.

Todd E. Zickler, Peter N. Belhumeur, and David J. Kriegman. Helmholtz stereopsis: Exploiting reciprocity for surface reconstruction. International Journal of Computer Vision, 49, 2002. 